\title{
EXISTENCE FOR EVOLUTIONARY NEUMANN PROBLEMS WITH LINEAR GROWTH BY STABILITY RESULTS
}

\author{
Leah Schätzler \\ Universität Erlangen-Nürnberg, Department Mathematik \\ Cauerstrasse 11, 91058 Erlangen, Germany; schaetzler@math.fau.de
}

\begin{abstract}
We are concerned with the Neumann type boundary value problem to parabolic systems

$$
\partial_{t} u-\operatorname{div}\left(D_{\xi} f(x, D u)\right)=-D_{u} g(x, u)
$$

where $u$ is vector-valued, $f$ satisfies a linear growth condition and $\xi \mapsto f(x, \xi)$ is convex. We prove that variational solutions of such systems can be approximated by variational solutions to

$$
\partial_{t} u-\operatorname{div}\left(D_{\xi} f^{p}(x, D u)\right)=-D_{u} g(x, u)
$$

with $p>1$. This can be interpreted both as a stability and existence result for general flows with linear growth.
\end{abstract}

\section{Introduction and results}

Throuhgout this paper, we fix dimensions $n, N \in \mathbf{N}$, a bounded Lipschitz domain $\Omega \subset \mathbf{R}^{n}$ and a finite time interval $(0, T)$ with $0<T<\infty$. By $\Omega_{T}$ we denote the space-time cylinder $\Omega \times(0, T)$, by $S_{T}$ the lateral boundary $\partial \Omega \times(0, T)$ and by $\partial_{\text {par }} \Omega$ the parabolic boundary $S_{T} \cup(\Omega \times\{0\})$. We are concerned with the $L^{2}$-gradient flow associated with variational functionals of the type

$$
\mathbf{I}[u]=\int_{\Omega}[f(x, D u)+g(x, u)] \mathrm{d} x
$$

with Borel measurable functions $f: \Omega \times \mathbf{R}^{N \times n} \rightarrow[0, \infty)$ and $g: \Omega \times \mathbf{R}^{N} \rightarrow[0, \infty)$, for vector-valued maps $u: \Omega_{T} \rightarrow \mathbf{R}^{N}$. We assume that $f$ satisfies the linear growth condition

$$
\nu|\xi| \leq f(x, \xi) \leq L(1+|\xi|) \quad \text { for all }(x, \xi) \in \Omega \times \mathbf{R}^{N \times n}
$$

with structure constants $0<\nu \leq L$ and that

$$
\xi \mapsto f(x, \xi) \text { is convex. }
$$

In addition, we suppose that the partial map $u \mapsto g(x, u)$ is convex. On a formal level, solutions of this gradient flow correspond to solutions of the system of parabolic partial differential equations with a natural boundary condition

$$
\begin{cases}\partial_{t} u-\operatorname{div}\left(D_{\xi} f(x, D u)\right)=-D_{u} g(x, u) & \text { in } \Omega_{T}, \\ \sum_{j=1}^{n} \frac{\partial f}{\partial \xi_{j}^{2}}(x, D u) \mathbf{n}_{j}=0 & \text { on } S_{T}, i=1, \ldots, N, \\ u(x, 0)=u_{o}(x) & \text { on } \Omega \times\{0\},\end{cases}
$$

https://doi.org/10.5186/aasfm.2019.4461

2010 Mathematics Subject Classification: Primary 35K87, 35B35, 49J40, $49 J 45$.

Key words: Total variation flow, flows with linear growth, stability, solutions obtained as limits of approximations. 
where $\mathbf{n}$ denotes the outer unit normal of $\Omega$ and $\xi=\left(\xi_{j}^{i}\right)_{1 \leq i \leq N, 1 \leq j \leq n}$. In the case of the model integrand $f(x, \xi)=|\xi|$ this boundary condition reads as $\sum_{j=1}^{n}|D u|^{-1} D u_{j}^{i} \mathbf{n}_{j}=$ 0 on the lateral boundary, which implies the Neumann boundary condition $\frac{\partial u}{\partial \mathbf{n}}=0$. Inspired by Lichnewsky and Temam [28], we consider variational solutions to (1.3). For the precise notion of solution cf. Definition 1.1. In the present paper, solutions to (1.3) are constructed by approximating $f$ by $f^{p}$ with $p>1$ and then letting $p \downarrow 1$. Since $f^{p}$ satisfies a standard $p$-growth and coercivity condition for $p>1$, there exists a very well developed existence theory for solutions of the $L^{2}$-gradient flow associated with

$$
\mathbf{I}_{p}[u]=\int_{\Omega}\left[f^{p}(x, D u)+g(x, u)\right] \mathrm{d} x,
$$

which formally corresponds to the system of partial differential equations with a natural boundary condition

$$
\begin{cases}\partial_{t} u-\operatorname{div}\left(D_{\xi} f^{p}(x, D u)\right)=-D_{u} g(x, u) & \text { in } \Omega_{T}, \\ \sum_{j=1}^{n} \frac{\partial f^{p}}{\partial \xi_{j}^{l}}(x, D u) \mathbf{n}_{j}=0 & \text { on } S_{T}, i=1, \ldots, N, \\ u(x, 0)=u_{o}(x) & \text { on } \Omega \times\{0\} .\end{cases}
$$

The construction of solutions to (1.3) as limits of solutions to (1.4) can also be interpreted as a stability result in the limit $p \downarrow 1$. Note that we have not approximated $g$, since it would not simplify the existence result for the gradient flow associated with $\mathbf{I}_{p}$. However it could be the aim of a future work to investigate stability for more general integrands $f_{i}$ and $g_{i}$ converging to $f$, respectively $g$ in a suitable sense.

At this point, an overview over related existence and stability results is given. A well investigated special case is the total variation flow, which formally corresponds to

$$
\partial_{t} u-\operatorname{div}\left(\frac{D u}{|D u|}\right)=0 \quad \text { in } \Omega_{T} .
$$

Different notions of solutions such as entropy solutions (allowing $L^{1}$-initial data), weak solutions and variational solutions and different boundary conditions have been considered in the existence results $[2,3,4,5,6,7,11,17,23]$. The obstacle problem associated with the total variation flow has been treated in [16]. Gradient flows related to functionals consisting of the total variation plus certain lower order terms are applied in image restoration. Existence of variational solutions to such flows has been shown in [13] by elliptic regularization both for Cauchy-Dirichlet and Neumann boundary values. The existence of variational solutions to the time dependent minimal surface problem has been established by Lichnewsky and Temam, cf. [28]. Spadaro and Stefanelli proved the existence of solutions to the time dependent minimal surface problem and more general continuous integrands of linear growth in [32] by elliptic regularization (also called weighted energy dissipation). Here, for a.e. $t \in(0, T)$, a solution satisfies the equation $u^{\prime}(t)+\partial \mathcal{F}(u(t)) \ni 0$, where $\partial \mathcal{F}$ denotes the subdifferential of the relaxation $\mathcal{F}$ of the given integral functional $\mathbf{F}$. Solutions to more general equations of the type

$$
\partial_{t} u-\operatorname{div}(\mathbf{a}(x, D u))=0 \quad \text { in } \Omega_{T},
$$

where $\mathbf{a}(x, \xi)=D_{\xi} f(x, \xi)$ for an integrand $f: \Omega \times \mathbf{R}^{n} \rightarrow \mathbf{R}$, have been constructed by Andreu, Caselles and Mazón, cf. $[5,8,9]$. Here, the integrand $f$ satisfies a linear growth condition. For any fixed $x$ the partial map $\xi \mapsto f(x, \xi)$ is assumed to be convex and differentiable. Furthermore, a continuity condition similar to (1.8) is imposed on the recession function $f^{\infty}$, allowing the application of a variant of Reshetnyak's 
continuity theorem. Observe that the differentiability assumption on the partial map $\xi \mapsto f(x, \xi)$ excludes integrands like $f(\xi)=|\xi|$ or $f(x, \xi)=a(x)|\xi|$.

While there is a wide range of existence results for functionals with linear growth, only a few stability results are known. Although the focus is not on stability but on existence, stability methods were used in [18], where de León and Webler were concerned with the inhomogeneous total variation flow with zero Dirichlet boundary values. More precisely, the intermediate existence result for more regular data given in [18, Sec. 4] relies on approximation by solutions of the inhomogeneous parabolic $p$-Laplacian. This is slightly related to [33], where Tölle showed that solutions to the inhomogeneous total variation flow can be approximated by solutions to the inhomogeneous parabolic $p$-Laplacian if the initial data and the inhomogenities converge in a suitable sense. Here, the case of zero Dirichlet boundary values as well as Neumann boundary values is considered. Another stability result concerning $p \downarrow 1$ has been obtained by Gianazza and Klaus in [22]. For a bounded $C^{1}$-domain and time independent Dirichlet boundary values the authors constructed solutions of the total variation flow as limit of solutions of the parabolic $p$-Laplacian. The overall proof strategy in the present paper is the same as in [14]. The authors use stability methods to prove the existence of variational solutions to Cauchy-Dirichlet problems of the type

$$
\begin{cases}\partial_{t} u-\operatorname{div}\left(D_{\xi} f(x, D u)\right)=0 & \text { in } \Omega_{T} \\ u=g & \text { on } \partial_{\mathrm{par}} \Omega\end{cases}
$$

where $f$ satisfies a linear growth and coercivity condition and is convex with respect to the gradient variable and the boundary values $g$ are possibly time dependent.

In contrast, stability in the case $p \rightarrow p_{o}>1$ is well established. For a recent result see [21]. Here, Fujishima, Habermann, Kinnunen and Masson showed that parabolic $Q$-quasiminimizers related to the parabolic $p$-Laplace equation with given boundary values are stable with respect to parameters $Q$ and $p$. Since the proof relies on higher integrability of the gradient, the result is restricted to the case $p>\frac{2 n}{n+2}$. In the degenerate case $p \geq 2$, a stability result for solutions of parabolic $p$-Laplace equations has previously been obtained by Kinnunen and Parviainen, cf. [25].

1.1. Convex functionals with linear growth. Note that $f$ is a Carathéodory function, since (1.1) and (1.2) together imply that $\xi \mapsto f(x, \xi)$ is Lipschitz continuous (cf. Lemma 2.10). Assumption (1.1) ensures that

$$
\mathbf{F}[u]:=\int_{\Omega} f(x, D u) \mathrm{d} x
$$

is finite on the Sobolev space $W^{1,1}\left(\Omega, \mathbf{R}^{N}\right)$. However under the above conditions (and even under reasonable extra assumptions) the infimum of $\mathbf{F}$ will not be attained in $W^{1,1}\left(\Omega, \mathbf{R}^{N}\right)$, but only in $\operatorname{BV}\left(\Omega, \mathbf{R}^{N}\right)$. As usual, $\operatorname{BV}\left(\Omega, \mathbf{R}^{N}\right)$ denotes the space of functions $u \in L^{1}\left(\Omega, \mathbf{R}^{N}\right)$ with finite total variation

$$
|D u|(\Omega):=\sup \left\{\int_{\Omega} u \cdot \operatorname{div} \varphi \mathrm{d} x: \varphi \in C_{0}^{1}\left(\Omega, \mathbf{R}^{N \times n}\right),\|\varphi\|_{L^{\infty}\left(\Omega, \mathbf{R}^{N}\right)} \leq 1\right\}<\infty .
$$

Note that $D u \in \operatorname{RM}\left(\Omega, \mathbf{R}^{N \times n}\right)$, the space of finite vector-valued Radon measures. The norm in $\operatorname{BV}\left(\Omega, \mathbf{R}^{N}\right)$ is defined by

$$
\|u\|_{\mathrm{BV}\left(\Omega, \mathbf{R}^{N}\right)}:=\|u\|_{L^{1}\left(\Omega, \mathbf{R}^{N}\right)}+|D u|(\Omega) .
$$

Therefore we need to extend $\mathbf{F}$ from $W^{1,1}\left(\Omega, \mathbf{R}^{N}\right)$ to $\operatorname{BV}\left(\Omega, \mathbf{R}^{N}\right)$. To this end we define the so called recession function $f^{\infty}$ of $f$, which takes into account the jumps 
of BV functions. More precisely, for an integrand satisfying (1.1) we set

$$
f^{\infty}(x, \xi):=\liminf _{\substack{\tilde{x} \rightarrow x, \tilde{\xi} \rightarrow \xi \\ t \downarrow 0}} t f\left(\tilde{x}, t^{-1} \tilde{\xi}\right) \quad \text { for }(x, \xi) \in \bar{\Omega} \times\left(\mathbf{R}^{N \times n} \backslash\{0\}\right),
$$

and $f^{\infty}(x, 0)=0$ for all $x \in \bar{\Omega}$. Note that $f^{\infty}$ is positively 1-homogeneous and convex in $\xi$. Moreover, $f^{\infty}$ fulfills the following linear growth and coercivity condition

$$
\nu|\xi| \leq f^{\infty}(x, \xi) \leq L|\xi| \text { for all }(x, \xi) \in \bar{\Omega} \times \mathbf{R}^{N \times n} .
$$

In addition to the assumptions on $f$ above, we impose the continuity condition

$$
\lim _{\substack{\tilde{x} \rightarrow x, \tilde{\xi} \rightarrow \xi \\ t \downarrow 0}} t f\left(\tilde{x}, t^{-1} \tilde{\xi}\right) \quad \text { exists in } \mathbf{R}
$$

for every $(x, \xi) \in \bar{\Omega} \times\left(\mathbf{R}^{N \times n} \backslash\{0\}\right)$, which ensures that the lower limit in definition (1.6) is indeed a limit and that the recession function $f^{\infty}$ is continuous with respect to $(x, \xi)$ on $\bar{\Omega} \times \mathbf{R}^{N \times n}$. Let $u$ be a function of bounded variation. By $D^{a} u$ we denote the absolutely continuous part and by $D^{s} u$ the singular part of the Lebesgue decomposition of $D u$ with respect to the Lebesgue measure $\mathcal{L}^{n}$. Moreover, $\nabla u$ denotes the Radon-Nikodym density of $D^{a} u$ with respect to $\mathcal{L}^{n}$, i.e. we have

$$
D u=D^{a} u+D^{s} u=\nabla u \mathcal{L}^{n}+D^{s} u .
$$

Using the preceding definitions, we extend the functional $\mathbf{F}: W^{1,1}\left(\Omega, \mathbf{R}^{N}\right) \rightarrow[0, \infty)$ to a convex functional $\mathcal{F}: \operatorname{BV}\left(\Omega, \mathbf{R}^{N}\right) \rightarrow[0, \infty)$ by

$$
\mathcal{F}[u]:=\int_{\Omega} f(x, \nabla u) \mathrm{d} x+\int_{\Omega} f^{\infty}\left(x, \frac{D^{s} u}{\left|D^{s} u\right|}\right) \mathrm{d}\left|D^{s} u\right|
$$

for any $u \in \operatorname{BV}\left(\Omega, \mathbf{R}^{N}\right)$. Note that $\mathbf{F}=\mathcal{F}$ on $W^{1,1}\left(\Omega, \mathbf{R}^{N}\right)$.

1.2. Variational solutions. Furthermore, we assume that the Borel measurable function $g: \Omega \times \mathbf{R}^{N} \rightarrow[0, \infty)$ satisfies the growth condition

$$
0 \leq g(x, u) \leq M\left(1+|u|^{2}\right) \quad \text { for all }(x, \xi) \in \Omega \times \mathbf{R}^{N}
$$

with a structure constant $M>0$ and that

$$
u \mapsto g(x, u) \quad \text { is convex. }
$$

Note that the preceding two conditions together imply that $u \mapsto g(x, u)$ is locally Lipschitz continuous, cf. Lemma 2.10. To obtain a rigorous formulation of the problem associated with $f$, we consider the parabolic function space $L_{w *}^{1}\left(0, T ; \operatorname{BV}\left(\Omega, \mathbf{R}^{N}\right)\right)$ consisting of the weakly* measurable maps $u:(0, T) \rightarrow \operatorname{BV}\left(\Omega, \mathbf{R}^{N}\right)$ that fulfill $t \mapsto$ $\|u(t)\|_{\mathrm{BV}\left(\Omega, \mathbf{R}^{N}\right)} \in L^{1}(0, T)$; cf. Section 2.1 for a precise definition. A Cauchy datum is given by

$$
u_{o} \in L^{2}\left(\Omega, \mathbf{R}^{N}\right)
$$

In the present paper we study variational solutions to the Cauchy-Neumann problem associated with (1.3) in the sense of the following definition.

Definition 1.1. (Variational solution for functionals with linear growth) Assume that the Cauchy datum $u_{o}$ fulfills (1.12), that $f$ satisfies hypotheses (1.1), (1.2) and (1.8), that the functional $\mathcal{F}$ is given as in (1.9) and that $g$ fulfills (1.10) and (1.11). We identify a measurable map $u: \Omega_{T} \rightarrow \mathbf{R}^{N}$ in the class

$$
u \in L^{\infty}\left(0, T ; L^{2}\left(\Omega, \mathbf{R}^{N}\right)\right) \cap L_{w *}^{1}\left(0, T ; \operatorname{BV}\left(\Omega, \mathbf{R}^{N}\right)\right)
$$


as a variational solution to the gradient flow associated with (1.3) if and only if the variational inequality

$$
\begin{aligned}
& \int_{0}^{\tau} \mathcal{F}[u] \mathrm{d} t+\iint_{\Omega_{\tau}} g(x, u) \mathrm{d} x \mathrm{~d} t \\
& \leq \iint_{\Omega_{\tau}} \partial_{t} v \cdot(v-u) \mathrm{d} x \mathrm{~d} t+\int_{0}^{\tau} \mathcal{F}[v] \mathrm{d} t+\iint_{\Omega_{\tau}} g(x, v) \mathrm{d} x \mathrm{~d} t \\
& \quad-\frac{1}{2}\|(v-u)(\tau)\|_{L^{2}\left(\Omega, \mathbf{R}^{N}\right)}^{2}+\frac{1}{2}\left\|v(0)-u_{o}\right\|_{L^{2}\left(\Omega, \mathbf{R}^{N}\right)}^{2}
\end{aligned}
$$

holds true for a.e. $\tau \in[0, T]$ and any comparison map $v \in L_{w *}^{1}\left(0, T ; \operatorname{BV}\left(\Omega, \mathbf{R}^{N}\right)\right)$ with $\partial_{t} v \in L^{1}\left(0, T ; L^{2}\left(\Omega, \mathbf{R}^{N}\right)\right)$ and $v(0) \in L^{2}\left(\Omega, \mathbf{R}^{N}\right)$.

Remark 1.2. Variational inequality (1.13) allows us to conclude that any variational solution in the sense of the preceding definition attains the initial datum in the $L^{2}$-sense; cf. Lemma 2.11 for details.

Next we are concerned with the approximations $f^{p}$ of $f$ with $p>1$. Note that for any $p>1$ the integrand $f^{p}: \Omega \times \mathbf{R}^{N \times n} \rightarrow \mathbf{R}$ satisfies the standard $p$-growth condition

$$
\nu^{p}|\xi|^{p} \leq f^{p}(x, \xi) \leq L^{p}(1+|\xi|)^{p} \leq L_{p}\left(1+|\xi|^{p}\right)
$$

for all $x \in \Omega$ and $\xi \in \mathbf{R}^{N \times n}$, where $L_{p}:=2^{p-1} L^{p}$. Since $f$ is non-negative and $(\cdot)^{p}$ is non-decreasing and convex on $\mathbf{R}_{0}^{+}$, we have that

$$
\xi \mapsto f^{p}(x, \xi) \text { is convex. }
$$

We consider Cauchy data

$$
u_{p, o} \in L^{2}\left(\Omega, \mathbf{R}^{N}\right)
$$

Definition 1.3. (Variational solution for functionals with superlinear growth) Let $u_{p, o}$ fulfill (1.16). Assume that $f^{p}$ satisfies hypotheses (1.14) and (1.15) and that $g$ satisfies (1.10) and (1.11). We identify a measurable map $u: \Omega_{T} \rightarrow \mathbf{R}^{N}$ in the class

$$
u \in C^{0}\left([0, T] ; L^{2}\left(\Omega, \mathbf{R}^{N}\right)\right) \cap L^{p}\left(0, T ; W^{1, p}\left(\Omega, \mathbf{R}^{N}\right)\right)
$$

as a variational solution to the gradient flow associated to (1.4) if the variational inequality

$$
\begin{aligned}
& \iint_{\Omega_{\tau}}\left[f^{p}(x, D u)+g(x, u)\right] \mathrm{d} x \mathrm{~d} t \\
& \leq \iint_{\Omega_{\tau}} \partial_{t} v \cdot(v-u) \mathrm{d} x \mathrm{~d} t+\iint_{\Omega_{\tau}}\left[f^{p}(x, D v)+g(x, v)\right] \mathrm{d} x \mathrm{~d} t \\
& \quad-\frac{1}{2}\|(v-u)(\tau)\|_{L^{2}\left(\Omega, \mathbf{R}^{N}\right)}^{2}+\frac{1}{2}\left\|v(0)-u_{p, o}\right\|_{L^{2}\left(\Omega, \mathbf{R}^{N}\right)}^{2}
\end{aligned}
$$

holds true for any $\tau \in[0, T]$ and any comparison map $v \in L^{p}\left(0, T ; W^{1, p}\left(\Omega, \mathbf{R}^{N}\right)\right)$ with $\partial_{t} v \in L^{1}\left(0, T ; L^{2}\left(\Omega, \mathbf{R}^{N}\right)\right)$ and $v(0) \in L^{2}\left(\Omega, \mathbf{R}^{N}\right)$.

Remark 1.4. Note that variational inequality (1.17) implies that any variational solution in the sense of the preceding definition attains the initial values $u_{p, o}$ in the sense that $u(t) \rightarrow u_{p, o}$ in $C^{0}\left([0, T] ; L^{2}\left(\Omega, \mathbf{R}^{N}\right)\right)$ as $t \downarrow 0$; see Lemma 2.14 below. Furthermore, if $u_{p, o} \in L^{2}\left(\Omega, \mathbf{R}^{N}\right) \cap W^{1, p}\left(\Omega, \mathbf{R}^{N}\right)$, $u$ possesses a time derivative $\partial_{t} u \in$ $L^{2}\left(\Omega_{T}, \mathbf{R}^{N}\right)$, cf. Theorem 3.1.

The following theorem is the main result of the present paper. 
Theorem 1.5. Assume that $f$ satisfies hypotheses (1.1), (1.2) and (1.8) and $g$ fulfills hypotheses (1.10) and (1.11). Let $p_{i}>1$ for $i \in \mathbf{N}$ with $p_{i} \downarrow 1$ as $i \rightarrow \infty$. Assume that the Cauchy data $u_{i, o}:=u_{p_{i}, o}$ satisfy assumption (1.16) and that

$$
u_{i, o} \rightarrow u_{o} \quad \text { in } L^{2}\left(\Omega, \mathbf{R}^{N}\right)
$$

For any $i \in \mathbf{N}$ let

$$
u_{i} \in C^{0}\left([0, T] ; L^{2}\left(\Omega, \mathbf{R}^{N}\right)\right) \cap L^{p_{i}}\left(0, T ; W^{1, p_{i}}\left(\Omega, \mathbf{R}^{N}\right)\right)
$$

be the variational solution to (1.4) in the sense of Definition 1.3 with Cauchy datum $u_{i, o}$. Then, there exists a limit map

$$
u \in C^{0}\left([0, T] ; L^{2}\left(\Omega, \mathbf{R}^{N}\right)\right) \cap L_{w *}^{1}\left(0, T ; \operatorname{BV}\left(\Omega, \mathbf{R}^{N}\right)\right)
$$

such that

$$
\begin{cases}u_{i} \rightarrow u & \text { in } L^{1}\left(\Omega_{T}, \mathbf{R}^{N}\right) \\ D u_{i} \stackrel{*}{\rightarrow} D u & \text { weakly* in } \operatorname{RM}\left(\Omega, \mathbf{R}^{N \times n}\right), \\ u_{i} \stackrel{*}{\rightarrow} u & \text { weakly* in } L^{\infty}\left(0, T ; L^{2}\left(\Omega, \mathbf{R}^{N}\right)\right)\end{cases}
$$

as $k \rightarrow \infty$. The limit function $u$ is the unique variational solution to (1.3) in the sense of Definition 1.1.

1.3. Plan of the paper. In Section 2, we collect results that we will need in the following sections. These are already known or their proofs are basically the same as in the case of Dirichlet boundary values. In particular, we prove a certain continuity property with respect to time in Section 2.7. This allows us to deduce compactness for the sequence of variational solutions associated with exponents $p>$ 1 , which is crucial for passing to the limit $p \downarrow 1$. However, we postponed wellknown results about continuity, uniqueness and localization of variational solutions in the case $p>1$ to the appendices $\mathrm{A}$ and $\mathrm{B}$, since we give longer proofs for the convenience of the reader. In addition, Appendix A contains the continuity and uniqueness results for $p=1$. Next, we establish the existence of variational solutions to problems with superlinear growth in the case of regular initial data in Section 3. This result is extended to the case of $L^{2}$-initial data in Section 4 . Note that in contrast to previous existence results, cf. for example [13], comparison functions with time derivative in $L^{1}\left(0, T ; L^{2}\left(\Omega, \mathbf{R}^{N}\right)\right)$ instead of $L^{2}\left(\Omega_{T}, \mathbf{R}^{N}\right)$ are admissible. However, this condition is more natural, since $L^{1}\left(0, T ; L^{2}\left(\Omega, \mathbf{R}^{N}\right)\right)$ is the predual of the space $L^{\infty}\left(0, T ; L^{2}\left(\Omega, \mathbf{R}^{N}\right)\right)$, in which the variational solutions are contained. In Section 5 we give the proof of the main result. More precisely, we first derive the convergence of the variational solutions associated with $p>1$ to a suitable limit map as $p \downarrow 1$. Using a version of Reshetnyak's lower semicontinuity theorem, Theorem 2.3, we establish the variational inequality for the limit map and certain regular comparison maps. In particular, we require $v \in L^{2}\left(0, T ; W^{1,2}\left(\Omega, \mathbf{R}^{N}\right)\right)$. By an approximation argument involving a version of Reshetnyak's continuity theorem, cf. Theorem 2.4, we finally prove the desired variational inequality for general comparison maps. Compared to the case of Dirichlet boundary values treated in [14], througout the present paper the construction of admissible comparison maps is somewhat easier, since we do not have to care about boundary values attained by mollifications. 


\section{Preliminaries}

A vital ingredient in the proof of Theorem 1.5 is the following compactness lemma. We use the abbreviation

$$
\tau_{h} f(t):=f(t+h)-f(t)
$$

for $h>0$ and $t \in(0, T-h)$.

Lemma 2.1. [31, Theorem 5] Let $X \subset B \subset Y$ be three Banach spaces such that the embedding $X \hookrightarrow B$ is compact and $1 \leq p<\infty$. Assume that the set $\mathfrak{F} \subset L^{p}(0, T ; X)$ is bounded and satisfies the property

$$
\left\|\tau_{h} f\right\|_{L^{p}(0, T-h ; Y)} \rightarrow 0 \quad \text { as } h \downarrow 0 \quad \text { uniformly for } f \in \mathfrak{F} .
$$

Then, the set $\mathfrak{F}$ is relatively compact in $L^{p}(0, T ; B)$.

2.1. Parabolic function spaces. Let $X$ be a Banach space and $1 \leq p \leq \infty$. By $L^{p}(0, T ; X)$ we denote the space of Bochner measurable functions $u:[0, T] \rightarrow X$ with $t \mapsto\|u(t)\|_{X} \in L^{p}(0, T)$. However, since $\operatorname{BV}\left(\Omega, \mathbf{R}^{N}\right)$ is not separable, assuming Bochner measurability is too restrictive in the case $X=\mathrm{BV}\left(\Omega, \mathbf{R}^{N}\right)$. For example the cone in space-time $u(t)=\chi_{B_{t}\left(x_{o}\right)}$ is not Bochner measurable, because its image is not separable in $\operatorname{BV}\left(\Omega, \mathbf{R}^{N}\right)$. Therefore, we consider weakly*-measurable functions instead. Observe that $\operatorname{BV}\left(\Omega, \mathbf{R}^{N}\right)$ is the dual of the separable Banach space $X_{0}$ consisting of the functions $g-\operatorname{div} G$ with $g \in C_{0}^{0}\left(\Omega, \mathbf{R}^{N}\right)$ and $G \in C_{0}^{0}\left(\Omega, \mathbf{R}^{N \times n}\right)$, see e.g. [1, Remark 3.12]. Hence, a function $v: I \rightarrow \mathrm{BV}\left(\Omega, \mathbf{R}^{N}\right)=X_{0}^{\prime}$ is called weakly*_ measurable if for every $\varphi \in X_{0}$ the mapping $I \ni t \mapsto\langle v(t), \varphi\rangle \in \mathbf{R}$ is measurable. Here $\langle\cdot, \cdot\rangle$ denotes the duality pairing between $\operatorname{BV}\left(\Omega, \mathbf{R}^{N}\right)$ and $X_{0}$. This definition implies that the norms $\|v(t)\|_{\mathrm{BV}\left(\Omega, \mathbf{R}^{N}\right)}$ depend measurably on $t \in I$. Indeed, the norm on $\operatorname{BV}\left(\Omega, \mathbf{R}^{N}\right)=X_{0}^{\prime}$ can be written as

$$
\|v(t)\|_{\mathrm{BV}\left(\Omega, \mathbf{R}^{N}\right)}=\sup \left\{\langle v(t), \varphi\rangle: \varphi \in X_{0},\|\varphi\|_{X_{0}} \leq 1\right\} .
$$

Since by definition $t \mapsto\langle v(t), \varphi\rangle$ is measurable and $X_{0}$ is separable, the supremum in the preceding equality depends measurably on $t \in I$. At this stage, we are able to define the parabolic function spaces

$$
\begin{aligned}
& L_{w *}^{p}\left(0, T ; \operatorname{BV}\left(\Omega, \mathbf{R}^{N}\right)\right) \\
& :=\left\{\begin{array}{l|l}
v:[0, T] \rightarrow \operatorname{BV}\left(\Omega, \mathbf{R}^{N}\right) \mid \begin{array}{l}
v \text { is weakly*-measurable with } \\
t \mapsto\|v(t)\|_{\mathrm{BV}\left(\Omega, \mathbf{R}^{N}\right)} \in L^{p}(0, T)
\end{array}
\end{array}\right\}
\end{aligned}
$$

for any $1 \leq p \leq \infty$. For a brief summary on the different notions of measurability and the related concepts of integration, cf. [19, Chap. 2].

We will need the following compactness property of the space $L_{w *}^{1}(0, T ; \mathrm{BV}(\Omega$, $\left.\mathbf{R}^{N}\right)$ ). Its proof is formulated in [16, Lemma 2.4] for the case $N=1$, but all of the arguments stay valid in the vector-valued case.

Lemma 2.2. Assume that the sequence $u_{i} \in L_{w *}^{1}\left(0, T ; \mathrm{BV}\left(\Omega, \mathbf{R}^{N}\right)\right)$ satisfies $u_{i} \rightarrow u$ weakly in $L^{1}\left(\Omega_{T}, \mathbf{R}^{N}\right)$ as $i \rightarrow \infty$ for some $u \in L^{1}\left(\Omega_{T}, \mathbf{R}^{N}\right)$ and

$$
\liminf _{i \rightarrow \infty} \int_{0}^{T}\left|D u_{i}(t)\right|(\Omega) \mathrm{d} t<\infty .
$$

Then we have $u \in L_{w *}^{1}\left(0, T ; \operatorname{BV}\left(\Omega, \mathbf{R}^{N}\right)\right)$ and

$$
\int_{0}^{T}|D u(t)|(\Omega) \mathrm{d} t \leq \liminf _{i \rightarrow \infty} \int_{0}^{T}\left|D u_{i}(t)\right|(\Omega) \mathrm{d} t .
$$


2.2. Reshetnyak (semi-)continuity. From [30, Corollary 4.15] we infer the following result on lower semicontinuity of the functional $\mathcal{F}$. For related results cf. also [1], [20] and [26, Theorem 2].

Theorem 2.3. (Reshetnyak's lower semicontinuity theorem) Assume that $\Omega \subset$ $\mathbf{R}^{n}$ is a bounded Lipschitz domain and that $f: \Omega \times \mathbf{R}^{N \times n} \rightarrow[0, \infty)$ is a Carathéodory function satisfying (1.1) and (1.8) and that $\xi \mapsto f(x, \xi)$ is quasiconvex for every $x \in \Omega$. Further, suppose that the functional $\mathcal{F}$ is given as in (1.9). For functions $u_{k}, u \in \operatorname{BV}\left(\Omega, \mathbf{R}^{N}\right)$ with $u_{k} \rightarrow u$ in $L^{1}\left(\Omega, \mathbf{R}^{N}\right)$ as $k \rightarrow \infty$ we have that

$$
\mathcal{F}[u] \leq \liminf _{k \rightarrow \infty} \mathcal{F}\left[u_{k}\right]
$$

Proof. We assume that the right-hand side of (2.1) is finite, since otherwise, the claim trivially holds. Hence, we can find a subsequence $u_{k_{j}}$ with

$$
\nu \limsup _{j \rightarrow \infty}\left|D u_{k_{j}}\right|(\Omega) \leq \lim _{j \rightarrow \infty} \mathcal{F}\left[u_{k_{j}}\right]=\liminf _{k \rightarrow \infty} \mathcal{F}\left[u_{k}\right]<\infty
$$

Since $u_{k_{j}} \rightarrow u$ in $L^{1}(\Omega)$ by assumption, we deduce that $u_{k_{j}} \stackrel{*}{\rightarrow} u$ weakly* in $\operatorname{BV}\left(\Omega, \mathbf{R}^{N}\right)$ as $j \rightarrow \infty$. Therefore, we are in a position to apply [30, Corollary 4.15], which yields

$$
\mathcal{F}[u] \leq \lim _{j \rightarrow \infty} \mathcal{F}\left[u_{k_{j}}\right]=\liminf _{k \rightarrow \infty} \mathcal{F}\left[u_{k}\right],
$$

which is assertion (2.1).

Next, we state the following version of Reshetnyak's continuity theorem which is obtained by specializing [10, Theorem 3.10] to bounded domains; see also [27, Theorem 3] for a related result.

Theorem 2.4. (Reshetnyak's continuity theorem) Suppose that $\Omega$ is bounded, that $\partial \Omega$ has zero $\mathcal{L}^{n}$-measure and that $f: \Omega \times \mathbf{R}^{m} \rightarrow \mathbf{R}$ fulfills (1.1), (1.2) and (1.8). Moreover assume that $\left(\mu_{k}\right)_{k \in \mathbf{N}}$ weak ${ }^{*}$-converges to $\mu$ in the space of finite $\mathbf{R}^{m}$-valued Radon measures on $\bar{\Omega}$. If there holds

$$
\lim _{k \rightarrow \infty}\left|\left(\mathcal{L}^{n}, \mu_{k}\right)\right|(\bar{\Omega})=\left|\left(\mathcal{L}^{n}, \mu\right)\right|(\bar{\Omega})
$$

for the $\mathbf{R}^{m+1}$-valued measures $\left(\mathcal{L}^{n}, \mu_{k}\right)$ and $\left(\mathcal{L}^{n}, \mu\right)$, then we have

$$
\begin{aligned}
& \lim _{k \rightarrow \infty}\left[\int_{\Omega} f\left(\cdot, \frac{\mathrm{d} \mu_{k}^{a}}{\mathrm{~d} \mathcal{L}^{n}}\right) \mathrm{d} x+\int_{\bar{\Omega}} f^{\infty}\left(\cdot, \frac{\mathrm{d} \mu_{k}^{s}}{\mathrm{~d}\left|\mu_{k}^{s}\right|}\right) \mathrm{d}\left|\mu_{k}^{s}\right|\right] \\
& =\int_{\Omega} f\left(\cdot, \frac{\mathrm{d} \mu^{a}}{\mathrm{~d} \mathcal{L}^{n}}\right) \mathrm{d} x+\int_{\bar{\Omega}} f^{\infty}\left(\cdot, \frac{\mathrm{d} \mu^{s}}{\mathrm{~d}\left|\mu^{s}\right|}\right) \mathrm{d}\left|\mu^{s}\right| .
\end{aligned}
$$

In the preceding theorem $\left(\mathcal{L}^{n}, \mu\right)(A)$ is nothing else than $\left(\mathcal{L}^{n}(A), \mu(A)\right)$. Moreover, $\left|\left(\mathcal{L}^{n}, \mu\right)\right|(\bar{\Omega})$ denotes the total variation of the vector-valued measure $\left(\mathcal{L}^{n}, \mu\right)$. In terms of the Lebesgue decomposition $\mu=\mu^{a}+\mu^{s}=\frac{d \mu^{a}}{d \mathcal{L}^{n}} \mathcal{L}^{n}+\mu^{s}$ the total variation can be expressed as follows

$$
\left|\left(\mathcal{L}^{n}, \mu\right)\right|(\bar{\Omega})=\int_{\Omega} \sqrt{1+\left|\frac{d \mu^{a}}{d \mathcal{L}^{n}}\right|^{2}} \mathrm{~d} x+\left|\mu^{s}\right|(\bar{\Omega}) .
$$

2.3. Mollification in space. In order to prove Theorem 1.5, we need to regularize certain comparison maps. To this end we first extend these functions from $\Omega$ to $\mathbf{R}^{n}$ by means of the following lemma. Its proof can be found in [17, Lemma 2.3] for the case $N=1$ and $\partial_{t} v \in L^{2}\left(\Omega_{T}\right)$. However, the arguments extend to the case considered in the present paper. 
Lemma 2.5. Assume that $\Omega \subset \mathbf{R}^{n}$ is a domain with bounded Lipschitz boundary. Then there exists a bounded linear extension operator $E: \operatorname{BV}\left(\Omega, \mathbf{R}^{N}\right) \rightarrow \operatorname{BV}\left(\mathbf{R}^{n}\right.$, $\mathbf{R}^{N}$ ) with the properties

(i) $|D E v|(\partial \Omega)=0$ for every $v \in \operatorname{BV}\left(\Omega, \mathbf{R}^{N}\right)$,

(ii) if $v \in L^{2}\left(\Omega, \mathbf{R}^{N}\right) \cap \operatorname{BV}\left(\Omega, \mathbf{R}^{N}\right)$, then $E v \in L^{2}\left(\mathbf{R}^{n}, \mathbf{R}^{N}\right) \cap \mathrm{BV}\left(\mathbf{R}^{n}, \mathbf{R}^{N}\right)$,

(iii) if $v \in L^{2}\left(\Omega_{T}, \mathbf{R}^{N}\right) \cap L_{w *}^{1}\left(0, T ; \operatorname{BV}\left(\Omega, \mathbf{R}^{N}\right)\right)$, then $E v \in L^{2}\left(\mathbf{R}^{n} \times(0, T), \mathbf{R}^{N}\right) \cap$ $L_{w *}^{1}\left(0, T ; \operatorname{BV}\left(\mathbf{R}^{n}, \mathbf{R}^{N}\right)\right)$. If $\partial_{t} v \in L^{1}\left(0, T ; L^{2}\left(\Omega, \mathbf{R}^{N}\right)\right)$ holds true, then also $\partial_{t} E v \in L^{1}\left(0, T ; L^{2}\left(\mathbf{R}^{n}, \mathbf{R}^{N}\right)\right)$.

Let $\phi_{\varepsilon}$ denote the standard mollifier on $\mathbf{R}^{n}$. We compile properties of the spatial regularization $v_{\varepsilon}:=v * \phi_{\varepsilon}$ of a map $v$ in the following lemma.

Lemma 2.6. Let $v \in L_{w *}^{1}\left(0, T ; B V\left(\mathbf{R}^{n}, \mathbf{R}^{N}\right)\right)$ with $\partial_{t} v \in L^{1}\left(0, T ; L^{2}\left(\mathbf{R}^{n}, \mathbf{R}^{N}\right)\right)$ and $v(0) \in L^{2}\left(\mathbf{R}^{n}, \mathbf{R}^{N}\right)$. Then the following assertions hold true:

(i) $v_{\varepsilon} \rightarrow v$ in $L^{2}\left(\mathbf{R}^{n} \times(0, T) ; \mathbf{R}^{N}\right)$ as $\varepsilon \downarrow 0$,

(ii) $v_{\varepsilon} \in C^{0}\left([0, T] ; W^{1,2}\left(\Omega, \mathbf{R}^{N}\right)\right)$ for any $\varepsilon>0$,

(iii) for any $\varepsilon>0$ we have

$$
\sup _{t \in[0, T]}\left\|v_{\varepsilon}(t)\right\|_{L^{2}\left(\mathbf{R}^{n}, \mathbf{R}^{N}\right)} \leq \sup _{t \in[0, T]}\|v(t)\|_{L^{2}\left(\mathbf{R}^{n}, \mathbf{R}^{N}\right)},
$$

(iv) $\partial_{t} v_{\varepsilon} \rightarrow \partial_{t} v$ in $L^{1}\left(0, T ; L^{2}\left(\Omega, \mathbf{R}^{N}\right)\right)$ as $\varepsilon \downarrow 0$

(v) in the limit $\varepsilon \downarrow 0$ we have for a.e. $t \in[0, T]$ that

$$
\left\{\begin{array}{l}
D v_{\varepsilon}(t) \stackrel{*}{\rightarrow} D v(t) \quad \text { weakly* in } \operatorname{RM}\left(\mathbf{R}^{n}, \mathbf{R}^{N \times n}\right), \\
\left|\left(\mathcal{L}^{n}, D v_{\varepsilon}(t)\right)\right|(\bar{\Omega}) \rightarrow\left|\left(\mathcal{L}^{n}, D v(t)\right)\right|(\bar{\Omega}) .
\end{array}\right.
$$

Proof. Assertions (i), (iii) and (iv) are standard properties of mollifications. Next, we prove (ii). Note that $v_{\varepsilon}(0) \in C^{\infty}\left(\mathbf{R}^{n}, \mathbf{R}^{N}\right)$. Hence $v_{\varepsilon}(0) \in W^{1,2}\left(\Omega, \mathbf{R}^{N}\right)$ holds true as well. Since $\phi_{\varepsilon} \in C_{0}^{\infty}\left(\mathbf{R}^{n}, \mathbf{R}^{N}\right)$ is independent of time and $\partial_{t} v \in$ $L^{1}\left(0, T ; L^{2}\left(\mathbf{R}^{n}, \mathbf{R}^{N}\right)\right)$, we have that

$$
\partial_{t} v_{\varepsilon}=\left(\partial_{t} v\right) * \phi_{\varepsilon}
$$

that

$$
\partial_{t} D v_{\varepsilon}(x, t)=\int_{\mathbf{R}^{n}} \partial_{t} v(y, t) \otimes D_{x} \phi_{\varepsilon}(x-y) \mathrm{d} y
$$

and that $\partial_{t} v_{\varepsilon}, \partial_{t} D v_{\varepsilon} \in L^{1}\left(0, T ; L^{2}\left(\Omega, \mathbf{R}^{N}\right)\right)$. Indeed, by Young's inequality for convolutions we obtain

$$
\begin{aligned}
\left\|\partial_{t} v_{\varepsilon}\right\|_{L^{1}\left(0, T ; L^{2}\left(\Omega, \mathbf{R}^{N}\right)\right)} & \leq \int_{0}^{T}\left\|\phi_{\varepsilon}\right\|_{L^{1}\left(\mathbf{R}^{n}\right)}\left\|\partial_{t} v\right\|_{L^{2}\left(\mathbf{R}^{n}, \mathbf{R}^{N}\right)} \mathrm{d} t \\
& =\left\|\phi_{\varepsilon}\right\|_{L^{1}\left(\mathbf{R}^{n}\right)}\left\|\partial_{t} v\right\|_{L^{1}\left(0, T ; L^{2}\left(\mathbf{R}^{n}, \mathbf{R}^{N}\right)\right)} \\
& =\left\|\partial_{t} v\right\|_{L^{1}\left(0, T ; L^{2}\left(\mathbf{R}^{n}, \mathbf{R}^{N}\right)\right)}
\end{aligned}
$$

and

$$
\begin{aligned}
\left\|\partial_{t} D v_{\varepsilon}\right\|_{L^{1}\left(0, T ; L^{2}\left(\Omega, \mathbf{R}^{N}\right)\right)} & \leq \int_{0}^{T}\left\|D \phi_{\varepsilon}\right\|_{L^{1}\left(\mathbf{R}^{n}, \mathbf{R}^{n}\right)}\left\|\partial_{t} v\right\|_{L^{2}\left(\mathbf{R}^{n}, \mathbf{R}^{N}\right)} \mathrm{d} t \\
& =\left\|D \phi_{\varepsilon}\right\|_{L^{1}\left(\mathbf{R}^{n}, \mathbf{R}^{n}\right)}\left\|\partial_{t} v\right\|_{L^{1}\left(0, T ; L^{2}\left(\Omega, \mathbf{R}^{N}\right)\right)} \\
& =c(n, \varepsilon)\left\|\partial_{t} v\right\|_{L^{1}\left(0, T ; L^{2}\left(\Omega, \mathbf{R}^{N}\right)\right)}
\end{aligned}
$$

This yields claim (ii). Finally, we are concerned with claim (v). Observe that

$$
\sup _{\varepsilon \in(0,1)}\left|D v_{\varepsilon}(t)\right|\left(\mathbf{R}^{n}\right) \leq|D v(t)|\left(\mathbf{R}^{n}\right) \quad \text { for a.e. } t \in[0, T] \text {. }
$$


Since $v_{\varepsilon}(t) \rightarrow v(t)$ in $L^{2}\left(\mathbf{R}^{n}, \mathbf{R}^{N}\right)$ for a.e. $t \in[0, T]$, the limit of any weakly* convergent subsequence of $D v_{\varepsilon}(t)$ is $D v(t)$, which proves the first part of $(v)$. Then also $\left(\mathcal{L}^{n}, D v_{\varepsilon}(t)\right) \rightarrow\left(\mathcal{L}^{n}, D v(t)\right)$ weakly* in $\operatorname{RM}\left(\bar{\Omega}, \mathbf{R}^{N n+1}\right)$. By lower semicontinuity, this implies

$$
\left|\left(\mathcal{L}^{n}, D v(t)\right)\right|(\bar{\Omega}) \leq \liminf _{\varepsilon \downarrow 0}\left|\left(\mathcal{L}^{n}, D v_{\varepsilon}(t)\right)\right|(\bar{\Omega}) .
$$

Moreover, we compute that for a.e. $t \in[0, T]$ and all $\varepsilon>0$

$$
\left|\left(\mathcal{L}^{n}, D v_{\varepsilon}(t)\right)\right|(\bar{\Omega})=\left|(1, D v(t)) * \phi_{\varepsilon} \mathcal{L}^{n}\right|(\bar{\Omega}) \leq\left|\left(\mathcal{L}^{n}, D v(t)\right)\right|(\bar{\Omega}) .
$$

This yields assertion (v).

2.4. Mollification in time. Let $h \in(0, T], X$ be a separable Banach space and $v_{o} \in X$. We consider $v \in L^{r}(0, T ; X)$ for some $1 \leq r \leq \infty$ and define for any $t \in[0, T]$ the mollification in time by

$$
\llbracket v \rrbracket_{h}(t):=\mathrm{e}^{-\frac{t}{h}} v_{o}+\frac{1}{h} \int_{0}^{t} \mathrm{e}^{\frac{s-t}{h}} v(s) \mathrm{d} s .
$$

Sometimes we will also use the notation $\llbracket v \rrbracket_{h}^{v_{o}}$ to make clear which initial datum is used. By construction, this regularization formally solves the ordinary differential equation

$$
\partial_{t} \llbracket v \rrbracket_{h}=-\frac{1}{h}\left(\llbracket v \rrbracket_{h}-v\right)
$$

with initial condition $\llbracket v \rrbracket_{h}(0)=v_{o}$. The following lemma states some basic properties of the mollifications $\llbracket \cdot \rrbracket_{h}$, cf. [12, Appendix B] or [24, Lemma 2.2] for the proofs of the statements.

Lemma 2.7. Suppose that $X$ is a separable Banach space and $v_{o} \in X$. If $v \in L^{r}(0, T ; X)$ for some $r \geq 1$, then the mollification $\llbracket v \rrbracket_{h}$ defined in (2.2) fulfills $\llbracket v \rrbracket_{h} \in L^{r}(0, T ; X)$ and for any $t_{o} \in(0, T]$ there holds

$$
\left\|\llbracket v \rrbracket_{h}\right\|_{L^{r}\left(0, t_{o} ; X\right)} \leq\|v\|_{L^{r}\left(0, t_{o} ; X\right)}+\left[\frac{h}{r}\left(1-\mathrm{e}^{-\frac{t_{o} r}{h}}\right)\right]^{\frac{1}{r}}\left\|v_{o}\right\|_{X} .
$$

In the case $r=\infty$ the bracket $[\ldots]^{\frac{1}{r}}$ in the preceding inequality has to be interpreted as 1 . Moreover, in the case $r<\infty$ we have $\llbracket v \rrbracket_{h} \rightarrow v$ in $L^{r}(0, T ; X)$ as $h \downarrow 0$. Finally, if $v \in C^{0}([0, T] ; X)$ and $v_{o}=v(0)$, then $\llbracket v \rrbracket_{h} \in C^{0}([0, T] ; X),[v]_{h}(0)=v_{o}$, and moreover $\llbracket v \rrbracket_{h} \rightarrow v$ in $C^{0}([0, T] ; X)$ as $h \downarrow 0$.

For maps $v \in L^{r}(0, T ; X)$ with $\partial_{t} v \in L^{r}(0, T ; X)$ we have the following assertion.

Lemma 2.8. Let $X$ be a separable Banach space and $r \geq 1$. Assume that $v \in L^{r}(0, T ; X)$ with $\partial_{t} v \in L^{r}(0, T ; X)$. Then, for the mollification in time defined by

$$
\llbracket v \rrbracket_{h}(t):=\mathrm{e}^{-\frac{t}{h}} v(0)+\frac{1}{h} \int_{0}^{t} \mathrm{e}^{\frac{s-t}{h}} v(s) \mathrm{d} s
$$

the time derivative can be computed by

$$
\partial_{t} \llbracket v \rrbracket_{h}(t)=\frac{1}{h} \int_{0}^{t} \mathrm{e}^{\frac{s-t}{h}} \partial_{s} v(s) \mathrm{d} s
$$

and, moreover we have that

$$
\left\|\partial_{t} \llbracket v \rrbracket_{h}\right\|_{L^{r}(0, T ; X)} \leq\left\|\partial_{t} v\right\|_{L^{r}(0, T ; X)}
$$

holds true. 
Moreover, for a proof of the following lemma for the case $N=1 \mathrm{cf}$. [13, Lemma 2.6]. Note that the proof can easily be extended to the vectorial case.

Lemma 2.9. Let $v_{o} \in \operatorname{BV}\left(\Omega, \mathbf{R}^{N}\right)$ and $v \in L_{w *}^{1}\left(0, T ; \operatorname{BV}\left(\Omega, \mathbf{R}^{N}\right)\right)$. Then, the time mollification according to $(2.2)$ with initial values $v_{o}$ satisfies $\llbracket v \rrbracket_{h} \in L_{w *}^{1}(0, T$; $\left.\mathrm{BV}\left(\Omega, \mathbf{R}^{N}\right)\right)$ and $\left|D \llbracket v \rrbracket_{h}(t)\right|(\Omega) \leq \llbracket|D v|(\Omega) \rrbracket_{h}(t)$ holds true for a.e. $t \in[0, T]$, where $\llbracket|D v|(\Omega) \rrbracket_{h}$ is the time mollification according to $(2.2)$ with initial values $\left|D v_{o}\right|(\Omega)$.

2.5. Lipschitz continuity. Here we state a local Lipschitz continuity assertion that we apply for the integrands $f^{p}, p \geq 1$ with $m=N n$ and for $g$ with $m=N$ and $p=p_{o}=2$; for the proof cf. [14, Lemma 2.11]. See also [1, Lemma 5.42] and [29] for related results. Note that the following Lemma ensures in particular that the Lipschitz constant does not blow up as $p \downarrow 1$.

Lemma 2.10. Let $p_{o} \geq 1$ and assume that $h(x, \cdot): \mathbf{R}^{m} \rightarrow \mathbf{R}$ is convex for a.e. $x \in \Omega$ and satisfies

$$
|h(x, \xi)| \leq L\left(1+|\xi|^{p}\right) \quad \text { for a.e. } x \in \Omega \text { and all } \xi \in \mathbf{R}^{m}
$$

for constants $p \in\left[1, p_{o}\right]$ and $L>0$. Then $h$ fulfills the local Lipschitz continuity condition

$$
|h(x, \xi)-h(x, \eta)| \leq c\left(m, p_{o}\right) L\left(1+|\xi|^{p-1}+|\eta|^{p-1}\right)|\xi-\eta|
$$

for a.e. $x \in \Omega$ and all $\eta, \xi \in \mathbf{R}^{m}$.

2.6. The initial condition. Here, we show that variational solutions to (1.3) and (1.4) attain the initial datum $u_{o}$ respectively $u_{p, o}$.

Lemma 2.11. Assume that $u_{o}$ is as in (1.12), that $f$ satisfies hypotheses (1.1), (1.2) and (1.8) and that $g$ fulfills (1.10) and (1.11). Then any variational solution $u$ in the sense of Definition 1.1 attains the initial datum $u_{o}$ in the $L^{2}$-sense, i.e.

$$
\lim _{h \downarrow 0} \frac{1}{h} \int_{0}^{h}\left\|u(t)-u_{o}\right\|_{L^{2}\left(\Omega, \mathbf{R}^{N}\right)}^{2} \mathrm{~d} t=0 .
$$

Proof. Let $h>0$. We extend $u_{o}$ to $\mathbf{R}^{n}$ by zero and consider the mollification $u_{o, \sqrt{h}}:=u_{o} * \phi_{\sqrt{h}}$, where $\phi_{\sqrt{h}}$ denotes the standard mollifier in $\mathbf{R}^{n}$. Note that $v \equiv u_{o, \sqrt{h}}$ is admissible in (1.13). Using also the growth conditions (1.1) and (1.10), we infer that

$$
\begin{aligned}
& \frac{1}{4}\left\|u(\tau)-u_{o}\right\|_{L^{2}\left(\Omega, \mathbf{R}^{N}\right)}^{2} \leq \frac{1}{2}\left\|u(\tau)-u_{o, \sqrt{h}}\right\|_{L^{2}\left(\Omega, \mathbf{R}^{N}\right)}^{2}+\frac{1}{2}\left\|u_{o}-u_{o, \sqrt{h}}\right\|_{L^{2}\left(\Omega, \mathbf{R}^{N}\right)}^{2} \\
& \leq \tau L \int_{\Omega}\left(1+\left|D u_{o, \sqrt{h}}\right|\right) \mathrm{d} x+\tau M \int_{\Omega}\left(1+\left|u_{o, \sqrt{h}}\right|^{2}\right) \mathrm{d} x+\left\|u_{o}-u_{o, \sqrt{h}}\right\|_{L^{2}\left(\Omega, \mathbf{R}^{N}\right)}^{2}
\end{aligned}
$$

We estimate the first term on the right-hand side of the preceding inequality by Young's inequality for convolutions and Hölder's inequality. This yields

$$
\begin{aligned}
\left\|D u_{o, \sqrt{h}}\right\|_{L^{1}\left(\Omega, \mathbf{R}^{N}\right)} & \leq\left\|u_{o}\right\|_{L^{1}\left(\Omega, \mathbf{R}^{N}\right)}\left\|D \phi_{\sqrt{h}}\right\|_{L^{1}\left(\mathbf{R}^{n}\right)} \\
& \leq|\Omega|^{\frac{1}{2}}\left\|u_{o}\right\|_{L^{2}\left(\Omega, \mathbf{R}^{N}\right)} \frac{1}{\sqrt{h}}\|D \phi\|_{L^{1}\left(\mathbf{R}^{n}\right)} \\
& =c(n,|\Omega|) \frac{1}{\sqrt{h}}\left\|u_{o}\right\|_{L^{2}\left(\Omega, \mathbf{R}^{N}\right)} .
\end{aligned}
$$


Inserting this into the second last inequality, integrating over $\tau \in(0, h)$ and dividing by $h$, we have that

$$
\begin{aligned}
\frac{1}{4 h} \int_{0}^{h}\left\|u(\tau)-u_{o}\right\|_{L^{2}\left(\Omega, \mathbf{R}^{N}\right)}^{2} \mathrm{~d} \tau \leq & \frac{h}{2}(L+M)|\Omega|+c(n,|\Omega|) L \sqrt{h}\left\|u_{o}\right\|_{L^{2}\left(\Omega, \mathbf{R}^{N}\right)} \\
& +\frac{h}{2} M\left\|u_{o, \sqrt{h}}\right\|_{L^{2}\left(\Omega, \mathbf{R}^{N}\right)}^{2}+\left\|u_{o}-u_{o, \sqrt{h}}\right\|_{L^{2}\left(\Omega, \mathbf{R}^{N}\right)}^{2} \\
& \rightarrow 0
\end{aligned}
$$

in the limit $h \downarrow 0$, since $u_{o, \sqrt{h}} \rightarrow u_{o}$ in $L^{2}\left(\Omega, \mathbf{R}^{N}\right)$ as $h \downarrow 0$.

In order to prove a corresponding assertion for the case $p>1$ (as well as in some other proofs of the present paper), we need the following technical lemmas.

Lemma 2.12. Let $p \geq 1, \varepsilon>0$ and $v_{o} \in L^{2}\left(\Omega, \mathbf{R}^{N}\right)$. Then, for the regularization $v_{o, \varepsilon}:=v_{o} * \phi_{\varepsilon}$, where $v_{o}$ is extended to $\mathbf{R}^{n}$ by zero and $\phi_{\varepsilon}$ denotes the standard mollifier in $\mathbf{R}^{n}$ we have that

$$
\int_{\Omega}\left|D v_{o, \varepsilon}\right|^{p} \mathrm{~d} x \leq c(n, p,|\Omega|) \varepsilon^{-(n+1) p}\left\|v_{o}\right\|_{L^{2}\left(\Omega, \mathbf{R}^{N}\right)}^{p} .
$$

Proof. By means of Young's inequality for convolutions and Hölder's inequality we estimate

$$
\begin{aligned}
\int_{\Omega}\left|D v_{o, \varepsilon}\right|^{p} \mathrm{~d} x & =\int_{\Omega}\left|v_{o} * D \phi_{\varepsilon}\right|^{p} \mathrm{~d} x \leq\left\|v_{o}\right\|_{L^{1}\left(\Omega, \mathbf{R}^{N}\right)}^{p}\left\|D \phi_{\varepsilon}\right\|_{L^{p}\left(\mathbf{R}^{n}\right)}^{p} \\
& \leq|\Omega|^{\frac{p}{2}}\left\|v_{o}\right\|_{L^{2}\left(\Omega, \mathbf{R}^{N}\right)}^{p} \varepsilon^{-(n+1) p}\|D \phi\|_{L^{p}\left(\mathbf{R}^{n}\right)}^{p} \\
& =c(n, p,|\Omega|) \varepsilon^{-(n+1) p}\left\|v_{o}\right\|_{L^{2}\left(\Omega, \mathbf{R}^{N}\right)}^{p},
\end{aligned}
$$

which yields the claim.

Lemma 2.13. Let $h>0$ and $\varepsilon(h)=h^{\frac{1}{2(n+1) p}}$. Assume that $v_{o} \in L^{2}\left(\Omega, \mathbf{R}^{N}\right)$ and consider the regularization $v_{o, h}:=v_{o} * \phi_{\varepsilon(h)}$, where $v_{o}$ is extended to $\mathbf{R}^{n}$ by zero and $\phi_{\varepsilon(h)}$ denotes the standard mollifier in $\mathbf{R}^{n}$. Suppose that $v \in L^{p}\left(0, T ; W^{1, p}\left(\Omega, \mathbf{R}^{N}\right)\right)$ and let $\llbracket v \rrbracket_{h}$ denote the mollification according to (2.2) with initial datum $v_{o, h}$. Then we have that $D \llbracket v \rrbracket_{h} \rightarrow D v$ in $L^{p}\left(\Omega_{T}, \mathbf{R}^{N \times n}\right)$ in the limit $h \downarrow 0$.

Proof. Consider $\llbracket v \rrbracket_{h}^{o}=\llbracket v \rrbracket_{h}-\mathrm{e}^{-\frac{t}{h}} v_{o, h}$, i.e. the time regularization according to (2.2) with zero initial values. By Lemma 2.7 we know that

$$
D \llbracket v \rrbracket_{h}^{o} \rightarrow D v \quad \text { in } L^{p}\left(\Omega_{T}, \mathbf{R}^{N \times n}\right) \text { as } h \downarrow 0 .
$$

Furthermore, we have that

$$
\iint_{\Omega_{T}}\left|D \mathrm{e}^{-\frac{t}{h}} v_{o, h}\right|^{p} \mathrm{~d} x \mathrm{~d} t=\int_{0}^{T} \mathrm{e}^{-\frac{p t}{h}} \mathrm{~d} t \int_{\Omega}\left|D v_{o, h}\right|^{p} \mathrm{~d} x \leq \frac{h}{p} \int_{\Omega}\left|D v_{o, h}\right|^{p} \mathrm{~d} x .
$$

Using (2.5), we find that

$$
\int_{\Omega}\left|D v_{o, h}\right|^{p} \mathrm{~d} x \leq c(n, p,|\Omega|) \varepsilon(h)^{-(n+1) p}\left\|v_{o}\right\|_{L^{2}\left(\Omega, \mathbf{R}^{N}\right)}^{p} .
$$

Joining the two preceding estimates and recalling the definition of $\varepsilon(h)$, we obtain that

$$
\iint_{\Omega_{T}}\left|D \mathrm{e}^{-\frac{t}{h}} v_{o, h}\right|^{p} \mathrm{~d} x \mathrm{~d} t \leq c(n, p,|\Omega|) \sqrt{h}\left\|v_{o}\right\|_{L^{2}\left(\Omega, \mathbf{R}^{N}\right)}^{p} \rightarrow 0
$$

as $h \downarrow 0$. Together with (2.6) this yields the claim of the lemma. 
Lemma 2.14. Assume that $u_{p, o}$ satisfies (1.16), $f^{p}$ fulfills (1.14) and (1.15) and $g$ fulfills (1.10) and (1.11). Then any variational solution $u$ in the sense of Definition 1.3 attains the initial datum $u_{p, o}$ in the $C^{0}-L^{2}$-sense, i.e.

$$
\lim _{t \downarrow 0}\left\|u(t)-u_{p, o}\right\|_{L^{2}\left(\Omega, \mathbf{R}^{N}\right)}=0 .
$$

Proof. Let $h>0$ and $\varepsilon=\varepsilon(h):=h^{\frac{1}{2(n+1) p}}$. We extend $u_{p, o}$ to $\mathbf{R}^{n}$ by zero and consider the mollification $u_{p, o, h}:=u_{p, o} * \phi_{\varepsilon(h)}$, where $\phi_{\varepsilon(h)}$ denotes the standard mollifier in $\mathbf{R}^{n}$. Since $v \equiv u_{p, o, h}$ is an admissible comparison map in (1.17), we deduce that

$$
\begin{aligned}
& \frac{1}{4}\left\|u(\tau)-u_{p, o}\right\|_{L^{2}\left(\Omega, \mathbf{R}^{N}\right)}^{2} \leq \frac{1}{2}\left\|u(\tau)-u_{p, o, h}\right\|_{L^{2}\left(\Omega, \mathbf{R}^{N}\right)}^{2}+\frac{1}{2}\left\|u_{p, o}-u_{p, o, h}\right\|_{L^{2}\left(\Omega, \mathbf{R}^{N}\right)}^{2} \\
& \leq \tau L \int_{\Omega}\left(1+\left|D u_{p, o, h}\right|^{p}\right) \mathrm{d} x+\tau M \int_{\Omega}\left(1+\left|u_{p, o, h}\right|^{2}\right) \mathrm{d} x+\left\|u_{p, o}-u_{p, o, h}\right\|_{L^{2}\left(\Omega, \mathbf{R}^{N}\right)}^{2}
\end{aligned}
$$

for any $\tau \in[0, T]$. By Lemma 2.12, we obtain for the first term on the right-hand side of the preceding inequality that

$$
\begin{aligned}
\int_{\Omega}\left|D u_{p, o, h}\right|^{p} \mathrm{~d} x & \leq c(n, p,|\Omega|) \varepsilon^{-(n+1) p}\left\|u_{p, o}\right\|_{L^{2}\left(\Omega, \mathbf{R}^{N}\right)} \\
& =c(n, p,|\Omega|) \frac{1}{\sqrt{h}}\left\|u_{p, o}\right\|_{L^{2}\left(\Omega, \mathbf{R}^{N}\right)} .
\end{aligned}
$$

Inserting this in the second last inequality, integrating over $\tau \in(0, h)$ and dividing by $h$ yields

$$
\begin{aligned}
& \frac{1}{4 h} \int_{0}^{h}\left\|u(\tau)-u_{p, o}\right\|_{L^{2}\left(\Omega, \mathbf{R}^{N}\right)}^{2} \mathrm{~d} \tau \\
& \leq \frac{h}{2}(L+M)|\Omega|+c(n, p,|\Omega|, L) \sqrt{h}\left\|u_{p, o}\right\|_{L^{2}\left(\Omega, \mathbf{R}^{N}\right)}+\frac{h}{2} M\left\|u_{p, o, h}\right\|_{L^{2}\left(\Omega, \mathbf{R}^{N}\right)}^{2} \\
& \quad+\left\|u_{p, o}-u_{p, o, h}\right\|_{L^{2}\left(\Omega, \mathbf{R}^{N}\right)}^{2} .
\end{aligned}
$$

The right-hand side of the preceding inequality vanishes as $h \downarrow 0$, since $u_{p, o, h} \rightarrow u_{p, o}$ in $L^{2}\left(\Omega, \mathbf{R}^{N}\right)$ as $h \downarrow 0$. Since $u \in C^{0}\left([0, T] ; L^{2}\left(\Omega, \mathbf{R}^{N}\right)\right)$, this implies the claim.

2.7. Energy bounds. Next, we show certain energy bounds for variational solutions associated with the approximations $f^{p}$ with $p>1$. Using $v \equiv 0$ as comparison map in (1.17) and the growth conditions (1.14) and (1.10), we deduce the following lemma.

Lemma 2.15. Assume that $u_{p, o}$ fulfills (1.16) and that $f^{p}$ satisfies the hypotheses (1.14) and (1.15). Then any variational solution in the sense of Definition 1.3 satisfies the energy bound

$$
\begin{aligned}
& \frac{1}{2} \sup _{t \in[0, T]}\|u(t)\|_{L^{2}\left(\Omega, \mathbf{R}^{N}\right)}^{2}+\iint_{\Omega_{T}}\left[f^{p}(x, D u)+g(x, u)\right] \mathrm{d} x \mathrm{~d} t \\
& \leq 2\left[L_{p}+M\right]\left|\Omega_{T}\right|+\left\|u_{p, o}\right\|_{L^{2}\left(\Omega, \mathbf{R}^{N}\right)}^{2} .
\end{aligned}
$$

The next lemma provides a continuity property that will be crucial for the application of the compactness result Lemma 2.1.

Lemma 2.16. For an exponent $p \in(1,2]$ we consider a Cauchy datum $u_{p, o}$ as in (1.16). Assume that

$$
u \in C^{0}\left([0, T] ; L^{2}\left(\Omega, \mathbf{R}^{N}\right)\right) \cap L^{p}\left(0, T ; W^{1, p}\left(\Omega, \mathbf{R}^{N}\right)\right)
$$


is a variational solution to (1.4) in the sense of Definition 1.3. Then, for any $h \in(0, T)$ and any $\ell \geq 1$ there holds

$$
\int_{0}^{T-h}\|u(t+h)-u(t)\|_{W^{-\ell, 2}\left(\Omega, \mathbf{R}^{N}\right)} \mathrm{d} t \leq c\left[1+\|D u\|_{L^{p}\left(\Omega_{T}, \mathbf{R}^{N}\right)}^{p-1}+\|u\|_{L^{2}\left(\Omega_{T}, \mathbf{R}^{N}\right)}\right] \sqrt{h}
$$

with a constant $c=c(n, N, L, M, \ell,|\Omega|, T)$.

Proof. Let $h \in(0, T)$ and set $\varepsilon(h):=h^{\frac{1}{2(n+1) p}}$. First, we extend $u_{p, o}$ by zero to a function on $\mathbf{R}^{n}$ and define mollifications $u_{p, o, h}:=u_{p, o} * \phi_{\varepsilon(h)}$, where $\phi_{\varepsilon(h)}$ denotes the standard mollifier on $\mathbf{R}^{n}$. We define regularizations $\llbracket u \rrbracket_{h}$ according to (2.2) with initial values $u_{p, o, h}$. Observe that $\llbracket u \rrbracket_{h} \in L^{p}\left(0, T ; W^{1, p}\left(\Omega, \mathbf{R}^{N}\right)\right)$ by Lemma 2.7 , $\partial_{t} \llbracket u \rrbracket_{h} \in L^{1}\left(0, T ; L^{2}\left(\Omega, \mathbf{R}^{N}\right)\right)$ by $(2.3)$ and $\llbracket u \rrbracket_{h}(0)=u_{p, o, h} \in L^{2}\left(\Omega, \mathbf{R}^{N}\right)$. Therefore $v=\llbracket u \rrbracket_{h}+\varphi$, where $\varphi \in C_{0}^{\infty}\left(\Omega_{T}, \mathbf{R}^{N}\right)$, is an admissible comparison map in the variational inequality (1.17). Thus, we have that

$$
\begin{aligned}
\mathrm{I}_{h}:= & -\iint_{\Omega_{T}} \partial_{t}\left(\llbracket u \rrbracket_{h}+\varphi\right) \cdot\left(\llbracket u \rrbracket_{h}-u+\varphi\right) \mathrm{d} x \mathrm{~d} t \\
\leq & \iint_{\Omega_{T}}\left[f^{p}\left(x, D \llbracket u \rrbracket_{h}+D \varphi\right)-f^{p}(x, D u)\right] \mathrm{d} x \mathrm{~d} t \\
& +\iint_{\Omega_{T}}\left[g\left(x, \llbracket u \rrbracket_{h}+\varphi\right)-g(x, u)\right] \mathrm{d} x \mathrm{~d} t+\frac{1}{2}\left\|u_{p, o, h}-u_{p, o}\right\|_{L^{2}\left(\Omega, \mathbf{R}^{N}\right)}^{2} \\
= & : \mathrm{II}_{h}+\mathrm{III}_{h}+\frac{1}{2}\left\|u_{p, o, h}-u_{p, o}\right\|_{L^{2}\left(\Omega, \mathbf{R}^{N}\right)}^{2} .
\end{aligned}
$$

Using the fact that $\varphi(0)=0=\varphi(T)$ we re-write $\mathrm{I}_{h}$ with an integration by parts as follows

$$
\mathrm{I}_{h}=-\iint_{\Omega_{T}} \partial_{t} \llbracket u \rrbracket_{h} \cdot\left(\llbracket u \rrbracket_{h}-u\right) \mathrm{d} x \mathrm{~d} t+\iint_{\Omega_{T}} \partial_{t} \varphi \cdot u \mathrm{~d} x \mathrm{~d} t \geq \iint_{\Omega_{T}} \partial_{t} \varphi \cdot u \mathrm{~d} x \mathrm{~d} t .
$$

In the last inequality we have employed the identity (2.3). Furthermore, we estimate $\mathrm{II}_{h}$ using Lemma 2.10 with the choices $m=N n$ and $p_{o}=2$. This yields

$$
\begin{aligned}
\mathrm{II}_{h}= & \iint_{\Omega_{T}}\left[f^{p}\left(x, D \llbracket u \rrbracket_{h}+D \varphi\right)-f^{p}\left(x, D \llbracket u \rrbracket_{h}\right)+f^{p}\left(x, D \llbracket u \rrbracket_{h}\right)-f^{p}(x, D u)\right] \mathrm{d} x \mathrm{~d} t \\
\leq & c \iint_{\Omega_{T}}\left(1+\left|D \llbracket u \rrbracket_{h}+D \varphi\right|^{p-1}+\left|D \llbracket u \rrbracket_{h}\right|^{p-1}\right)|D \varphi| \mathrm{d} x \mathrm{~d} t \\
& +c \iint_{\Omega_{T}}\left(1+\left|D \llbracket u \rrbracket_{h}\right|^{p-1}+|D u|^{p-1}\right)\left|D \llbracket u \rrbracket_{h}-D u\right| \mathrm{d} x \mathrm{~d} t
\end{aligned}
$$

where $c=c(N n) L_{p}$. By Lemma 2.13 we have that $D \llbracket u \rrbracket_{h} \rightarrow D u$ in $L^{p}\left(\Omega_{T}, \mathbf{R}^{N \times n}\right)$ as $h \downarrow 0$. In conclusion, we infer that

$$
\limsup _{h \downarrow 0} \mathrm{II}_{h} \leq c(N n) L_{p} \iint_{\Omega_{T}}\left(1+|D u|^{p-1}+|D \varphi|^{p-1}\right)|D \varphi| \mathrm{d} x \mathrm{~d} t .
$$

Similarly, applying Lemma 2.10 with $m=N$ and $p_{o}=p=2$, we deduce that

$$
\begin{aligned}
\mathrm{III}_{h}= & \iint_{\Omega_{T}}\left[g\left(x, \llbracket u \rrbracket_{h}+\varphi\right)-g\left(x, \llbracket u \rrbracket_{h}\right)+g\left(x, \llbracket u \rrbracket_{h}\right)-g(x, u)\right] \mathrm{d} x \mathrm{~d} t \\
\leq & c \iint_{\Omega_{T}}\left(1+\left|\llbracket u \rrbracket_{h}+\varphi\right|+\left|\llbracket u \rrbracket_{h}\right|\right)|\varphi| \mathrm{d} x \mathrm{~d} t \\
& +c \iint_{\Omega_{T}}\left(1+\left|\llbracket u \rrbracket_{h}\right|+|u|\right)\left|\llbracket u \rrbracket_{h}-u\right| \mathrm{d} x \mathrm{~d} t
\end{aligned}
$$


where $c=c(N) M$. Since $\llbracket u \rrbracket_{h} \rightarrow u$ in $L^{2}\left(\Omega_{T}, \mathbf{R}^{N}\right)$ as $h \downarrow 0$ by Lemma 2.7 and the fact that $u_{p, o, h} \rightarrow u_{p, o}$ in $L^{2}\left(\Omega, \mathbf{R}^{N}\right)$ as $h \downarrow 0$, we conclude that

$$
\limsup _{h \downarrow 0} \operatorname{III}_{h} \leq c(N) M \iint_{\Omega_{T}}(1+|u|+|\varphi|)|\varphi| \mathrm{d} x \mathrm{~d} t .
$$

Furthermore we have that

$$
\lim _{h \downarrow 0} \frac{1}{2}\left\|u_{p, o, h}-u_{p, o}\right\|_{L^{2}\left(\Omega, \mathbf{R}^{N}\right)}^{2}=0 .
$$

Inserting the preceding computations into (2.8), we have that

$$
\begin{aligned}
\iint_{\Omega_{T}} \partial_{t} \varphi \cdot u \mathrm{~d} x \mathrm{~d} t \leq & c\left(N n, p_{o}\right) L \iint_{\Omega_{T}}\left(1+|D u|^{p-1}+|D \varphi|^{p-1}\right)|D \varphi| \mathrm{d} x \mathrm{~d} t \\
& +c(N) M \iint_{\Omega_{T}}(1+|u|+|\varphi|)|\varphi| \mathrm{d} x \mathrm{~d} t .
\end{aligned}
$$

Repeating the same computation for $-\varphi$ instead of $\varphi$, we obtain that

$$
\begin{aligned}
& \left|\iint_{\Omega_{T}} \partial_{t} \varphi \cdot u \mathrm{~d} x \mathrm{~d} t\right| \\
& \leq c_{1} \iint_{\Omega_{T}}\left(1+|D u|^{p-1}+|D \varphi|^{p-1}\right)|D \varphi| \mathrm{d} x \mathrm{~d} t \\
& \quad+c_{2} \iint_{\Omega_{T}}(1+|u|+|\varphi|)|\varphi| \mathrm{d} x \mathrm{~d} t \\
& \leq c_{1}\left[\left|\Omega_{T}\right|^{1-\frac{1}{p}}+\|D u\|_{L^{p}\left(\Omega_{T}, \mathbf{R}^{N n}\right)}^{p-1}+\|D \varphi\|_{L^{p}\left(\Omega_{T}, \mathbf{R}^{N n}\right)}^{p-1}\right]\|D \varphi\|_{L^{p}\left(\Omega_{T}, \mathbf{R}^{N n}\right)} \\
& \quad+c_{2}\left[\left|\Omega_{T}\right|^{\frac{1}{2}}+\|u\|_{L^{2}\left(\Omega_{T}, \mathbf{R}^{N}\right)}+\|\varphi\|_{L^{2}\left(\Omega_{T}, \mathbf{R}^{N}\right)}\right]\|\varphi\|_{L^{2}\left(\Omega_{T}, \mathbf{R}^{N}\right)} \\
& \leq c_{1}\left|\Omega_{T}\right|^{\frac{1}{p}-\frac{1}{2}}\left[\left|\Omega_{T}\right|^{1-\frac{1}{p}}+\|D u\|_{L^{p}\left(\Omega_{T}, \mathbf{R}^{N n}\right)}^{p-1}+\|D \varphi\|_{L^{p}\left(\Omega_{T}, \mathbf{R}^{N n}\right)}^{p-1}\right]\|D \varphi\|_{L^{2}\left(\Omega_{T}, \mathbf{R}^{N n}\right)} \\
& \quad+c_{2}\left[\left|\Omega_{T}\right|^{\frac{1}{2}}+\|u\|_{L^{2}\left(\Omega_{T}, \mathbf{R}^{N}\right)}+\|\varphi\|_{L^{2}\left(\Omega_{T}, \mathbf{R}^{N}\right)}\right]\|\varphi\|_{L^{2}\left(\Omega_{T}, \mathbf{R}^{N}\right)}
\end{aligned}
$$

holds true for any $\varphi \in C_{0}^{\infty}\left(\Omega_{T}, \mathbf{R}^{N}\right)$ with $c_{1}=c(N n) L_{p}$ and $c_{2}=c(N) M$. An approximation argument yields the same estimate for any function $\varphi \in C_{0}^{0,1}\left(\Omega_{T}, \mathbf{R}^{N}\right)$. Now, for $0<s_{1}<s_{2}<T$ and $0<\sigma<\min \left\{s_{1}, T-s_{2}\right\}$ we define

$$
\zeta_{\sigma}(t):= \begin{cases}0, & \text { for } t \in\left[0, s_{1}-\sigma\right], \\ \frac{1}{\sigma}\left(t-s_{1}+\sigma\right) & \text { for } t \in\left(s_{1}-\sigma, s_{1}\right), \\ 1 & \text { for } t \in\left[s_{1}, s_{2}\right] \\ -\frac{1}{\sigma}\left(t-s_{2}-\sigma\right) & \text { for } t \in\left(s_{2}, s_{2}+\sigma\right), \\ 0, & \text { for } t \in\left[s_{2}+\sigma, T\right],\end{cases}
$$

and choose $\varphi(x, t)=\psi(x) \zeta_{\sigma}(t)$ with $\psi \in C_{0}^{\infty}\left(\Omega, \mathbf{R}^{N}\right)$ in $(2.9)$ with the result that

$$
\left|\int_{\Omega}\left(\frac{1}{\sigma} \int_{s_{1}-\sigma}^{s_{1}} u(x, t) \mathrm{d} t-\frac{1}{\sigma} \int_{s_{2}}^{s_{2}+\sigma} u(x, t) \mathrm{d} t\right) \cdot \psi(x) \mathrm{d} x\right|
$$




$$
\begin{aligned}
\leq & c_{1}\left|\Omega_{T}\right|^{\frac{1}{p}-\frac{1}{2}}\left[\left|\Omega_{T}\right|^{1-\frac{1}{p}}+\|D u\|_{L^{p}\left(\Omega_{T}, \mathbf{R}^{N n}\right)}^{p-1}+T^{1-\frac{1}{p}}\|D \psi\|_{L^{p}\left(\Omega, \mathbf{R}^{N n}\right)}^{p-1}\right] \\
& \cdot\left(s_{2}-s_{1}+2 \sigma\right)^{\frac{1}{2}}\|D \psi\|_{L^{2}\left(\Omega, \mathbf{R}^{N n}\right)} \\
+ & c_{2}\left[\left|\Omega_{T}\right|^{\frac{1}{2}}+\|u\|_{L^{2}\left(\Omega_{T}, \mathbf{R}^{N}\right)}+T^{\frac{1}{2}}\|\psi\|_{L^{2}\left(\Omega, \mathbf{R}^{N}\right)}\right]\left(s_{2}-s_{1}+2 \sigma\right)^{\frac{1}{2}}\|\psi\|_{L^{2}\left(\Omega, \mathbf{R}^{N}\right)}
\end{aligned}
$$

holds true. By Sobolev's embedding we thus obtain for $\ell \geq 1$ that

$$
\begin{aligned}
& \left|\int_{\Omega}\left(\frac{1}{\sigma} \int_{s_{1}-\sigma}^{s_{1}} u(x, t) \mathrm{d} t-\frac{1}{\sigma} \int_{s_{2}}^{s_{2}+\sigma} u(x, t) \mathrm{d} t\right) \cdot \psi(x) \mathrm{d} x\right| \\
& \leq c\left[1+\|D u\|_{L^{p}\left(\Omega_{T}, \mathbf{R}^{N n}\right)}^{p-1}+\|u\|_{L^{2}\left(\Omega_{T}, \mathbf{R}^{N}\right)}+\|\psi\|_{L^{2}\left(\Omega, \mathbf{R}^{N}\right)}+\|D \psi\|_{L^{p}\left(\Omega, \mathbf{R}^{N n}\right)}^{p-1}\right] \\
& \cdot\left(s_{2}-s_{1}+2 \sigma\right)^{\frac{1}{2}}\|\psi\|_{W^{\ell, 2}\left(\Omega, \mathbf{R}^{N}\right)},
\end{aligned}
$$

where $c=c(n, N, L, M, \ell,|\Omega|, T)$. Using this estimate for $\delta \psi$ with $\delta>0$ instead of $\psi$, dividing by $\delta>0$ and letting $\delta \downarrow 0$, the preceding estimate simplifies to

$$
\begin{aligned}
& \left|\int_{\Omega}\left(\frac{1}{\sigma} \int_{s_{1}-\sigma}^{s_{1}} u(x, t) \mathrm{d} t-\frac{1}{\sigma} \int_{s_{2}}^{s_{2}+\sigma} u(x, t) \mathrm{d} t\right) \cdot \psi(x) \mathrm{d} x\right| \\
& \leq c\left[1+\|D u\|_{L^{p}\left(\Omega_{T}, \mathbf{R}^{N n}\right)}^{p-1}+\|u\|_{L^{2}\left(\Omega_{T}, \mathbf{R}^{N}\right)}\right]\left(s_{2}-s_{1}+2 \sigma\right)^{\frac{1}{2}}\|\psi\|_{W^{\ell, 2}\left(\Omega, \mathbf{R}^{N}\right)} .
\end{aligned}
$$

Passing to the limit $\sigma \downarrow 0$ we find that for every $0<s_{1}<s_{2}<T$ there holds

$$
\begin{aligned}
& \left|\int_{\Omega}\left(u\left(x, s_{2}\right)-u\left(x, s_{1}\right)\right) \cdot \psi(x) \mathrm{d} x\right| \\
& \leq c\left[1+\|D u\|_{L^{p}\left(\Omega_{T}, \mathbf{R}^{N n}\right)}^{p-1}+\|u\|_{L^{2}\left(\Omega_{T}, \mathbf{R}^{N}\right)}\right]\left(s_{2}-s_{1}\right)^{\frac{1}{2}}\|\psi\|_{W^{\ell, 2}\left(\Omega, \mathbf{R}^{N}\right)}
\end{aligned}
$$

for any $\psi \in C_{0}^{\infty}\left(\Omega, \mathbf{R}^{N}\right)$. By density of $C_{0}^{\infty}\left(\Omega, \mathbf{R}^{N}\right)$ in $W_{0}^{\ell, 2}\left(\Omega, \mathbf{R}^{N}\right)$ the last inequality extends to any $\psi \in W_{0}^{\ell, 2}\left(\Omega, \mathbf{R}^{N}\right)$. This shows that for any $h \in(0, T)$ we have that

$$
\int_{0}^{T-h}\|u(t+h)-u(t)\|_{W^{-\ell, 2}\left(\Omega, \mathbf{R}^{N}\right)} \mathrm{d} t \leq c\left[1+\|D u\|_{L^{p}\left(\Omega_{T}, \mathbf{R}^{N}\right)}^{p-1}+\|u\|_{L^{2}\left(\Omega_{T}, \mathbf{R}^{N}\right)}\right] \sqrt{h}
$$

holds true with a constant $c=c(n, N, L, M, \ell,|\Omega|, T)$.

\section{Existence of variational solutions for problems with superlinear growth: The case of regular initial data}

In this section, we first consider a regular Cauchy datum $u_{o}$. By regular we mean that $u_{o}$ satisfies

$$
u_{o} \in L^{2}\left(\Omega, \mathbf{R}^{N}\right) \cap W^{1, p}\left(\Omega, \mathbf{R}^{N}\right)
$$

and use the method of minimizing movements to show the existence of variational solutions to the gradient flow associated with

$$
\partial_{t} u-\operatorname{div}\left(D_{\xi} f^{p}(x, D u)\right)=-D_{u} g(x, u) \quad \text { in } \Omega_{T}
$$

in the following theorem. Subsequently, we prove an existence result with a general Cauchy datum in $L^{2}\left(\Omega, \mathbf{R}^{N}\right)$, cf. Theorem 4.1 . 
Theorem 3.1. Suppose that $u_{o}$ is as in (3.1), that $f^{p}$ satisfies hypotheses (1.14) and (1.15) and $g$ satisfies hypotheses (1.10) and (1.11). Then there exists a variational solution $u \in C^{0}\left([0, T] ; L^{2}\left(\Omega, \mathbf{R}^{N}\right)\right) \cap L^{p}\left(0, T ; W^{1, p}\left(\Omega, \mathbf{R}^{N}\right)\right)$ to the gradient flow associated with (3.2) in the sense of Definition 1.3. Furthermore, $u$ satisfies the energy bound

$$
\begin{aligned}
& \frac{1}{2} \iint_{\Omega_{T}}\left|\partial_{t} u\right|^{2} \mathrm{~d} x \mathrm{~d} t+\nu^{p} \sup _{t \in[0, T]} \int_{\Omega \times\{t\}}|D u|^{p} \mathrm{~d} x \mathrm{~d} t \\
& \leq 2\left[\left(L_{p}+M\right)|\Omega|+L_{p}\left\|D u_{o}\right\|_{L^{p}\left(\Omega, \mathbf{R}^{N n}\right)}^{p}+M\left\|u_{o}\right\|_{L^{2}\left(\Omega, \mathbf{R}^{N}\right)}^{2}\right] .
\end{aligned}
$$

The proof of Theorem 3.1 is divided into several steps.

3.1. A sequence of minimizers to elliptic variational functionals. First, we fix a step size $h \in(0,1]$ and consider such values $i \in \mathbf{N}_{0}$ that fulfill $i h \leq T$. We inductively construct minimizers $u_{i} \in L^{2}\left(\Omega, \mathbf{R}^{N}\right) \cap W^{1, p}\left(\Omega, \mathbf{R}^{N}\right)$ to certain elliptic variational functionals. More precisely, let $u_{0}=u_{o}$. If $u_{i-1} \in L^{2}\left(\Omega, \mathbf{R}^{N}\right) \cap W^{1, p}\left(\Omega, \mathbf{R}^{N}\right)$ for some $i \in \mathbf{N}$ has already been constructed, define $u_{i}$ as the minimizer of

$$
\mathrm{I}_{i}[v]:=\int_{\Omega}\left[f^{p}(x, D v)+g(x, v)\right] \mathrm{d} x+\frac{1}{2 h} \int_{\Omega}\left|v-u_{i-1}\right|^{2} \mathrm{~d} x
$$

in the class of functions $v \in L^{2}\left(\Omega, \mathbf{R}^{N}\right) \cap W^{1, p}\left(\Omega, \mathbf{R}^{N}\right)$. Obviously, this class is nonempty. We remark that while this is not the point of view in the present paper, each $\mathrm{I}_{i}$ is a Moreau-Yosida approximation of $\mathbf{I}_{p}$. The existence of a minimizer to $\mathrm{I}_{i}$ in $L^{2}\left(\Omega, \mathbf{R}^{N}\right) \cap W^{1, p}\left(\Omega, \mathbf{R}^{N}\right)$ is ensured by the Direct Method of the Calculus of Variations. Indeed, suppose that $\left(u_{k}\right)_{k \in \mathbf{N}}$ is a minimizing sequence, i.e. $\lim _{k \rightarrow \infty} \mathrm{I}_{i}\left[u_{k}\right]=$ $\inf _{v \in L^{2}\left(\Omega, \mathbf{R}^{N}\right) \cap W^{1, p}\left(\Omega, \mathbf{R}^{N}\right)} \mathrm{I}_{i}[v]$. In particular $\left(\mathrm{I}_{i}\left[u_{k}\right]\right)_{k \in \mathbf{N}}$ is bounded, since $\mathrm{I}_{i}$ is nonnegative by (1.14) and (1.10). If $p \leq 2$, we conclude by the coercivity condition $(1.14)_{1}$ and Hölder's inequality that $\left(u_{k}\right)_{k \in \mathbf{N}}$ is bounded in $L^{2}\left(\Omega, \mathbf{R}^{N}\right) \cap W^{1, p}\left(\Omega, \mathbf{R}^{N}\right)$. If $p>2$, we argue by Poincaré's inequality. More precisely, since $\Omega$ is a bounded Lipschitz domain, there exists a constant $c=c(n, N, p, \Omega)$ such that for any $u \in$ $W^{1, p}\left(\Omega, \mathbf{R}^{N}\right)$

$$
\int_{\Omega}\left|u-u_{\Omega}\right|^{p} \mathrm{~d} x \leq c \int_{\Omega}|D u|^{p} \mathrm{~d} x
$$

holds true, where $u_{\Omega}$ denotes the mean value $u_{\Omega}:=f_{\Omega} u \mathrm{~d} x$. Using Poincaré's inequality and Hölder's inequality, we infer that

$$
\left\|u_{k}\right\|_{L^{p}\left(\Omega, \mathbf{R}^{N}\right)} \leq|\Omega|^{\frac{1}{p}-\frac{1}{2}}\left(\left\|u_{k}-u_{i-1}\right\|_{L^{2}\left(\Omega, \mathbf{R}^{N}\right)}+\left\|u_{i-1}\right\|_{L^{2}\left(\Omega, \mathbf{R}^{N}\right)}\right)+c\left\|D u_{k}\right\|_{L^{p}\left(\Omega, \mathbf{R}^{N n}\right)} .
$$

The right-hand side of the preceding inequality is bounded by $(1.14)_{1}$, since $\left(\mathrm{I}_{i}\left[u_{k}\right]\right)_{k \in \mathbf{N}}$ is bounded and since $u_{i-1} \in L^{2}\left(\Omega, \mathbf{R}^{N}\right)$ is fixed. Hence $\left(u_{k}\right)_{k \in \mathbf{N}}$ is bounded in $L^{2}\left(\Omega, \mathbf{R}^{N}\right) \cap W^{1, p}\left(\Omega, \mathbf{R}^{N}\right)$. Since (1.14), (1.15) and (1.10) together yield that $\mathrm{I}_{i}$ is lower semicontinuous with respect to weak convergence in $L^{2}\left(\Omega, \mathbf{R}^{N}\right) \cap W^{1, p}\left(\Omega, \mathbf{R}^{N}\right)$, there exists a minimizer $u$ of $\mathrm{I}_{i}$ in the class $L^{2}\left(\Omega, \mathbf{R}^{N}\right) \cap W^{1, p}\left(\Omega, \mathbf{R}^{N}\right)$.

3.2. Energy estimates. Observe that $u_{i-1}$ is an admissible comparison map for the variational integral $\mathrm{I}_{i}$ for any $i \in \mathbf{N}$. Using the minimality of $u_{i}$ with respect to $\mathrm{I}_{i}$, we obtain that

$$
\begin{aligned}
\mathrm{I}_{i}\left[u_{i}\right] & =\int_{\Omega}\left[f^{p}\left(x, D u_{i}\right)+g\left(x, u_{i}\right)\right] \mathrm{d} x+\frac{1}{2 h} \int_{\Omega}\left|u_{i}-u_{i-1}\right|^{2} \mathrm{~d} x \\
& \leq \int_{\Omega}\left[f^{p}\left(x, D u_{i-1}\right)+g\left(x, u_{i-1}\right)\right] \mathrm{d} x=\mathrm{I}_{i}\left[u_{i-1}\right] .
\end{aligned}
$$


Let $k \in \mathbf{N}$ with $k h \leq T$. Iterating this estimate for $i=1, \ldots, k$ and using the growth conditions (1.14) and (1.10), we conclude that

$$
\begin{aligned}
& \int_{\Omega}\left[f^{p}\left(x, D u_{k}\right)+g\left(x, u_{k}\right)\right] \mathrm{d} x+\frac{1}{2 h} \sum_{i=1}^{k} \int_{\Omega}\left|u_{i}-u_{i-1}\right|^{2} \mathrm{~d} x \\
& \leq \int_{\Omega}\left[f^{p}\left(x, D u_{o}\right)+g\left(x, u_{o}\right)\right] \mathrm{d} x \\
& \leq\left(L_{p}+M\right)|\Omega|+L_{p}\left\|D u_{o}\right\|_{L^{p}\left(\Omega, \mathbf{R}^{N n}\right)}^{p}+M\left\|u_{o}\right\|_{L^{2}\left(\Omega, \mathbf{R}^{N}\right)}^{2}=: \Psi,
\end{aligned}
$$

where the definition of the quantity $\Psi$ is obvious. Discarding the non-negative first term on the left-hand side of (3.4), we compute that

$$
\begin{aligned}
\int_{\Omega}\left|u_{\ell}\right|^{2} \mathrm{~d} x & \leq \int_{\Omega} 2\left[\sum_{i=1}^{\ell}\left|u_{i}-u_{i-1}\right|\right]^{2} \mathrm{~d} x+2 \int_{\Omega}\left|u_{o}\right|^{2} \mathrm{~d} x \\
& \leq 2 \ell \sum_{i=1}^{\ell} \int_{\Omega}\left|u_{i}-u_{i-1}\right|^{2} \mathrm{~d} x+2 \int_{\Omega}\left|u_{o}\right|^{2} \mathrm{~d} x \\
& \leq 4 \ell h \Psi+2\left\|u_{o}\right\|_{L^{2}\left(\Omega, \mathbf{R}^{N}\right)}^{2} \leq 4 T \Psi+2\left\|u_{o}\right\|_{L^{2}\left(\Omega, \mathbf{R}^{N}\right)}^{2} .
\end{aligned}
$$

3.3. The limit map. From now on, we consider only such values $h_{\ell} \in(0,1]$ that are given by $h_{\ell}:=T / \ell$ for some $\ell \in \mathbf{N}$. We assemble the minimizers $u_{i}$ to a function $u^{(\ell)}: \Omega \times\left(-h_{\ell}, T\right] \rightarrow \mathbf{R}^{N}$ that is piecewise constant with respect to time by setting

$$
u^{(\ell)}(\cdot, t):=u_{i} \text { for } t \in\left((i-1) h_{\ell}, i h_{\ell}\right], i \in\left\{0, \ldots, h_{\ell}\right\} .
$$

From $(1.10),(1.14),(3.4)$ and (3.5) we infer the energy estimate

$$
\begin{aligned}
& \sup _{t \in[0, T]}\left\|u^{(\ell)}(t)\right\|_{L^{2}\left(\Omega, \mathbf{R}^{N}\right)}+\nu^{p} \sup _{t \in[0, T]} \int_{\Omega \times\{t\}}\left|D u^{(\ell)}\right|^{p} \mathrm{~d} x \\
& \leq(1+4 T) \Psi+2\left\|u_{o}\right\|_{L^{2}\left(\Omega, \mathbf{R}^{N}\right)}^{2} .
\end{aligned}
$$

We need to conclude that the sequence $\left(u^{(\ell)}\right)_{\ell \in \mathbf{N}}$ is bounded in $L^{\infty}\left(0, T ; L^{2}\left(\Omega, \mathbf{R}^{N}\right)\right) \cap$ $L^{\infty}\left(0, T ; W^{1, p}\left(\Omega, \mathbf{R}^{N}\right)\right)$. If $p \leq 2$, this obviously holds true by (3.7). If $p>2$, we use Poincaré's inequality and Hölder's inequality to infer that

$$
\begin{aligned}
& \sup _{t \in[0, T]}\left\|u^{(\ell)}(t)\right\|_{L^{p}\left(\Omega, \mathbf{R}^{N}\right)} \\
& \leq \sup _{t \in[0, T]}\left\|\left(u^{(\ell)}(t)\right)_{\Omega}\right\|_{L^{p}\left(\Omega, \mathbf{R}^{N}\right)}+\sup _{t \in[0, T]}\left\|u^{(\ell)}(t)-\left(u^{(\ell)}(t)\right)_{\Omega}\right\|_{L^{p}\left(\Omega, \mathbf{R}^{N}\right)} \\
& \leq|\Omega|^{\frac{1}{p}-1} \sup _{t \in[0, T]}\left|\int_{\Omega} u^{(\ell)}(t) \mathrm{d} x\right|+c \sup _{t \in[0, T]}\left\|D u^{(\ell)}(t)\right\|_{L^{p}\left(\Omega, \mathbf{R}^{N n}\right)} \\
& \leq|\Omega|^{\frac{1}{p}-\frac{1}{2}} \sup _{t \in[0, T]}\left\|u^{(\ell)}(t)\right\|_{L^{2}\left(\Omega, \mathbf{R}^{N}\right)}+c \sup _{t \in[0, T]}\left\|D u^{(\ell)}(t)\right\|_{L^{p}\left(\Omega, \mathbf{R}^{N n}\right)},
\end{aligned}
$$

where $c=c(n, N, p, \Omega)$ and $\left(u^{(\ell)}(t)\right)_{\Omega}:=f_{\Omega} u^{(\ell)}(t) \mathrm{d} x$. Together with estimate (3.7) the preceding inequality implies that $\left(u^{(\ell)}\right)_{\ell \in \mathbf{N}}$ is bounded in $L^{\infty}\left(0, T ; L^{2}\left(\Omega, \mathbf{R}^{N}\right)\right) \cap$ $L^{\infty}\left(0, T ; W^{1, p}\left(\Omega, \mathbf{R}^{N}\right)\right)$. Hence, there exists a subsequence $\mathfrak{K} \subset \mathbf{N}$ and a limit map $u \in L^{\infty}\left(0, T ; L^{2}\left(\Omega, \mathbf{R}^{N}\right)\right) \cap L^{p}\left(0, T ; W^{1, p}\left(\Omega, \mathbf{R}^{N}\right)\right)$ such that

$$
\begin{cases}u^{(\ell)} \stackrel{*}{\rightarrow} u & \text { weakly* in } L^{\infty}\left(0, T ; L^{2}\left(\Omega, \mathbf{R}^{N}\right)\right), \\ u^{(\ell)} \stackrel{*}{\rightarrow} u & \text { weakly* in } L^{\infty}\left(0, T ; W^{1, p}\left(\Omega, \mathbf{R}^{N}\right)\right)\end{cases}
$$


in the limit $\mathfrak{K} \ni \ell \rightarrow \infty$. Next, we want to improve the regularity of $u$. To this end, we define a second function $\tilde{u}^{(\ell)}: \Omega \times\left(-h_{\ell}, T\right] \rightarrow \mathbf{R}^{N}$ by linearly interpolating in time the minimizers $u_{i-1}$ and $u_{i}$ on the interval $\left((i-1) h_{\ell}, i h_{\ell}\right]$, i.e. by letting

$$
\tilde{u}^{(\ell)}(\cdot, t):=\left(i-\frac{t}{h_{\ell}}\right) u_{i-1}+\left(1-i+\frac{t}{h_{\ell}}\right) u_{i} \quad \text { for } t \in\left((i-1) h_{\ell}, i h_{\ell}\right], i \in\left\{1, \ldots, h_{\ell}\right\}
$$

and $\tilde{u}^{(\ell)}(\cdot, t):=u_{o}$ for $t \in\left(-h_{\ell}, 0\right]$. Observe that for $t \in\left((i-1) h_{\ell}, i h_{\ell}\right]$ the time derivative of $\tilde{u}^{(\ell)}$ is given by

$$
\partial_{t} \tilde{u}^{(\ell)}(\cdot, t)=\frac{1}{h_{\ell}}\left(u_{i-1}-u_{i}\right) \in L^{2}\left(\Omega, \mathbf{R}^{N}\right)
$$

Together with the coercivity conditions (1.10), (1.14) and the energy estimates (3.4) and (3.5) this implies that

$$
\begin{aligned}
& \frac{1}{2} \iint_{\Omega_{T}}\left|\partial_{t} \tilde{u}^{(\ell)}\right|^{2} \mathrm{~d} x \mathrm{~d} t+\sup _{t \in[0, T]}\left\|\tilde{u}^{(\ell)}(t)\right\|_{L^{2}\left(\Omega, \mathbf{R}^{N}\right)}+\nu^{p} \sup _{t \in[0, T]} \int_{\Omega \times\{t\}}\left|D \tilde{u}^{(\ell)}\right|^{p} \mathrm{~d} x \\
& \leq(2+4 T) \Psi+2\left\|u_{o}\right\|_{L^{2}\left(\Omega, \mathbf{R}^{N}\right)}^{2} .
\end{aligned}
$$

Hence, using Poincaré's inequality in the same way as in the case of $u^{(\ell)}$, by (3.9) also the sequence $\left(\tilde{u}^{(\ell)}\right)_{\ell \in \mathbf{N}}$ is bounded in $L^{\infty}\left(0, T ; W^{1, p}\left(\Omega, \mathbf{R}^{N}\right)\right)$. Furthermore, by (3.9) the sequence of time derivatives $\left(\partial_{t} \tilde{u}^{(\ell)}\right)_{\ell \in \mathbf{N}}$ is bounded in $L^{2}\left(\Omega_{T}, \mathbf{R}^{N}\right)$ and therefore $\left(\tilde{u}^{(\ell)}\right)_{\ell \in \mathbf{N}}$ is bounded in $W^{1, \min \{2, p\}}\left(\Omega_{T}, \mathbf{R}^{N}\right)$. Note that we may apply Rellich's theorem, since $\Omega_{T}$ is a bounded Lipschitz domain. In conclusion, we obtain that there exists a subsequence $\mathfrak{K} \subset \mathbf{N}$ and a limit map $\tilde{u} \in L^{\infty}\left(0, T ; W^{1, p}\left(\Omega, \mathbf{R}^{N}\right)\right)$ with $\partial_{t} \tilde{u} \in L^{2}\left(\Omega_{T}, \mathbf{R}^{N}\right)$ such that

$$
\begin{cases}\tilde{u}^{(\ell)} \rightarrow \tilde{u} & \text { strongly in } L^{\min \{2, p\}}\left(\Omega_{T}, \mathbf{R}^{N}\right), \\ \tilde{u}^{(\ell)} * \tilde{u} & \text { weakly* in } L^{\infty}\left(0, T ; W^{1, p}\left(\Omega, \mathbf{R}^{N}\right)\right), \\ \partial_{t} \tilde{u}^{(\ell)} \rightarrow \partial_{t} \tilde{u} & \text { weakly in } L^{2}\left(\Omega_{T}, \mathbf{R}^{N}\right)\end{cases}
$$

in the limit $\mathfrak{K} \ni \ell \rightarrow \infty$. Since

$$
\left|\left(\tilde{u}^{(\ell)}-u^{(\ell)}\right)(t)\right| \leq\left|u_{i-1}-u_{i}\right| \text { for } t \in\left((i-1) h_{\ell}, i h_{\ell}\right]
$$

we conclude from (3.4) that

$$
\iint_{\Omega_{T}}\left|\left(\tilde{u}^{(\ell)}-u^{(\ell)}\right)(t)\right|^{2} \mathrm{~d} x \mathrm{~d} t \leq h_{\ell} \sum_{i=1}^{\ell} \int_{\Omega}\left|u_{i-1}-u_{i}\right|^{2} \mathrm{~d} x \leq 2 h_{\ell}^{2} \Psi
$$

and further by means of Hölder's inequality that

$$
\iint_{\Omega_{T}}\left|\left(\tilde{u}^{(\ell)}-u^{(\ell)}\right)(t)\right|^{\min \{2, p\}} \mathrm{d} x \mathrm{~d} t \leq\left|\Omega_{T}\right|^{1-\frac{\min \{2, p\}}{2}}\left(2 h_{\ell}^{2} \Psi\right)^{\frac{\min \{2, p\}}{2}} .
$$

Joining this with $(3.10)_{1}$ implies that also $u^{(\ell)} \rightarrow \tilde{u}$ strongly in $L^{\min \{2, p\}}\left(\Omega_{T}, \mathbf{R}^{N}\right)$ as $\mathfrak{K} \ni \ell \rightarrow \infty$ and hence $u=\tilde{u}$. At this stage, we are able to deduce the energy bound (3.3). To this end note that (3.4) leads to

$$
\begin{aligned}
& \frac{1}{2} \iint_{\Omega_{T}}\left|\partial_{t} \tilde{u}^{(\ell)}\right|^{2} \mathrm{~d} x \mathrm{~d} t+\nu^{p} \sup _{t \in[0, T]} \int_{\Omega \times\{t\}}\left|D \tilde{u}^{(\ell)}\right|^{p} \mathrm{~d} x \\
& \leq 2\left[\left(L_{p}+M\right)|\Omega|+L_{p}\left\|D u_{o}\right\|_{L^{p}\left(\Omega, \mathbf{R}^{N n}\right)}^{p}+M\left\|u_{o}\right\|_{L^{2}\left(\Omega, \mathbf{R}^{N}\right)}^{2}\right] .
\end{aligned}
$$

By weak lower semicontinuity, (3.3) follows from taking the limit $\mathfrak{K} \ni \ell \rightarrow \infty$ in the preceding inequality. 
3.4. Minimizing property of the approximations. First, observe that $u^{(\ell)}$ is a minimizer of the variational functional

$$
\mathbf{I}^{(\ell)}[v]:=\iint_{\Omega_{T}}\left[f^{p}(x, D v)+g(x, v)+\frac{1}{2 h_{\ell}}\left|v-u^{(\ell)}\left(t-h_{\ell}\right)\right|\right] \mathrm{d} x \mathrm{~d} t
$$

in the class of functions $v \in L^{2}\left(\Omega_{T}, \mathbf{R}^{N}\right) \cap L^{p}\left(0, T ; W^{1, p}\left(\Omega, \mathbf{R}^{N}\right)\right)$. Indeed, using the definitions of $\mathbf{I}^{(\ell)}$ and $u^{(\ell)}$ and the minimality of $u_{i}$ with respect to $\mathrm{I}_{i}$, we compute that

$$
\begin{aligned}
\mathbf{I}^{(\ell)}\left[u^{(\ell)}\right] & =\sum_{i=1}^{\ell} \int_{(i-1) h_{\ell}}^{h_{\ell}} \int_{\Omega}\left[f^{p}\left(x, D u_{i}\right)+g\left(x, u_{i}\right)+\frac{1}{2 h_{\ell}}\left|u_{i}-u_{i-1}\right|^{2}\right] \mathrm{d} x \mathrm{~d} t \\
& =\sum_{i=1}^{\ell} \int_{(i-1) h_{\ell}}^{h_{\ell}} \mathrm{I}_{i}\left[u_{i}\right] \mathrm{d} t \leq \sum_{i=1}^{\ell} \int_{(i-1) h_{\ell}}^{h_{\ell}} \mathrm{I}_{i}[v(t)] \mathrm{d} t \\
& =\sum_{i=1}^{\ell} \int_{(i-1) h_{\ell}}^{h_{\ell}} \int_{\Omega}\left[f^{p}(x, D v)+g(x, v)+\frac{1}{2 h_{\ell}}\left|v-u_{i-1}\right|^{2}\right] \mathrm{d} x \mathrm{~d} t=\mathbf{I}^{(\ell)}[v]
\end{aligned}
$$

holds true for any map $v \in L^{2}\left(\Omega_{T}, \mathbf{R}^{N}\right) \cap L^{p}\left(0, T ; W^{1, p}\left(\Omega, \mathbf{R}^{N}\right)\right)$. We re-write the preceding inequality as

$$
\begin{aligned}
& \iint_{\Omega_{T}}\left[f^{p}\left(x, D u^{(\ell)}\right)+g\left(x, u^{(\ell)}\right)\right] \mathrm{d} x \mathrm{~d} t \\
& \leq \iint_{\Omega_{T}}\left[f^{p}(x, D v)+g(x, v)\right] \mathrm{d} x \mathrm{~d} t \\
& \quad+\frac{1}{2 h_{\ell}} \iint_{\Omega_{T}}\left[\left|v-u^{(\ell)}\left(t-h_{\ell}\right)\right|^{2}-\left|u^{(\ell)}-u^{(\ell)}\left(t-h_{\ell}\right)\right|^{2}\right] \mathrm{d} x \mathrm{~d} t \\
& =\iint_{\Omega_{T}}\left[f^{p}(x, D v)+g(x, v)\right] \mathrm{d} x \mathrm{~d} t \\
& \quad+\frac{1}{h_{\ell}} \iint_{\Omega_{T}}\left[\frac{1}{2}\left|v-u^{(\ell)}\right|^{2}+\left(v-u^{(\ell)}\right) \cdot\left(u^{(\ell)}-u^{(\ell)}\left(t-h_{\ell}\right)\right)\right] \mathrm{d} x \mathrm{~d} t .
\end{aligned}
$$

Next, using the (admissible) convex combination $w^{(\ell)}:=u^{(\ell)}+s\left(v-u^{(\ell)}\right)$ with $s \in$ $(0,1)$ as comparison map in the preceding inequality, we deduce by the convexity assumptions (1.11) and (1.15) that

$$
\begin{aligned}
& \iint_{\Omega_{T}}\left[f^{p}\left(x, D u^{(\ell)}\right)+g\left(x, u^{(\ell)}\right)\right] \mathrm{d} x \mathrm{~d} t \\
& \leq \iint_{\Omega_{T}}\left[f^{p}\left(x, D u^{(\ell)}+s\left(D v-D u^{(\ell)}\right)\right)+g\left(x, u^{(\ell)}+s\left(v-u^{(\ell)}\right)\right)\right] \mathrm{d} x \mathrm{~d} t \\
& \quad+\frac{1}{h_{\ell}} \iint_{\Omega_{T}}\left[\frac{s^{2}}{2}\left|v-u^{(\ell)}\right|^{2}+s\left(v-u^{(\ell)}\right) \cdot\left(u^{(\ell)}-u^{(\ell)}\left(t-h_{\ell}\right)\right)\right] \mathrm{d} x \mathrm{~d} t \\
& \leq \iint_{\Omega_{T}}\left[(1-s)\left[f^{p}\left(x, D u^{(\ell)}\right)+g\left(x, u^{(\ell)}\right)\right]+s\left[f^{p}(x, D v)+g(x, v)\right]\right] \mathrm{d} x \mathrm{~d} t \\
& \quad+\frac{1}{h_{\ell}} \iint_{\Omega_{T}}\left[\frac{s^{2}}{2}\left|v-u^{(\ell)}\right|^{2}+s\left(v-u^{(\ell)}\right) \cdot\left(u^{(\ell)}-u^{(\ell)}\left(t-h_{\ell}\right)\right)\right] \mathrm{d} x \mathrm{~d} t .
\end{aligned}
$$


Reabsorbing the first term on the right-hand side of the preceding inequality into the left-hand side and dividing the result by $s>0$, we infer that

$$
\begin{aligned}
& \iint_{\Omega_{T}}\left[f^{p}\left(x, D u^{(\ell)}\right)+g\left(x, u^{(\ell)}\right)\right] \mathrm{d} x \mathrm{~d} t \leq \iint_{\Omega_{T}}\left[f^{p}(x, D v)+g(x, v)\right] \mathrm{d} x \mathrm{~d} t \\
& \quad+\frac{1}{h_{\ell}} \iint_{\Omega_{T}}\left[\frac{s}{2}\left|v-u^{(\ell)}\right|^{2}+\left(v-u^{(\ell)}\right) \cdot\left(u^{(\ell)}-u^{(\ell)}\left(t-h_{\ell}\right)\right)\right] \mathrm{d} x \mathrm{~d} t .
\end{aligned}
$$

We extend $v$ to negative times $t<0$ by $v(t)=v(0) \in L^{2}\left(\Omega, \mathbf{R}^{N}\right)$. Passing to the limit $s \downarrow 0$ in the preceding inequality, we find that the variational inequality

$$
\begin{aligned}
& \iint_{\Omega_{T}}\left[f^{p}\left(x, D u^{(\ell)}\right)+g\left(x, u^{(\ell)}\right)\right] \mathrm{d} x \mathrm{~d} t \\
& \leq \iint_{\Omega_{T}}\left[f^{p}(x, D v)+g(x, v)\right] \mathrm{d} x \mathrm{~d} t \\
&\left.+\frac{1}{h_{\ell}} \iint_{\Omega_{T}}\left(v-u^{(\ell)}\right) \cdot\left(u^{(\ell)}-u^{(\ell)}\left(t-h_{\ell}\right)\right)\right] \mathrm{d} x \mathrm{~d} t \\
&= \iint_{\Omega_{T}}\left[f^{p}(x, D v)+g(x, v)\right] \mathrm{d} x \mathrm{~d} t+\frac{1}{h_{\ell}} \iint_{\Omega_{T}}\left(v-u^{(\ell)}\right) \cdot\left(v-v\left(t-h_{\ell}\right)\right) \mathrm{d} x \mathrm{~d} t \\
&+\frac{1}{2 h_{\ell}} \iint_{\Omega_{T}}\left|v-u^{(\ell)}\right|^{2}\left(t-h_{\ell}\right)-\left|v-u^{(\ell)}\right|^{2} \mathrm{~d} x \mathrm{~d} t \\
&-\frac{1}{2 h_{\ell}} \iint_{\Omega_{T}}\left|v-v\left(t-h_{\ell}\right)-u^{(\ell)}+u^{(\ell)}\left(t-h_{\ell}\right)\right|^{2} \mathrm{~d} x \mathrm{~d} t \\
& \leq \iint_{\Omega_{T}}\left[f^{p}(x, D v)+g(x, v)\right] \mathrm{d} x \mathrm{~d} t+\frac{1}{h_{\ell}} \iint_{\Omega_{T}}\left(v-u^{(\ell)}\right) \cdot\left(v-v\left(t-h_{\ell}\right)\right) \mathrm{d} x \mathrm{~d} t \\
&-\frac{1}{2 h_{\ell}} \iint_{\Omega \times\left[T-h_{\ell}, T\right]}\left|v-u^{(\ell)}\right|^{2} \mathrm{~d} x \mathrm{~d} t+\frac{1}{2 h_{\ell}} \iint_{\Omega \times\left[-h_{\ell}, 0\right]}\left|v-u_{o}\right|^{2} \mathrm{~d} x \mathrm{~d} t
\end{aligned}
$$

holds true for any comparison function $v \in L^{2}\left(\Omega_{T}, \mathbf{R}^{N}\right) \cap L^{p}\left(0, T ; W^{1, p}\left(\Omega, \mathbf{R}^{N}\right)\right)$ with $v(0) \in L^{2}\left(\Omega, \mathbf{R}^{N}\right)$.

3.5. Variational inequality for the limit map. In order to show that $u$ is a variational inequality to (3.2), we pass to the limit $\mathfrak{K} \ni \ell \rightarrow \infty$ in (3.11). As comparison map we choose $v \in L^{p}\left(0, T ; W^{1, p}\left(\Omega, \mathbf{R}^{N}\right)\right)$ with $\partial_{t} v \in L^{1}\left(0, T ; L^{2}\left(\Omega, \mathbf{R}^{N}\right)\right)$ and $v(0) \in L^{2}\left(\Omega, \mathbf{R}^{N}\right)$, which we extend to negative times $t \in\left(-h_{\ell}, 0\right]$ by $v(t)=v(0)$. By $(3.8)_{2}$ and lower semicontinuity we obtain that

$$
\begin{aligned}
& \iint_{\Omega_{T}}\left[f^{p}(x, D u)+g(x, u)\right] \mathrm{d} x \mathrm{~d} t \\
& \leq \liminf _{\mathfrak{K} \ni \ell \rightarrow \infty} \iint_{\Omega_{T}}\left[f^{p}\left(x, D u^{(\ell)}\right)+g\left(x, u^{(\ell)}\right)\right] \mathrm{d} x \mathrm{~d} t .
\end{aligned}
$$

Next, note that $\frac{1}{h_{\ell}}\left(v-v\left(t-h_{\ell}\right)\right) \rightarrow \partial_{t} v$ strongly in $L^{1}\left(0, T ; L^{2}\left(\Omega, \mathbf{R}^{N}\right)\right)$ as $\mathfrak{K} \ni \ell \rightarrow \infty$, since $\partial_{t} v \in L^{1}\left(0, T ; L^{2}\left(\Omega, \mathbf{R}^{N}\right)\right)$. Combining this with $(3.8)_{1}$, we have that

$$
\iint_{\Omega_{T}} \partial_{t} v \cdot(v-u) \mathrm{d} x \mathrm{~d} t=\lim _{\mathfrak{K} \ni \ell \rightarrow \infty} \frac{1}{h_{\ell}} \iint_{\Omega_{T}}\left(v-u^{(\ell)}\right) \cdot\left(v-v\left(t-h_{\ell}\right)\right) \mathrm{d} x \mathrm{~d} t .
$$

Since $v \in C^{0}\left([0, T] ; L^{2}\left(\Omega, \mathbf{R}^{N}\right)\right)$, we obtain

$$
\lim _{\ell \rightarrow \infty} \frac{1}{2 h_{\ell}} \iint_{\Omega \times\left[T-h_{\ell}, T\right]}|v-v(T)|^{2} \mathrm{~d} x \mathrm{~d} t=0 .
$$


This allows us to replace in the second last integral in (3.11) the function $v$ by its time slice $v(T)$. Moreover, since $u^{(\ell)}=u^{(\ell)}(T)$ on $\left(T-h_{\ell}, T\right]$ by definition, it remains to consider

$$
\lim _{\mathfrak{K} \ni \ell \rightarrow \infty} \int_{\Omega}\left|v(T)-u^{(\ell)}(T)\right|^{2} \mathrm{~d} x .
$$

To this end, observe that

$$
\begin{aligned}
\int_{\Omega} u(T) \cdot w \mathrm{~d} x & =\iint_{\Omega_{T}} \partial_{t} u \cdot w \mathrm{~d} x \mathrm{~d} t+\int_{\Omega} u_{o} \cdot w \mathrm{~d} x \\
& =\lim _{\ell \rightarrow \infty} \iint_{\Omega_{T}} \partial_{t} \tilde{u}^{(\ell)} \cdot w \mathrm{~d} x \mathrm{~d} t+\int_{\Omega} u_{o} \cdot w \mathrm{~d} x \\
& =\lim _{\ell \rightarrow \infty} \int_{\Omega} \tilde{u}^{(\ell)}(T) \cdot w \mathrm{~d} x .
\end{aligned}
$$

holds true for any $w \in L^{2}\left(\Omega, \mathbf{R}^{N}\right)$. Since $\tilde{u}^{(\ell)}(T)=u^{(\ell)}(T)$, this yields $u^{(\ell)}(T) \neg u(T)$ weakly in $L^{2}\left(\Omega, \mathbf{R}^{N}\right)$ as $\mathfrak{K} \ni \ell \rightarrow \infty$. Thus, by lower semicontinuity we conclude that

$$
\int_{\Omega}|v(T)-u(T)|^{2} \mathrm{~d} x \leq \liminf _{\mathfrak{K} \ni \ell \rightarrow \infty} \int_{\Omega}\left|v(T)-u^{(\ell)}(T)\right|^{2} \mathrm{~d} x .
$$

Finally, note that by $v(t)=v(0)$ for $t \in\left(-h_{\ell}, 0\right]$ we have that

$$
\frac{1}{2 h_{\ell}} \iint_{\Omega \times\left[-h_{\ell}, 0\right]}\left|v-u_{o}\right|^{2} \mathrm{~d} x \mathrm{~d} t=\frac{1}{2} \int_{\Omega}\left|v(0)-u_{o}\right|^{2} \mathrm{~d} x \mathrm{~d} t .
$$

Joining (3.12), (3.13), (3.14) and (3.15) we find after passing to the limit $\mathfrak{K} \ni \ell \rightarrow \infty$ that

$$
\begin{aligned}
& \iint_{\Omega_{T}}\left[f^{p}(x, D u)+g(x, u)\right] \mathrm{d} x \mathrm{~d} t \\
& \leq \iint_{\Omega_{T}}\left[f^{p}(x, D v)+g(x, v)\right] \mathrm{d} x \mathrm{~d} t+\iint_{\Omega_{T}} \partial_{t} v \cdot(v-u) \mathrm{d} x \mathrm{~d} t \\
& \quad-\frac{1}{2}\|(v-u)(T)\|_{L^{2}\left(\Omega, \mathbf{R}^{N}\right)}+\frac{1}{2}\left\|v(0)-u_{o}\right\|_{L^{2}\left(\Omega, \mathbf{R}^{N}\right)}
\end{aligned}
$$

holds true for any $v \in L^{p}\left(0, T ; W^{1, p}\left(\Omega, \mathbf{R}^{N}\right)\right)$ with $\partial_{t} v \in L^{1}\left(0, T ; L^{2}\left(\Omega, \mathbf{R}^{N}\right)\right)$ and $v(0) \in L^{2}\left(\Omega, \mathbf{R}^{N}\right)$. By means of Lemma B.1, this shows that $u$ is the required variational inequality to (3.2), which concludes the proof of Theorem 3.1.

\section{Existence of variational solutions for problems with superlinear growth: the case of $L^{2}$-initial data}

Theorem 4.1. Assume that initial values $u_{o} \in L^{2}\left(\Omega, \mathbf{R}^{N}\right)$ are given, that $f^{p}$ fulfills hypotheses (1.14) and (1.15) and $g$ satisfies (1.10) and (1.11). Then there exists a variational solution $u \in C^{0}\left([0, T] ; L^{2}\left(\Omega, \mathbf{R}^{N}\right)\right) \cap L^{p}\left(0, T ; W^{1, p}\left(\Omega, \mathbf{R}^{N}\right)\right)$ to (3.2) in the sense of Definition 1.3.

Proof. Let $\varepsilon>0$. We extend $u_{o}$ to $\mathbf{R}^{n}$ by zero and consider regularizations $u_{o, \varepsilon}:=u_{o} * \phi_{\varepsilon}$, where $\phi_{\varepsilon}$ denotes the standard mollifier. Note that $u_{o, \varepsilon} \in L^{2}\left(\Omega, \mathbf{R}^{N}\right) \cap$ $W^{1, p}\left(\Omega, \mathbf{R}^{N}\right)$. By Theorem 3.1 for each $\varepsilon \in(0,1)$ there exists a variational solution $u_{\varepsilon} \in C^{0}\left([0, T] ; L^{2}\left(\Omega, \mathbf{R}^{N}\right)\right) \cap L^{p}\left(0, T ; W^{1, p}\left(\Omega, \mathbf{R}^{N}\right)\right)$ to $(3.2)$ with initial datum $u_{o, \varepsilon}$, 
i.e. $u_{\varepsilon}$ satisfies the variational inequality

$$
\begin{aligned}
& \iint_{\Omega_{\tau}}\left[f^{p}\left(x, D u_{\varepsilon}\right)+g\left(x, u_{\varepsilon}\right)\right] \mathrm{d} x \mathrm{~d} t \\
& \leq \iint_{\Omega_{\tau}} \partial_{t} v \cdot\left(v-u_{\varepsilon}\right) \mathrm{d} x \mathrm{~d} t+\iint_{\Omega_{\tau}}\left[f^{p}(x, D v)+g(x, v)\right] \mathrm{d} x \mathrm{~d} t \\
& \quad-\frac{1}{2}\left\|\left(v-u_{\varepsilon}\right)(\tau)\right\|_{L^{2}\left(\Omega, \mathbf{R}^{N}\right)}^{2}+\frac{1}{2}\left\|v(0)-u_{o, \varepsilon}\right\|_{L^{2}\left(\Omega, \mathbf{R}^{N}\right)}^{2}
\end{aligned}
$$

for all $\tau \in[0, T]$ and any comparison map $v \in L^{p}\left(0, T ; W^{1, p}\left(\Omega, \mathbf{R}^{N}\right)\right)$ with $\partial_{t} v \in$ $L^{1}\left(0, T ; L^{2}\left(\Omega, \mathbf{R}^{N}\right)\right)$ and $v(0) \in L^{2}\left(\Omega, \mathbf{R}^{N}\right)$. From Lemma $2.15,(1.10)$ and (1.14) we infer that the energy bound

$$
\begin{aligned}
& \frac{1}{2} \sup _{t \in[0, T]}\left\|u_{\varepsilon}(t)\right\|_{L^{2}\left(\Omega, \mathbf{R}^{N}\right)}^{2}+\nu^{p} \iint_{\Omega_{T}}\left|D u_{\varepsilon}\right|^{p} \mathrm{~d} x \mathrm{~d} t \\
& \leq 2\left[L_{p}+M\right]\left|\Omega_{T}\right|+\left\|u_{o, \varepsilon}\right\|_{L^{2}\left(\Omega, \mathbf{R}^{N}\right)}^{2}
\end{aligned}
$$

holds true for any $\varepsilon \in(0,1)$. This implies that the sequence $\left(u_{\varepsilon}\right)_{\varepsilon \in(0,1)}$ is bounded in $L^{\infty}\left(0, T ; L^{2}\left(\Omega, \mathbf{R}^{N}\right)\right)$. If $p \leq 2$, we immediately know from $(4.2)$ that $\left(u_{\varepsilon}\right)_{\varepsilon \in(0,1)}$ is bounded in $L^{p}\left(0, T ; W^{1, p}\left(\Omega, \mathbf{R}^{N}\right)\right)$ as well. If $p>2$, we conclude from Poincaré's inequality and Hölder's inequality that

$$
\sup _{t \in[0, T]}\left\|u_{\varepsilon}(t)\right\|_{L^{p}\left(\Omega, \mathbf{R}^{N}\right)} \leq|\Omega|^{\frac{1}{p}-\frac{1}{2}} \sup _{t \in[0, T]}\left\|u_{\varepsilon}(t)\right\|_{L^{2}\left(\Omega, \mathbf{R}^{N}\right)}+c \sup _{t \in[0, T]}\left\|D u_{\varepsilon}\right\|_{L^{p}\left(\Omega, \mathbf{R}^{N}\right)},
$$

for a constant $c=c(n, p, \Omega)$. Since the right-hand side of the preceding inequality is bounded by $(4.2)$, the sequence $\left(u_{\varepsilon}\right)_{\varepsilon \in(0,1)}$ is bounded in $L^{p}\left(0, T ; W^{1, p}\left(\Omega, \mathbf{R}^{N}\right)\right)$. Hence, there exists a (not relabelled) subsequence and a limit map $u \in L^{\infty}\left(0, T ; L^{2}(\Omega\right.$, $\left.\left.\mathbf{R}^{N}\right)\right) \cap L^{p}\left(0, T ; W^{1, p}\left(\Omega, \mathbf{R}^{N}\right)\right)$, such that

$$
\begin{cases}u_{\varepsilon} \rightarrow & \text { weakly* in } L^{\infty}\left(0, T ; L^{2}\left(\Omega, \mathbf{R}^{N}\right)\right), \\ u_{\varepsilon} \neg u & \text { weakly in } L^{p}\left(0, T ; W^{1, p}\left(\Omega, \mathbf{R}^{N}\right)\right)\end{cases}
$$

as $\varepsilon \downarrow 0$. Next, we want to pass to the limit $\varepsilon \downarrow 0$ in (4.1). Since this is not directly possible because of the boundary term $\left\|\left(v-u_{\varepsilon}\right)(\tau)\right\|_{L^{2}\left(\Omega, \mathbf{R}^{N}\right)}^{2}$, we integrate (4.1) over $\tau \in\left[t_{o}, t_{o}+\delta\right]$ for some $\delta>0$ and $t_{o} \in[0, T-\delta]$ and divide the result by $\delta$. This yields

$$
\begin{aligned}
& \iint_{\Omega_{t_{o}}}\left[f^{p}\left(x, D u_{\varepsilon}\right)+g\left(x, u_{\varepsilon}\right)\right] \mathrm{d} x \mathrm{~d} t-\frac{1}{2} \int_{t_{o}}^{t_{o}+\delta}\left\|\left(v-u_{\varepsilon}\right)(\tau)\right\|_{L^{2}\left(\Omega, \mathbf{R}^{N}\right)}^{2} \mathrm{~d} \tau \\
& \leq \int_{t_{o}}^{t_{o}+\delta} \iint_{\Omega_{\tau}} \partial_{t} v \cdot\left(v-u_{\varepsilon}\right) \mathrm{d} x \mathrm{~d} t \mathrm{~d} \tau+\iint_{\Omega_{t_{o}+\delta}}\left[f^{p}(x, D v)+g(x, v)\right] \mathrm{d} x \mathrm{~d} t \\
& \quad+\frac{1}{2}\left\|v(0)-u_{o, \varepsilon}\right\|_{L^{2}\left(\Omega, \mathbf{R}^{N}\right)}^{2} .
\end{aligned}
$$

By $u_{\varepsilon} \rightarrow u$ weakly in $L^{2}\left(\Omega_{T}, \mathbf{R}^{N}\right),(4.3)_{2},(1.14),(1.15)$ and (1.10) we can use the lower semicontinuity of the the left-hand side in order to pass to the limit. Next, since $u_{o, \varepsilon} \rightarrow u_{o}$ in $L^{2}\left(\Omega, \mathbf{R}^{N}\right)$, we conclude that

$$
\lim _{\varepsilon \downarrow 0}\left\|v(0)-u_{o, \varepsilon}\right\|_{L^{2}\left(\Omega, \mathbf{R}^{N}\right)}^{2}=\left\|v(0)-u_{o}\right\|_{L^{2}\left(\Omega, \mathbf{R}^{N}\right)}^{2} .
$$

Further, by $(4.3)_{1}$ and $\partial_{t} v \in L^{1}\left(0, T ; L^{2}\left(\Omega, \mathbf{R}^{N}\right)\right)$ we have that

$$
\lim _{\varepsilon \downarrow 0} \iint_{\Omega_{\tau}} \partial_{t} v \cdot\left(v-u_{\varepsilon}\right) \mathrm{d} x \mathrm{~d} t=\iint_{\Omega_{\tau}} \partial_{t} v \cdot(v-u) \mathrm{d} x \mathrm{~d} t .
$$


Since

$$
\sup _{\varepsilon \in(0,1)} \sup _{\tau \in[0, T]} \iint_{\Omega_{\tau}} \partial_{t} v \cdot\left(v-u_{\varepsilon}\right) \mathrm{d} x \mathrm{~d} t \leq\left\|\partial_{t} v\right\|_{L^{1}\left(0, T ; L^{2}\left(\Omega, \mathbf{R}^{N}\right)\right)}\left\|v-u_{\varepsilon}\right\|_{L^{\infty}\left(0, T ; L^{2}\left(\Omega, \mathbf{R}^{N}\right)\right)},
$$

which is finite by $\partial_{t} v \in L^{1}\left(0, T ; L^{2}\left(\Omega, \mathbf{R}^{N}\right)\right)$ and $(4.3)_{1}$, we may apply the dominated convergence theorem. Thus, combining the preceding computations and passing to the limit $\varepsilon \downarrow 0$ in (4.4), we obtain that

$$
\begin{aligned}
& \iint_{\Omega_{t_{o}}}\left[f^{p}(x, D u)+g(x, u)\right] \mathrm{d} x \mathrm{~d} t-\frac{1}{2} \int_{t_{o}}^{t_{o}+\delta}\|(v-u)(\tau)\|_{L^{2}\left(\Omega, \mathbf{R}^{N}\right)}^{2} \mathrm{~d} \tau \\
& \leq \int_{t_{o}}^{t_{o}+\delta} \iint_{\Omega_{\tau}} \partial_{t} v \cdot(v-u) \mathrm{d} x \mathrm{~d} t \mathrm{~d} \tau+\iint_{\Omega_{t_{o}+\delta}}\left[f^{p}(x, D v)+g(x, v)\right] \mathrm{d} x \mathrm{~d} t \\
& \quad+\frac{1}{2}\left\|v(0)-u_{o}\right\|_{L^{2}\left(\Omega, \mathbf{R}^{N}\right)}^{2} .
\end{aligned}
$$

Here, we pass to the limit $\delta \downarrow 0$ and infer that

$$
\begin{aligned}
& \iint_{\Omega_{t_{o}}}\left[f^{p}(x, D u)+g(x, u)\right] \mathrm{d} x \mathrm{~d} t \\
& \leq \iint_{\Omega_{t_{o}}} \partial_{t} v \cdot(v-u) \mathrm{d} x \mathrm{~d} t+\iint_{\Omega_{t_{o}}}\left[f^{p}(x, D v)+g(x, v)\right] \mathrm{d} x \mathrm{~d} t \\
& \quad-\frac{1}{2}\|(v-u)(\tau)\|_{L^{2}\left(\Omega, \mathbf{R}^{N}\right)}^{2}+\frac{1}{2}\left\|v(0)-u_{o}\right\|_{L^{2}\left(\Omega, \mathbf{R}^{N}\right)}^{2}
\end{aligned}
$$

for any $t_{o} \in[0, T]$ and any comparison map $v \in L^{p}\left(0, T ; W^{1, p}\left(\Omega, \mathbf{R}^{N}\right)\right)$ with $\partial_{t} v \in$ $L^{1}\left(0, T ; L^{2}\left(\Omega, \mathbf{R}^{N}\right)\right)$ and $v(0) \in L^{2}\left(\Omega, \mathbf{R}^{N}\right)$. Since $u \in C^{0}\left([0, T] ; L^{2}\left(\Omega, \mathbf{R}^{N}\right)\right)$ by Lemma A.1, it is the desired variational solution to (3.2) in the sense of Definition 1.3.

\section{Proof of the main result}

We consider a sequence $\left(p_{i}\right)_{i \in \mathbf{N}}$ with $p_{i}>1$ for any $i \in \mathbf{N}$ and $p_{i} \downarrow 1$ as $i \rightarrow \infty$. Without loss of generality let $p_{i} \leq 2$ for every $i \in \mathbf{N}$. We assume that the Cauchy data $u_{i, o}$ satisfy (1.16) with exponent $p_{i}$. For each $i \in \mathbf{N}$ the mapping

$$
u_{i} \in C^{0}\left([0, T] ; L^{2}\left(\Omega, \mathbf{R}^{N}\right)\right) \cap L^{p_{i}}\left(0, T ; W^{1, p_{i}}\left(\Omega, \mathbf{R}^{N}\right)\right)
$$

denotes the unique variational solution to (1.4) with exponent $p_{i}$ and Cauchy datum $u_{i, o}$ in the sense of Definition 1.3. From Lemma 2.15 and the growth conditions (1.14) and (1.10) we infer the energy bound

$$
\frac{1}{2} \sup _{t \in[0, T]}\left\|u_{i}(t)\right\|_{L^{2}\left(\Omega, \mathbf{R}^{N}\right)}^{2}+\nu^{p_{i}} \iint_{\Omega_{T}}\left|D u_{i}\right|^{p_{i}} \mathrm{~d} x \mathrm{~d} t \leq 2\left[L_{p_{i}}+M\right]\left|\Omega_{T}\right|+\left\|u_{i, o}\right\|_{L^{2}\left(\Omega, \mathbf{R}^{N}\right)}^{2} .
$$

By means of (1.18) and Hölder's inequality we therefore conclude that

$$
\left(u_{i}\right)_{i \in \mathbf{N}} \text { is bounded in } L^{\infty}\left(0, T ; L^{2}\left(\Omega, \mathbf{R}^{N}\right)\right) \cap L^{1}\left(0, T ; W^{1,1}\left(\Omega, \mathbf{R}^{N}\right)\right) .
$$

Thus, there exists a (not relabelled) subsequence and a map $u \in L^{\infty}\left(0, T ; L^{2}\left(\Omega, \mathbf{R}^{N}\right)\right)$ such that

$$
u_{i} \stackrel{*}{\rightarrow} u \quad \text { weakly* in } L^{\infty}\left(0, T ; L^{2}\left(\Omega, \mathbf{R}^{N}\right)\right) .
$$


By Lemma 2.2 we have that $u \in L_{w *}^{1}\left(0, T ; \operatorname{BV}\left(\Omega, \mathbf{R}^{N}\right)\right)$. Next, Lemma 2.16 yields that

$$
\begin{aligned}
& \int_{0}^{T-h}\left\|u_{i}(t+h)-u_{i}(t)\right\|_{W^{-\ell, 2}\left(\Omega, \mathbf{R}^{N}\right)} \mathrm{d} t \\
& \leq c\left[1+\left\|D u_{i}\right\|_{L^{p_{i}\left(\Omega_{T}, \mathbf{R}^{N}\right)}}^{p_{i}-1}+\left\|u_{i}\right\|_{L^{2}\left(\Omega_{T}, \mathbf{R}^{N}\right)}\right] \sqrt{h}
\end{aligned}
$$

holds true for any $\ell \geq 1$ and $h \in(0,1)$ with a constant $c=c(n, N, L, M, \ell,|\Omega|, T)$. For $\ell \geq \frac{n}{2}$ we infer from Lemma 2.1 with the choice

$$
(X, B, Y, p)=\left(W^{1,1}\left(\Omega, \mathbf{R}^{N}\right), L^{1}\left(\Omega, \mathbf{R}^{N}\right), W^{-\ell, 2}\left(\Omega, \mathbf{R}^{N}\right), 1\right)
$$

that there exists a further (not relabelled) subsequence such that

$$
u_{i} \rightarrow u \text { in } L^{1}\left(\Omega_{T}, \mathbf{R}^{N}\right) \text { and a.e. in } \Omega_{T} \text { as } i \rightarrow \infty .
$$

Together with (5.1) this implies that $D u_{i} \stackrel{*}{\rightarrow} D u$ weakly* in $\operatorname{RM}\left(\Omega, \mathbf{R}^{N \times n}\right)$ as $i \rightarrow \infty$. Since any function $w \in L^{2}\left(0, T ; W^{1,2}\left(\Omega, \mathbf{R}^{N}\right)\right)$ with $\partial_{t} w \in L^{1}\left(0, T ; L^{2}\left(\Omega, \mathbf{R}^{N}\right)\right)$ and $\left.w(0) \in L^{2}\left(\Omega, \mathbf{R}^{N}\right)\right)$ is an admissible comparison map in (1.17) for any $p_{i}, i \in \mathbf{N}$, we obtain variational inequalities

$$
\begin{aligned}
& \iint_{\Omega_{\tau}}\left[f^{p_{i}}\left(x, D u_{i}\right)+g\left(x, u_{i}\right)\right] \mathrm{d} x \mathrm{~d} t \\
& \leq \iint_{\Omega_{\tau}} \partial_{t} w \cdot\left(w-u_{i}\right) \mathrm{d} x \mathrm{~d} t+\iint_{\Omega_{\tau}}\left[f^{p_{i}}(x, D w)+g(x, w)\right] \mathrm{d} x \mathrm{~d} t \\
& \quad-\frac{1}{2}\left\|\left(w-u_{i}\right)(\tau)\right\|_{L^{2}\left(\Omega, \mathbf{R}^{N}\right)}^{2}+\frac{1}{2}\left\|w(0)-u_{i, o}\right\|_{L^{2}\left(\Omega, \mathbf{R}^{N}\right)}^{2}
\end{aligned}
$$

for any $\tau \in[0, T]$. Our next goal is to pass to the limit $i \rightarrow \infty$ in the preceding inequality. Integrating over $\tau \in\left(t_{o}, t_{o}+\delta\right)$ for some $\delta \in(0, T), t_{o} \in[0, T-\delta]$ and dividing the result by $\delta$ leads to

$$
\begin{aligned}
& \iint_{\Omega_{t_{o}}} f^{p_{i}}\left(x, D u_{i}\right) \mathrm{d} x \mathrm{~d} t \\
& \leq \int_{t_{o}}^{t_{o}+\delta} \iint_{\Omega_{\tau}} \partial_{t} w \cdot\left(w-u_{i}\right) \mathrm{d} x \mathrm{~d} t \mathrm{~d} \tau+\iint_{\Omega_{t_{o}+\delta}} f^{p_{i}}(x, D w) \mathrm{d} x \mathrm{~d} t \\
& \quad+\iint_{\Omega_{t_{o}+\delta}} g(x, w) \mathrm{d} x \mathrm{~d} t-\iint_{\Omega_{t_{o}}} g\left(x, u_{i}\right) \mathrm{d} x \mathrm{~d} t \\
& \quad-\frac{1}{2} f_{t_{o}}^{t_{o}+\delta}\left\|\left(w-u_{i}\right)(\tau)\right\|_{L^{2}\left(\Omega, \mathbf{R}^{N}\right)}^{2} \mathrm{~d} \tau+\frac{1}{2}\left\|w(0)-u_{i, o}\right\|_{L^{2}\left(\Omega, \mathbf{R}^{N}\right)}^{2} .
\end{aligned}
$$

By Reshetnyak's lower semicontinuity theorem, i.e. Theorem 2.3, Fatou's lemma and Hölder's inequality we infer that

$$
\begin{aligned}
\int_{0}^{t_{o}} \mathcal{F}[u(t)] \mathrm{d} t & \leq \liminf _{i \rightarrow \infty} \int_{0}^{t_{o}} \mathcal{F}\left[u_{i}\right] \mathrm{d} t=\liminf _{i \rightarrow \infty} \iint_{\Omega_{t_{o}}} f\left(x, D u_{i}\right) \mathrm{d} x \mathrm{~d} t \\
& \leq \liminf _{i \rightarrow \infty}\left[\iint_{\Omega_{t_{o}}} f^{p_{i}}\left(x, D u_{i}\right) \mathrm{d} x \mathrm{~d} t\right]^{\frac{1}{p_{i}}} .
\end{aligned}
$$


Combining this with the second last inequality, we conclude that

$$
\begin{aligned}
\int_{0}^{t_{o}} \mathcal{F}[u(t)] \mathrm{d} t \leq & \liminf _{i \rightarrow \infty}\left[f_{t_{o}}^{t_{o}+\delta} \iint_{\Omega_{\tau}} \partial_{t} w \cdot\left(w-u_{i}\right) \mathrm{d} x \mathrm{~d} t \mathrm{~d} \tau\right. \\
& +\iint_{\Omega_{t_{o}+\delta}} f^{p_{i}}(x, D w) \mathrm{d} x \mathrm{~d} t \\
& +\iint_{\Omega_{t_{o}+\delta}} g(x, w) \mathrm{d} x \mathrm{~d} t-\iint_{\Omega_{t_{o}}} g\left(x, u_{i}\right) \mathrm{d} x \mathrm{~d} t \\
& \left.-\frac{1}{2} f_{t_{o}}^{t_{o}+\delta}\left\|\left(w-u_{i}\right)(\tau)\right\|_{L^{2}\left(\Omega, \mathbf{R}^{N}\right)}^{2} \mathrm{~d} \tau+\frac{1}{2}\left\|w(0)-u_{i, o}\right\|_{L^{2}\left(\Omega, \mathbf{R}^{N}\right)}^{2}\right]^{\frac{1}{p_{i}}} .
\end{aligned}
$$

At this stage, we consider the terms appearing on the right-hand side of the preceding inequality separately. By $\partial_{t} w \in L^{1}\left(0, T ; L^{2}\left(\Omega, \mathbf{R}^{N}\right)\right)$, (5.2) and the dominated convergence theorem, we have that

$$
\lim _{i \rightarrow \infty} \int_{t_{o}}^{t_{o}+\delta} \iint_{\Omega_{\tau}} \partial_{t} w \cdot\left(w-u_{i}\right) \mathrm{d} x \mathrm{~d} t \mathrm{~d} \tau=f_{t_{o}}^{t_{o}+\delta} \iint_{\Omega_{\tau}} \partial_{t} w \cdot(w-u) \mathrm{d} x \mathrm{~d} t \mathrm{~d} \tau .
$$

Next, dominated convergence yields

$$
\lim _{i \rightarrow \infty} \iint_{\Omega_{t_{o}+\delta}} f^{p_{i}}(x, D w) \mathrm{d} x \mathrm{~d} t=\iint_{\Omega_{t_{o}+\delta}} f(x, D w) \mathrm{d} x \mathrm{~d} t .
$$

By (5.3) and Fatou's lemma, which we may apply by (1.10), we have that

$$
\iint_{\Omega_{t_{o}}} g(x, u) \mathrm{d} x=\iint_{\Omega_{t_{o}}} \lim _{i \rightarrow \infty} g\left(x, u_{i}\right) \mathrm{d} x \mathrm{~d} t \leq \liminf _{i \rightarrow \infty} \iint_{\Omega_{t_{o}}} g\left(x, u_{i}\right) \mathrm{d} x \mathrm{~d} t .
$$

Moreover, by (5.2) and lower semicontinuity we infer that

$$
\frac{1}{2} f_{t_{o}}^{t_{o}+\delta}\|(w-u)(\tau)\|_{L^{2}\left(\Omega, \mathbf{R}^{N}\right)}^{2} \mathrm{~d} \tau \leq \liminf _{i \rightarrow \infty} \frac{1}{2} f_{t_{o}}^{t_{o}+\delta}\left\|\left(w-u_{i}\right)(\tau)\right\|_{L^{2}\left(\Omega, \mathbf{R}^{N}\right)}^{2} \mathrm{~d} \tau .
$$

Finally, by assumption (1.18) we conclude that

$$
\lim _{i \rightarrow \infty} \frac{1}{2}\left\|w(0)-u_{i, o}\right\|_{L^{2}\left(\Omega, \mathbf{R}^{N}\right)}^{2}=\frac{1}{2}\left\|w(0)-u_{o}\right\|_{L^{2}\left(\Omega, \mathbf{R}^{N}\right)}^{2} .
$$

Inserting (5.5), (5.6), (5.7), (5.8) and (5.9) into (5.4) implies that

$$
\begin{aligned}
& \int_{0}^{t_{o}} \mathcal{F}[u(t)] \mathrm{d} t+\iint_{\Omega_{t_{o}}} g(x, u) \mathrm{d} x \mathrm{~d} t \\
& \leq \int_{t_{o}}^{t_{o}+\delta} \iint_{\Omega_{\tau}} \partial_{t} w \cdot(w-u) \mathrm{d} x \mathrm{~d} t \mathrm{~d} \tau+\iint_{\Omega_{t_{o}+\delta}}[f(x, D w)+g(x, w)] \mathrm{d} x \mathrm{~d} t \\
& \quad-\frac{1}{2} \int_{t_{o}}^{t_{o}+\delta}\|(w-u)(\tau)\|_{L^{2}\left(\Omega, \mathbf{R}^{N}\right)}^{2} \mathrm{~d} \tau+\frac{1}{2}\left\|w(0)-u_{o}\right\|_{L^{2}\left(\Omega, \mathbf{R}^{N}\right)}^{2}
\end{aligned}
$$

holds true for any $w \in L^{2}\left(0, T ; W^{1,2}\left(\Omega, \mathbf{R}^{N}\right)\right)$ with $\partial_{t} w \in L^{1}\left(0, T ; L^{2}\left(\Omega, \mathbf{R}^{N}\right)\right)$ and $\left.w(0) \in L^{2}\left(\Omega, \mathbf{R}^{N}\right)\right)$.

Next, we need to show that (5.10) holds true for general comparison maps $v \in$ $L_{w *}^{1}\left(0, T ; \operatorname{BV}\left(\Omega, \mathbf{R}^{N}\right)\right)$ with $\partial_{t} v \in L^{1}\left(0, T ; L^{2}\left(\Omega, \mathbf{R}^{N}\right)\right)$ and $v(0) \in L^{2}\left(\Omega, \mathbf{R}^{N}\right)$ as well. To this end, using the extension operator from Lemma 2.5, we extend $v$ to $\mathbf{R}^{n}$ and then consider regularizations $v_{\varepsilon}:=v * \phi_{\varepsilon}$ of the extensions, where $\phi_{\varepsilon}$ denotes the standard mollifier in $\mathbf{R}^{n}$. By Lemma 2.6 (ii) $w:=v_{\varepsilon}$ is an admissible comparison 
map in (5.10). In the following, we compute the limit $\varepsilon \downarrow 0$ of the appearing terms separately. First, we have that

$$
\begin{aligned}
& \left|\iint_{\Omega_{\tau}} \partial_{t} v_{\varepsilon} \cdot\left(v_{\varepsilon}-u\right)-\partial_{t} v \cdot(v-u) \mathrm{d} x \mathrm{~d} t\right| \\
& \quad \leq\left\|\partial_{t} v_{\varepsilon}-\partial_{t} v\right\|_{L^{1}\left(0, T ; L^{2}\left(\Omega, \mathbf{R}^{N}\right)\right)}\left\|v_{\varepsilon}-u\right\|_{L^{\infty}\left(0, T ; L^{2}\left(\Omega, \mathbf{R}^{N}\right)\right)} \\
& \quad+\int_{0}^{\tau}\left\|\partial_{t} v\right\|_{L^{2}\left(\Omega, \mathbf{R}^{N}\right)}\left\|v_{\varepsilon}-v\right\|_{L^{2}\left(\Omega, \mathbf{R}^{N}\right)} \mathrm{d} t .
\end{aligned}
$$

Observe that the first term on the right-hand side of the preceding inequality vanishes in the limit $\varepsilon \downarrow 0$ by Lemma 2.6 (iv). Now, we turn our attention to the second integral. We know that $v(t) \in L^{2}\left(\Omega, \mathbf{R}^{N}\right)$ for all $t \in[0, T]$, since $v \in C^{0}\left(0, T ; L^{2}\left(\Omega, \mathbf{R}^{N}\right)\right)$. Therefore, for any $t \in[0, T]$ we have that $v_{\varepsilon}(t) \rightarrow v(t)$ in $L^{2}\left(\Omega, \mathbf{R}^{N}\right)$ as $\varepsilon \downarrow 0$. Hence, for a.e. $t \in[0, T]$ there holds

$$
\lim _{\varepsilon \downarrow 0}\left\|\partial_{t} v(t)\right\|_{L^{2}\left(\Omega, \mathbf{R}^{N}\right)}\left\|v_{\varepsilon}(t)-v(t)\right\|_{L^{2}\left(\Omega, \mathbf{R}^{N}\right)}=0 .
$$

Furthermore, by Lemma 2.6 (iv) we know that

$$
\begin{aligned}
& \sup _{\varepsilon \in(0,1)}\left\|\partial_{t} v\right\|_{L^{2}\left(\Omega, \mathbf{R}^{N}\right)}\left\|v_{\varepsilon}-v\right\|_{L^{2}\left(\Omega, \mathbf{R}^{N}\right)} \\
& \leq 2\left\|\partial_{t} v\right\|_{L^{2}\left(\mathbf{R}^{n}, \mathbf{R}^{N}\right)} \sup _{t \in[0, T]}\|v(t)\|_{L^{2}\left(\mathbf{R}^{n}, \mathbf{R}^{N}\right)} \in L^{1}([0, T]) .
\end{aligned}
$$

Therefore, by the dominated convergence theorem, also the second term vanishes in the limit $\varepsilon \downarrow 0$. Hence, another application of the dominated convergence theorem yields

$$
\lim _{\varepsilon \downarrow 0} \int_{t_{o}}^{t_{o}+\delta} \iint_{\Omega_{\tau}} \partial_{t} v_{\varepsilon} \cdot\left(v_{\varepsilon}-u\right) \mathrm{d} x \mathrm{~d} t=\int_{t_{o}}^{t_{o}+\delta} \iint_{\Omega_{\tau}} \partial_{t} v \cdot(v-u) \mathrm{d} x \mathrm{~d} t \mathrm{~d} \tau .
$$

Next, since $|D v(t)|(\partial \Omega)=0$ for a.e. $t \in[0, T]$ by Lemma 2.5 (i), we have that

$$
\mathcal{F}[v(t)]=\int_{\Omega} f(x, \nabla v(t)) \mathrm{d} x+\int_{\bar{\Omega}} f^{\infty}\left(x, \frac{D^{s} v}{\left|D^{s} v\right|}\right) \mathrm{d}\left|D^{s} v\right| .
$$

Therefore, from Lemma $2.6(\mathrm{v})$ and Reshetnyak's continuity theorem, i.e. Theorem 2.4, we infer that

$$
\lim _{\varepsilon \downarrow 0} \int_{\Omega} f\left(x, D v_{\varepsilon}(t)\right) \mathrm{d} x=\mathcal{F}[v(t)]
$$

for a.e. $t \in[0, T]$. Furthermore, for any $\varepsilon \in(0,1)$ we have that

$$
\mathcal{F}\left[v_{\varepsilon}(t)\right]=\int_{\Omega} f\left(x, D v_{\varepsilon}(t)\right) \mathrm{d} x \leq L \int_{\Omega}\left(1+\left|D v_{\varepsilon}(t)\right|\right) \mathrm{d} x \leq L\left(|\Omega|+|D v(t)|\left(\mathbf{R}^{n}\right)\right) .
$$

Thus, by the dominated convergence theorem, we find that

$$
\lim _{\varepsilon \downarrow 0} \iint_{\Omega_{t_{o}+\delta}} f\left(x, D v_{\varepsilon}\right) \mathrm{d} x \mathrm{~d} t=\int_{0}^{t_{o}+\delta} \mathcal{F}[v(t)] \mathrm{d} t .
$$

Since $v_{\varepsilon} \rightarrow v$ in $L^{2}\left(\Omega_{T}, \mathbf{R}^{N}\right)$ by Lemma 2.6 (i), we deduce from Lemma 2.10 with $m=N$ and $p_{o}=p=2$ that

$$
\lim _{\varepsilon \downarrow 0} \iint_{\Omega_{t_{o}+\delta}} g\left(x, v_{\varepsilon}\right) \mathrm{d} x \mathrm{~d} t=\iint_{\Omega_{t_{o}+\delta}} g(x, v) \mathrm{d} x \mathrm{~d} t .
$$


Finally, by Lemma 2.6 (i), we conclude that

$$
\lim _{\varepsilon \downarrow 0} \int_{t_{o}}^{t_{o}+\delta}\left\|\left(v_{\varepsilon}-u\right)(\tau)\right\|_{L^{2}\left(\Omega, \mathbf{R}^{N}\right)}^{2} \mathrm{~d} \tau=f_{t_{o}}^{t_{o}+\delta}\|(v-u)(\tau)\|_{L^{2}\left(\Omega, \mathbf{R}^{N}\right)}^{2} \mathrm{~d} \tau
$$

and by $v(0) \in L^{2}\left(\Omega, \mathbf{R}^{N}\right)$ that

$$
\lim _{\varepsilon \downarrow 0}\left\|v_{\varepsilon}(0)-u_{o}\right\|_{L^{2}\left(\Omega, \mathbf{R}^{N}\right)}^{2}=\left\|v(0)-u_{o}\right\|_{L^{2}\left(\Omega, \mathbf{R}^{N}\right)}^{2}
$$

Hence, passing to the limit $\varepsilon \downarrow 0$ in (5.10) with the choice $w=v_{\varepsilon}$, by (5.11), (5.12), (5.13), (5.14) and (5.15) we infer that

$$
\begin{aligned}
& \int_{0}^{t_{o}} \mathcal{F}[u(t)] \mathrm{d} t+\iint_{\Omega_{t_{o}}} g(x, v) \mathrm{d} x \mathrm{~d} t \\
& \leq f_{t_{o}}^{t_{o}+\delta} \iint_{\Omega_{\tau}} \partial_{t} v \cdot(v-u) \mathrm{d} x \mathrm{~d} t \mathrm{~d} \tau+\int_{0}^{t_{o}+\delta} \mathcal{F}[v(t)] \mathrm{d} t+\iint_{\Omega_{t_{o}+\delta}} g(x, v) \mathrm{d} x \mathrm{~d} t \\
& \quad-\frac{1}{2} f_{t_{o}}^{t_{o}+\delta}\|(v-u)(\tau)\|_{L^{2}\left(\Omega, \mathbf{R}^{N}\right)}^{2} \mathrm{~d} \tau+\frac{1}{2}\left\|v(0)-u_{o}\right\|_{L^{2}\left(\Omega, \mathbf{R}^{N}\right)}^{2}
\end{aligned}
$$

for any function $v \in L_{w *}^{1}\left(0, T ; \operatorname{BV}\left(\Omega, \mathbf{R}^{N}\right)\right)$ with $\partial_{t} v \in L^{1}\left(0, T ; L^{2}\left(\Omega, \mathbf{R}^{N}\right)\right)$ and $v(0) \in$ $\left.L^{2}\left(\Omega, \mathbf{R}^{N}\right)\right)$. Finally, we let $\delta \downarrow 0$ in the preceding inequality, which yields

$$
\begin{aligned}
& \int_{0}^{t_{o}} \mathcal{F}[u] \mathrm{d} t+\iint_{\Omega_{t_{o}}} g(x, v) \mathrm{d} x \mathrm{~d} t \\
& \leq \iint_{\Omega_{t_{o}}} \partial_{t} v \cdot(v-u) \mathrm{d} x \mathrm{~d} t+\int_{0}^{t_{o}} \mathcal{F}[v(t)] \mathrm{d} t+\iint_{\Omega_{t_{o}}} g(x, v) \mathrm{d} x \mathrm{~d} t \\
& \quad-\frac{1}{2}\left\|(v-u)\left(t_{o}\right)\right\|_{L^{2}\left(\Omega, \mathbf{R}^{N}\right)}^{2}+\frac{1}{2}\left\|v(0)-u_{o}\right\|_{L^{2}\left(\Omega, \mathbf{R}^{N}\right)}^{2}
\end{aligned}
$$

for a.e. $t_{o} \in[0, T]$. Hence, $u$ is a variational solution to (1.3) in the sense of Definition 1.1. Therefore, by means of Lemma A.3 we find that $u \in C^{0}\left([0, T] ; L^{2}\left(\Omega, \mathbf{R}^{N}\right)\right)$ and by Lemma A.5 $u$ is unique. As a consequence, we have that the convergence assertions (5.2), (5.3) and $D u_{i} \rightarrow D u$ weakly* in $\operatorname{RM}\left(\Omega, \mathbf{R}^{N \times n}\right)$ actually hold true for the whole sequence $\left(u_{i}\right)_{i \in \mathbf{N}}$. This concludes the proof of Theorem 1.5.

\section{Appendix A. Continuity and uniqueness}

In Definition 1.3 we require variational solutions $u$ to be in $C^{0}\left([0, T] ; L^{2}\left(\Omega, \mathbf{R}^{N}\right)\right)$. However, the following lemma shows that any map $u \in L^{\infty}\left(0, T ; L^{2}\left(\Omega, \mathbf{R}^{N}\right)\right)$ satisfying (1.17) is a variational solution in the sense of Definition 1.3. Its proof is similar to the proof of [15, Lemma 3.2].

Lemma A.1. Suppose that $u_{p, o}$ fulfills (1.16) and that $u \in L^{\infty}\left(0, T ; L^{2}\left(\Omega, \mathbf{R}^{N}\right)\right) \cap$ $L^{p}\left(0, T ; W^{1, p}\left(\Omega, \mathbf{R}^{N}\right)\right)$ satisfies the variational inequality (1.17) with exponent $p$ and initial datum $u_{p, o}$ for a.e. $\tau \in[0, T]$ and any map $v \in L^{p}\left(0, T ; W^{1, p}\left(\Omega, \mathbf{R}^{N}\right)\right)$ with $\partial_{t} v \in L^{1}\left(0, T ; L^{2}\left(\Omega, \mathbf{R}^{N}\right)\right)$ and $v(0) \in L^{2}\left(\Omega, \mathbf{R}^{N}\right)$. Then, $u \in C^{0}\left([0, T] ; L^{2}\left(\Omega, \mathbf{R}^{N}\right)\right)$.

Proof. Let $h>0$ and $\varepsilon=\varepsilon(h):=h^{\frac{1}{2(n+1) p}}$. We consider the mollification $\llbracket u \rrbracket_{h}$ defined according to $(2.2)$ with initial values $u_{p, o, h}:=u_{p, o} * \phi_{\varepsilon(h)}$, where $u_{p, o}$ is extended to $\mathbf{R}^{n}$ by zero and $\phi_{\varepsilon}$ denotes the standard mollifier in $\mathbf{R}^{n}$. By Lemma 2.7 we have that $\llbracket u \rrbracket_{h} \in L^{p}\left(0, T ; W^{1, p}\left(\Omega, \mathbf{R}^{N}\right)\right)$ with initial values $\llbracket u \rrbracket_{h}(0)=u_{p, o, h} \in L^{2}\left(\Omega, \mathbf{R}^{N}\right)$. 
Moreover, $\partial_{t} \llbracket u \rrbracket_{h} \in L^{1}\left(0, T ; L^{2}\left(\Omega, \mathbf{R}^{N}\right)\right)$ by (2.3). Noting that by (2.3) we have

$$
\iint_{\Omega_{\tau}} \partial_{t} \llbracket u \rrbracket_{h} \cdot\left(\llbracket u \rrbracket_{h}-u\right) \mathrm{d} x \mathrm{~d} t=-\frac{1}{h} \iint_{\Omega_{\tau}}\left|\llbracket u \rrbracket_{h}-u\right|^{2} \mathrm{~d} x \mathrm{~d} t \leq 0,
$$

by (1.17) with the choice $\llbracket u \rrbracket_{h}$ as comparison map, we find that

$$
\begin{aligned}
\frac{1}{2}\left\|\left(\llbracket u \rrbracket_{h}-u\right)(\tau)\right\|_{L^{2}\left(\Omega, \mathbf{R}^{N}\right)}^{2} \leq & \iint_{\Omega_{\tau}} f^{p}\left(x, D \llbracket u \rrbracket_{h}\right)-f^{p}(x, D u) \mathrm{d} x \mathrm{~d} t \\
& +\iint_{\Omega_{\tau}} g\left(x, \llbracket u \rrbracket_{h}\right)-g(x, u) \mathrm{d} x \mathrm{~d} t \\
& +\frac{1}{2}\left\|u_{p, o, h}-u_{p, o}\right\|_{L^{2}\left(\Omega, \mathbf{R}^{N}\right)}^{2}
\end{aligned}
$$

for a.e. $\tau \in[0, T]$. Taking the supremum over $\tau \in[0, T]$ and using Lemma 2.10 for $f$ with $m=N n, p_{o}=p$ and for $g$ with $m=N, p=p_{o}=2$, we obtain that

$$
\begin{aligned}
& \frac{1}{2} \sup _{\tau \in[0, T]}\left\|\left(\llbracket u \rrbracket_{h}-u\right)(\tau)\right\|_{L^{2}\left(\Omega, \mathbf{R}^{N}\right)}^{2} \\
& \leq c(N n, p) L_{p} \iint_{\Omega_{T}}\left(1+\left|D \llbracket u \rrbracket_{h}\right|^{p-1}+|D u|^{p-1}\right)\left|D \llbracket u \rrbracket_{h}-D u\right| \mathrm{d} x \mathrm{~d} t \\
& \quad+c(N) M \iint_{\Omega_{T}}\left(1+\left|\llbracket u \rrbracket_{h}\right|+|u|\right)\left|\llbracket u \rrbracket_{h}-u\right| \mathrm{d} x \mathrm{~d} t+\frac{1}{2}\left\|u_{p, o, h}-u_{p, o}\right\|_{L^{2}\left(\Omega, \mathbf{R}^{N}\right)}^{2} .
\end{aligned}
$$

In the last inequality we pass to the limit $h \downarrow 0$, taking into account that $u_{p, o, h} \rightarrow u_{p, o}$ in $L^{2}\left(\Omega, \mathbf{R}^{N}\right)$, that $D \llbracket u \rrbracket_{h} \rightarrow D u$ in $L^{p}\left(\Omega_{T}, \mathbf{R}^{N n}\right)$ by Lemma 2.13 , and that $\llbracket u \rrbracket_{h} \rightarrow u$ in $L^{2}\left(\Omega_{T}, \mathbf{R}^{N}\right)$ by Lemma 2.7. The latter holds true, since on the one hand the time regularization $\llbracket u \rrbracket_{h}^{u_{p, o}}$ of $u$ with initial value $u_{p, o}$ strongly converges in $L^{2}\left(\Omega_{T}, \mathbf{R}^{N}\right)$, while on the other hand

$$
\begin{aligned}
\left\|\llbracket u \rrbracket_{h}-\llbracket u \rrbracket_{h}^{u_{p, o}}\right\|_{L^{2}\left(\Omega_{T}, \mathbf{R}^{N}\right)}^{2} & =\iint_{\Omega_{T}} \mathrm{e}^{-\frac{2 t}{h}}\left|u_{p, o}-u_{p, o, h}\right|^{2} \mathrm{~d} x \mathrm{~d} t \\
& \leq T\left\|u_{p, o}-u_{p, o, h}\right\|_{L^{2}\left(\Omega, \mathbf{R}^{N}\right)}^{2} .
\end{aligned}
$$

Therefore, we conclude that

$$
\lim _{h \downarrow 0} \sup _{\tau \in[0, T]}\left\|\left(\llbracket u \rrbracket_{h}-u\right)(\tau)\right\|_{L^{2}\left(\Omega, \mathbf{R}^{N}\right)}^{2} \leq 0,
$$

which means that

$$
\lim _{h \downarrow 0}\left\|\llbracket u \rrbracket_{h}-u\right\|_{L^{\infty}\left(0, T ; L^{2}\left(\Omega, \mathbf{R}^{N}\right)\right)}=0 .
$$

Observe that the assertions $\llbracket u \rrbracket_{h}(0) \in L^{2}\left(\Omega, \mathbf{R}^{N}\right)$ and $\partial_{t} \llbracket u \rrbracket_{h} \in L^{1}\left(0, T ; L^{2}\left(\Omega, \mathbf{R}^{N}\right)\right)$ together imply that $\llbracket u \rrbracket_{h} \in C^{0}\left([0, T] ; L^{2}\left(\Omega, \mathbf{R}^{N}\right)\right)$ for any $h>0$. Thus, we have shown that $u$ is the limit of functions in $C^{0}\left([0, T] ; L^{2}\left(\Omega, \mathbf{R}^{N}\right)\right)$ with respect to the norm in $L^{\infty}\left(0, T ; L^{2}\left(\Omega, \mathbf{R}^{N}\right)\right)$. Consequently, we have that $u \in C^{0}\left([0, T] ; L^{2}\left(\Omega, \mathbf{R}^{N}\right)\right)$.

In order to prove a corresponding result for the case $p=1$, we need the following technical lemma.

Lemma A.2. Assume that $u \in L_{w *}^{1}\left(0, T ; \operatorname{BV}\left(\mathbf{R}^{n}, \mathbf{R}^{N}\right)\right), u_{o} \in L^{2}\left(\mathbf{R}^{n}, \mathbf{R}^{N}\right)$ and that $\llbracket u \rrbracket_{h}$ denotes the time mollification according to $(2.2)$ with initial datum $u_{o, h}:=$ $u_{o} * \phi_{\varepsilon(h)}$, where $\varepsilon(h):=h^{\frac{1}{2(n+1)}}$ and $\phi_{\varepsilon(h)}$ denotes the standard mollifier in $\mathbf{R}^{n}$. Then, there exists a (not relabelled) subsequence such that

$$
\left|\left(\mathcal{L}^{n}, D \llbracket u \rrbracket_{h}(t)\right)\right|(\bar{\Omega}) \rightarrow\left|\left(\mathcal{L}^{n}, D u(t)\right)\right|(\bar{\Omega})
$$

in the limit $h \downarrow 0$ holds true for a.e. $t \in[0, T]$. 
Proof. First, note that by Lemma 2.9 we have that $\llbracket u \rrbracket_{h} \in L_{w *}^{1}\left(0, T ; \operatorname{BV}\left(\mathbf{R}^{n}, \mathbf{R}^{N}\right)\right)$. Next, observe that $\left|\left(\mathcal{L}^{n}, D \llbracket u \rrbracket_{h}(t)\right)\right|(\bar{\Omega})=\left|D_{\left(x_{0}, x\right)}\left(x_{0}, \llbracket u \rrbracket_{h}(t)\right)\right|(\bar{\Omega})$, since the additional $n+N$ entries in $D_{\left(x_{0}, x\right)}\left(x_{0}, \llbracket u \rrbracket_{h}\right) \in \mathbf{R}^{(N+1) \times(n+1)}$ are zeroes. A straightforward calculation shows that $x_{0}=\llbracket x_{0} \rrbracket_{h}$ with initial values $x_{0}$. Hence, $D_{\left(x_{0}, x\right)}\left(x_{0}, \llbracket u \rrbracket_{h}(t)\right)=$ $D_{\left(x_{0}, x\right)} \llbracket\left(x_{0}, u\right) \rrbracket_{h}(t)$, where the time mollification on the right-hand side is defined with initial values $\left(x_{0}, u_{o, h}\right)$. Therefore, we need to prove that

$$
\lim _{h \downarrow 0}\left|D_{\left(x_{0}, x\right)} \llbracket\left(x_{0}, u\right) \rrbracket_{h}(t)\right|(\bar{\Omega})=\left|D_{\left(x_{0}, x\right)}\left(x_{0}, u(t)\right)\right|(\bar{\Omega})=\left|\left(\mathcal{L}^{n}, D u(t)\right)\right|(\bar{\Omega}) .
$$

To this end, note that $\llbracket\left(x_{0}, u\right) \rrbracket_{h}(t) \rightarrow\left(x_{0}, u(t)\right)$ in $L^{1}\left(\Omega, \mathbf{R}^{N+1}\right)$ for a.e. $t \in[0, T]$ by Lemma 2.7 and since $u_{o, h} \rightarrow u_{o}$ in $L^{2}\left(\Omega, \mathbf{R}^{N}\right)$. Therefore, by Theorem 2.3 applied to the total variation, we find that

$$
\left|D_{\left(x_{0}, x\right)}\left(x_{0}, u(t)\right)\right|(\bar{\Omega}) \leq \liminf _{h \rightarrow \infty}\left|D_{\left(x_{0}, x\right)} \llbracket\left(x_{0}, u\right) \rrbracket_{h}(t)\right|(\bar{\Omega}) .
$$

Further, let $\delta>0$ and assume that $\Omega^{\delta}:=\left\{x \in \mathbf{R}^{n}: \operatorname{dist}(x, \Omega)<\delta\right\}$ denotes the outer parallel set of $\Omega$ at distance $\delta$. Since $\Omega^{\delta}$ is open, we infer from Lemma 2.9 that

$$
\begin{aligned}
& \left|D_{\left(x_{0}, x\right)} \llbracket\left(x_{0}, u\right) \rrbracket_{h}(t)\right|(\bar{\Omega})=\lim _{\delta \downarrow 0}\left|D_{\left(x_{0}, x\right)} \llbracket\left(x_{0}, u\right) \rrbracket_{h}(t)\right|\left(\Omega^{\delta}\right) \\
& \leq \lim _{\delta \downarrow 0} \llbracket\left|D_{\left(x_{0}, x\right)}\left(x_{0}, u\right)\right|\left(\Omega^{\delta}\right) \rrbracket_{h}(t)=\llbracket\left|D_{\left(x_{0}, x\right)}\left(x_{0}, u\right)\right|(\bar{\Omega}) \rrbracket_{h}(t)
\end{aligned}
$$

Here, the mollification on the right-hand side is defined according to (2.2) with initial values $\left|D_{\left(x_{0}, x\right)}\left(x_{0}, u_{o, h}\right)\right|(\bar{\Omega})$. We claim that there exists a (not relabelled) subsequence such that

$$
\llbracket\left|D_{\left(x_{0}, x\right)}\left(x_{0}, u\right)\right|(\bar{\Omega}) \rrbracket_{h} \rightarrow\left|D_{\left(x_{0}, x\right)}\left(x_{0}, u\right)\right|(\bar{\Omega})
$$

in $L^{1}(0, T)$ as $h \downarrow 0$. Indeed, applying Lemma 2.7 with $X=\mathbf{R}$ to the time mollification with zero initial datum, we find that $\llbracket\left|D_{\left(x_{0}, x\right)}\left(x_{0}, u\right)\right|(\bar{\Omega}) \rrbracket_{h}^{o} \rightarrow\left|D_{\left(x_{0}, x\right)}\left(x_{0}, u\right)\right|(\bar{\Omega})$ in $L^{1}(0, T)$ as $h \downarrow 0$. Furthermore, by means of Lemma 2.12, we estimate

$$
\begin{aligned}
& \left\|\llbracket\left|D_{\left(x_{0}, x\right)}\left(x_{0}, u\right)\right|(\bar{\Omega}) \rrbracket_{h}^{o}-\llbracket\left|D_{\left(x_{0}, x\right)}\left(x_{0}, u\right)\right|(\bar{\Omega}) \rrbracket_{h}\right\|_{L^{1}(0, T)} \\
& =\int_{0}^{T} e^{-\frac{t}{h}}\left|D_{\left(x_{0}, x\right)}\left(x_{0}, u_{o, h}\right)\right|(\bar{\Omega}) \mathrm{d} t \leq h \int_{\Omega} \sqrt{1+\left|D u_{o, h}\right|^{2}} \mathrm{~d} x \\
& \leq h\left(|\Omega|+c(n,|\Omega|) \varepsilon(h)^{-n-1}\left\|u_{o}\right\|_{L^{2}\left(\Omega, \mathbf{R}^{N}\right)}\right) \\
& =h|\Omega|+c(n,|\Omega|) \sqrt{h}\left\|u_{o}\right\|_{L^{2}\left(\Omega, \mathbf{R}^{N}\right)} .
\end{aligned}
$$

Combining the preceding two observations yields the asserted convergence. Passing to a subsequence, we may assume that $\llbracket\left|D_{\left(x_{0}, x\right)}\left(x_{0}, u\right)\right|(\bar{\Omega}) \rrbracket_{h}(t) \rightarrow\left|D_{\left(x_{0}, x\right)}\left(x_{0}, u(t)\right)\right|(\bar{\Omega})$ for a.e. $t \in[0, T]$ in the limit $h \downarrow 0$. Using this fact in the right-hand side of (A.2) and combining the resulting estimate with (A.1) yields the claim of the lemma.

Lemma A.3. Assume that $u_{o} \in L^{2}\left(\Omega, \mathbf{R}^{N}\right)$ and that $u \in L^{\infty}\left(0, T ; L^{2}\left(\Omega, \mathbf{R}^{N}\right)\right) \cap$ $L_{w *}^{1}\left(0, T ; \operatorname{BV}\left(\Omega, \mathbf{R}^{N}\right)\right)$ is a variational solution to $(1.3)$ with initial datum $u_{o}$ in the sense of Definition 1.1. Then, $u \in C^{0}\left([0, T] ; L^{2}\left(\Omega, \mathbf{R}^{N}\right)\right)$.

Proof. Let $h>0$ and $\varepsilon=\varepsilon(h):=h^{\frac{1}{2(n+1)}}$. By means of Lemma 2.5 we extend $u$ to $\mathbf{R}^{n} \times(0, T)$. We consider the mollification $\llbracket u \rrbracket_{h}$ defined according to $(2.2)$ with initial values $u_{o, h}:=u_{o} * \phi_{\varepsilon(h)}$, where $u_{o}$ is extended to $\mathbf{R}^{n}$ by zero and $\phi_{\varepsilon}$ denotes the standard mollifier in $\mathbf{R}^{n}$. By Lemma 2.9 we have that $\llbracket u \rrbracket_{h} \in L_{w *}^{1}\left(0, T ; \mathrm{BV}\left(\Omega, \mathbf{R}^{N}\right)\right)$ with 
initial values $\llbracket u \rrbracket_{h}(0)=u_{o, h} \in L^{2}\left(\Omega, \mathbf{R}^{N}\right)$. Moreover, $\partial_{t} \llbracket u \rrbracket_{h} \in L^{1}\left(0, T ; L^{2}\left(\Omega, \mathbf{R}^{N}\right)\right)$ by (2.3). Noting that by (2.3) we have

$$
\iint_{\Omega_{\tau}} \partial_{t} \llbracket u \rrbracket_{h} \cdot\left(\llbracket u \rrbracket_{h}-u\right) \mathrm{d} x \mathrm{~d} t=-\frac{1}{h} \iint_{\Omega_{\tau}}\left|\llbracket u \rrbracket_{h}-u\right|^{2} \mathrm{~d} x \mathrm{~d} t \leq 0,
$$

by (1.13) with the choice $\llbracket u \rrbracket_{h}$ as comparison map, we find that

$$
\begin{aligned}
& \frac{1}{2}\left\|\left(\llbracket u \rrbracket_{h}-u\right)(\tau)\right\|_{L^{2}\left(\Omega, \mathbf{R}^{N}\right)}^{2} \\
& \leq \int_{0}^{T} \mathcal{F}\left[\llbracket u \rrbracket_{h}\right]-\mathcal{F}[u] \mathrm{d} t+\iint_{\Omega_{\tau}} g\left(x, \llbracket u \rrbracket_{h}\right)-g(x, u) \mathrm{d} x \mathrm{~d} t+\frac{1}{2}\left\|u_{o, h}-u_{o}\right\|_{L^{2}\left(\Omega, \mathbf{R}^{N}\right)}^{2}
\end{aligned}
$$

for a.e. $\tau \in[0, T]$. Observe that the last two terms on the right-hand side of the preceding inequality can be treated in exactly the same way as the corresponding terms in the proof of Lemma A.1. It remains to show that the first term on the right-hand side of the preceding inequality vanishes in the limit $h \downarrow 0$ (for a suitable subsequence). By Lemma A.2 there exists a (not relabelled) subsequence such that for a.e. $t \in[0, T]$

$$
\lim _{h \downarrow 0}\left|\left(\mathcal{L}^{n}, D \llbracket u \rrbracket_{h}(t)\right)\right|(\bar{\Omega})=\left|\left(\mathcal{L}^{n}, D u(t)\right)\right|(\bar{\Omega}) .
$$

Hence, by Theorem 2.4 we find that $\mathcal{F}\left[\llbracket u \rrbracket_{h}(t)\right] \rightarrow \mathcal{F}[u(t)]$ as $h \downarrow 0$ for a.e. $t \in[0, T]$. Moreover, by the growth conditions (1.1) and (1.7) and Lemma 2.9 we have that

$$
\begin{aligned}
\mathcal{F}\left[\llbracket u \rrbracket_{h}(t)\right] & \leq \int_{\Omega} L\left(1+\left|\nabla \llbracket u \rrbracket_{h}(t)\right|\right) \mathrm{d} x+\int_{\Omega} L \mathrm{~d}\left|D^{s} \llbracket u \rrbracket_{h}(t)\right| \\
& =L\left(|\Omega|+\left|D \llbracket u \rrbracket_{h}(t)\right|\right)(\Omega) \\
& \leq L\left(|\Omega|+\llbracket|D u|(\Omega) \rrbracket_{h}(t)\right),
\end{aligned}
$$

where the mollification on the right-hand side of the preceding inequality is defined according to $(2.2)$ with initial datum $\left|D u_{o, h}\right|(\Omega)$. Since by Lemma 2.7 we know for the time mollification with zero initial values that $\llbracket|D u|(\Omega) \rrbracket_{h}^{o} \rightarrow|D u|(\Omega)$ in $L^{1}(0, T)$ as $h \downarrow 0$ and by Lemma 2.12 we obtain that

$$
\begin{aligned}
\left\|\llbracket|D u|(\Omega) \rrbracket_{h}-\llbracket|D u|(\Omega) \rrbracket_{h}^{o}\right\|_{L^{1}(0, T)} & =\int_{0}^{T} e^{-\frac{t}{h}} \mathrm{~d} t \int_{\Omega}\left|D u_{o, h}\right| \mathrm{d} x \\
& \leq h c(n,|\Omega|) \varepsilon(h)^{-n-1}\left\|u_{o}\right\|_{L^{2}\left(\Omega, \mathbf{R}^{N}\right)} \\
& =c(n,|\Omega|) \sqrt{h}\left\|u_{o}\right\|_{L^{2}\left(\Omega, \mathbf{R}^{N}\right)},
\end{aligned}
$$

we conclude that $\llbracket|D u|(\Omega) \rrbracket_{h} \rightarrow|D u|(\Omega)$ in $L^{1}(0, T)$ in the limit $h \downarrow 0$. Recalling (A.3) and using this fact, by a version of the dominated convergence theorem we deduce that

$$
\lim _{h \downarrow 0} \int_{0}^{\tau} \mathcal{F}\left[\llbracket u \rrbracket_{h}\right] \mathrm{d} t=\int_{0}^{\tau} \mathcal{F}[u] \mathrm{d} t
$$

for any $\tau \in[0, T]$. This yields the claimed convergence. Therefore, taking the supremum over $\tau \in[0, T]$, we infer that

$$
\lim _{h \downarrow 0}\left\|\llbracket u \rrbracket_{h}-u\right\|_{L^{\infty}\left(0, T ; L^{2}\left(\Omega, \mathbf{R}^{N}\right)\right)}=0 .
$$

This means that $u$ is the limit of maps $\llbracket u \rrbracket_{h} \in C^{0}\left([0, T] ; L^{2}\left(\Omega, \mathbf{R}^{N}\right)\right)$ with respect to the norm in $L^{\infty}\left(0, T ; L^{2}\left(\Omega, \mathbf{R}^{N}\right)\right)$. Therefore, we find that $u \in C^{0}\left([0, T] ; L^{2}\left(\Omega, \mathbf{R}^{N}\right)\right)$.

The proof of the following lemma is similar to the proof of [15, Lemma 3.3]. 
Lemma A.4. There is at most one variational solution to (1.4) with initial values $u_{p, o} \in L^{2}\left(\Omega, \mathbf{R}^{N}\right)$ in the sense of Definition 1.3.

Proof. Let $h>0$ and $\varepsilon(h):=h^{\frac{1}{2(n+1) p}}$. Assume that $u_{1}, u_{2} \in C^{0}\left([0, T] ; L^{2}(\Omega\right.$, $\left.\left.\mathbf{R}^{N}\right)\right) \cap L^{p}\left(0, T ; W^{1, p}\left(\Omega, \mathbf{R}^{N}\right)\right)$ are both variational solutions in the sense of Definition 1.3. For $v:=\frac{1}{2}\left(u_{1}+u_{2}\right)$ we consider mollifications $\llbracket v \rrbracket_{h}$ according to $(2.2)$ with initial datum $u_{p, o, h}:=u_{p, o} * \phi_{\varepsilon(h)}$, where $u_{p, o}$ is extended to $\mathbf{R}^{n}$ outside of $\Omega$ by zero and $\phi_{\varepsilon(h)}$ denotes the standard mollifier in $\mathbf{R}^{n}$. Observe that these mollifications are admissible comparison maps in the variational inequality (1.17) for $u_{1}$ and $u_{2}$, since $\llbracket v \rrbracket_{h}(0)=u_{p, o, h} \in L^{2}\left(\Omega, \mathbf{R}^{N}\right)$ and $\llbracket v \rrbracket_{h} \in L^{p}\left(0, T ; W^{1, p}\left(\Omega, \mathbf{R}^{N}\right)\right)$ by Lemma 2.7 and $\partial_{t} \llbracket v \rrbracket_{h} \in L^{1}\left(0, T ; L^{2}\left(\Omega, \mathbf{R}^{N}\right)\right)$ by $(2.3)$. Adding both inequalities and recalling that $u_{1}+u_{2}=2 v$, we obtain that

$$
\begin{aligned}
& \frac{1}{2}\left\|\left(\llbracket v \rrbracket_{h}-u_{1}\right)(\tau)\right\|_{L^{2}\left(\Omega, \mathbf{R}^{N}\right)}^{2}+\frac{1}{2}\left\|\left(\llbracket v \rrbracket_{h}-u_{2}\right)(\tau)\right\|_{L^{2}\left(\Omega, \mathbf{R}^{N}\right)}^{2} \\
& \leq \iint_{\Omega_{\tau}} 2 f^{p}\left(x, D \llbracket v \rrbracket_{h}\right)-f^{p}\left(x, D u_{1}\right)-f^{p}\left(x, D u_{2}\right) \mathrm{d} x \mathrm{~d} t \\
& \quad+\iint_{\Omega_{\tau}} 2 g\left(x, \llbracket v \rrbracket_{h}\right)-g\left(x, u_{1}\right)-g\left(x, u_{2}\right) \mathrm{d} x \mathrm{~d} t \\
& \quad+2 \iint_{\Omega_{\tau}} \partial_{t} \llbracket v \rrbracket_{h} \cdot\left(\llbracket v \rrbracket_{h}-v\right) \mathrm{d} x \mathrm{~d} t+\left\|u_{p, o, h}-u_{p, o}\right\|_{L^{2}\left(\Omega, \mathbf{R}^{N}\right)}^{2}
\end{aligned}
$$

for a.e. $\tau \in[0, T]$. First note that $u_{p, o, h} \rightarrow u_{p, o}$ in $L^{2}\left(\Omega, \mathbf{R}^{N}\right)$ as $h \downarrow 0$. By Lemma 2.13 we have that $D \llbracket v \rrbracket_{h} \rightarrow D v$ in $L^{p}\left(\Omega_{T}, \mathbf{R}^{N n}\right)$ as $h \downarrow 0$. Further, applying Lemma 2.10 to $f^{p}$ with $m=N n$ and $p_{o}=p$ and using the convexity condition (1.15), we conclude that

$$
\begin{aligned}
\lim _{h \downarrow 0} \iint_{\Omega_{\tau}} 2 f^{p}\left(x, D \llbracket v \rrbracket_{h}\right) \mathrm{d} x \mathrm{~d} t & =\iint_{\Omega_{\tau}} 2 f^{p}\left(x, \frac{1}{2} D u_{1}+\frac{1}{2} D u_{2}\right) \mathrm{d} x \mathrm{~d} t \\
& \leq \iint_{\Omega_{\tau}} f^{p}\left(x, D u_{1}\right)+f^{p}\left(x, D u_{2}\right) \mathrm{d} x \mathrm{~d} t .
\end{aligned}
$$

Similarly, we infer from the fact that $\llbracket v \rrbracket_{h} \rightarrow v$ in $L^{2}\left(\Omega_{T}, \mathbf{R}^{N}\right)$, cf. the proof of Lemma A.1, from Lemma 2.10 with $m=N, p_{o}=p=2$ and from (1.11) that

$$
\begin{aligned}
\lim _{h \downarrow 0} \iint_{\Omega_{\tau}} 2 g\left(x, \llbracket v \rrbracket_{h}\right) \mathrm{d} x \mathrm{~d} t & =\iint_{\Omega_{\tau}} 2 g\left(x, \frac{1}{2} u_{1}+\frac{1}{2} u_{2}\right) \mathrm{d} x \mathrm{~d} t \\
& \leq \iint_{\Omega_{\tau}} g\left(x, u_{1}\right)+g\left(x, u_{2}\right) \mathrm{d} x \mathrm{~d} t .
\end{aligned}
$$

Next, by (2.3) we obtain that

$$
2 \iint_{\Omega_{\tau}} \partial_{t} \llbracket v \rrbracket_{h} \cdot\left(\llbracket v \rrbracket_{h}-v\right) \mathrm{d} x \mathrm{~d} t=-\frac{2}{h} \iint_{\Omega_{\tau}}\left|\llbracket v \rrbracket_{h}-v\right|^{2} \mathrm{~d} x \mathrm{~d} t \leq 0 .
$$

Finally, we deduce that $\llbracket v \rrbracket_{h} \rightarrow v$ in $L^{\infty}\left(0, T ; L^{2}\left(\Omega, \mathbf{R}^{N}\right)\right)$. To this end note that we have for the time mollification $\llbracket v \rrbracket_{h}^{u_{p, o}}=\mathrm{e}^{-\frac{t}{h}}\left(u_{p, o}-u_{p, o, h}\right)+\llbracket v \rrbracket_{h}$ with initial datum $u_{p, o}$ that $\llbracket v \rrbracket_{h}^{u_{p, o}} \rightarrow v$ in $L^{\infty}\left(0, T ; L^{2}\left(\Omega, \mathbf{R}^{N}\right)\right)$ by Lemma 2.7 . Thus, it remains to compute

$$
\sup _{t \in[0, T]}\left\|\mathrm{e}^{-\frac{t}{h}}\left(u_{p, o}-u_{p, o, h}\right)\right\|_{L^{2}\left(\Omega, \mathbf{R}^{N}\right)}=\left\|u_{p, o}-u_{p, o, h}\right\|_{L^{2}\left(\Omega, \mathbf{R}^{N}\right)} \rightarrow 0
$$


as $h \downarrow 0$, which yields the claim. Hence, we conclude that for $j \in\{1,2\}$

$$
\lim _{h \downarrow 0}\left\|\left(\llbracket v \rrbracket_{h}-u_{j}\right)(\tau)\right\|_{L^{2}\left(\Omega, \mathbf{R}^{N}\right)}^{2}=\frac{1}{4}\left\|\left(u_{1}-u_{2}\right)(\tau)\right\|_{L^{2}\left(\Omega, \mathbf{R}^{N}\right)}^{2} .
$$

Combining the preceding computations and passing to the limit $h \downarrow 0$ in (A.4), we infer that

$$
\frac{1}{4}\left\|\left(u_{1}-u_{2}\right)(\tau)\right\|_{L^{2}\left(\Omega, \mathbf{R}^{N}\right)}^{2} \leq 0
$$

for a.e. $\tau \in[0, T]$, which implies that $u_{1}=u_{2}$ a.e. in $\Omega_{T}$.

A corresponding uniqueness result holds true in the case $p=1$.

Lemma A.5. There is at most one variational solution to (1.3) with initial values $u_{o} \in L^{2}\left(\Omega, \mathbf{R}^{N}\right)$ in the sense of Definition 1.1.

Proof. Let $h>0$ and $\varepsilon(h):=h^{\frac{1}{2(n+1)}}$. Assume that $u_{1}, u_{2} \in L^{\infty}\left(0, T ; L^{2}\left(\Omega, \mathbf{R}^{N}\right)\right) \cap$ $L_{w *}^{1}\left(0, T ; \mathrm{BV}\left(\Omega, \mathbf{R}^{N}\right)\right)$ are both variational solutions in the sense of Definition 1.3. By Lemma A.3 we conclude that $u_{1}, u_{2} \in C^{0}\left([0, T] ; L^{2}\left(\Omega, \mathbf{R}^{N}\right)\right)$. Further, by Lemma 2.5 we extend $u_{1}, u_{2}$ to $\mathbf{R}^{n} \times(0, T)$. For $v:=\frac{1}{2}\left(u_{1}+u_{2}\right)$ we consider mollifications $\llbracket v \rrbracket_{h}$ according to (2.2) with initial datum $u_{o, h}:=u_{o} * \phi_{\varepsilon(h)}$, where $u_{o}$ is extended to $\mathbf{R}^{n}$ outside of $\Omega$ by zero and $\phi_{\varepsilon(h)}$ denotes the standard mollifier in $\mathbf{R}^{n}$. Observe that these mollifications are admissible comparison maps in the variational inequality (1.13) for $u_{1}$ and $u_{2}$, since $\llbracket v \rrbracket_{h}(0)=u_{o, h} \in L^{2}\left(\Omega, \mathbf{R}^{N}\right)$ and $\llbracket v \rrbracket_{h} \in L_{w *}^{1}\left(0, T ; \mathrm{BV}\left(\Omega, \mathbf{R}^{N}\right)\right)$ by Lemma 2.9 and $\partial_{t} \llbracket v \rrbracket_{h} \in L^{1}\left(0, T ; L^{2}\left(\Omega, \mathbf{R}^{N}\right)\right)$ by (2.3). Adding both inequalities, using (2.3) to conclude that the term involving the time derivative is non-positive and recalling that $u_{1}+u_{2}=2 v$, we obtain that

$$
\begin{aligned}
& \frac{1}{2}\left\|\left(\llbracket v \rrbracket_{h}-u_{1}\right)(\tau)\right\|_{L^{2}\left(\Omega, \mathbf{R}^{N}\right)}^{2}+\frac{1}{2}\left\|\left(\llbracket v \rrbracket_{h}-u_{2}\right)(\tau)\right\|_{L^{2}\left(\Omega, \mathbf{R}^{N}\right)}^{2} \\
& \leq \int_{0}^{\tau} 2 \mathcal{F}\left[\llbracket v \rrbracket_{h}\right]-\mathcal{F}\left[u_{1}\right]-\mathcal{F}\left[u_{2}\right] \mathrm{d} t \\
& \quad+\iint_{\Omega_{\tau}} 2 g\left(x, \llbracket v \rrbracket_{h}\right)-g\left(x, u_{1}\right)-g\left(x, u_{2}\right) \mathrm{d} x \mathrm{~d} t+\left\|u_{o, h}-u_{o}\right\|_{L^{2}\left(\Omega, \mathbf{R}^{N}\right)}^{2}
\end{aligned}
$$

for a.e. $\tau \in[0, T]$. The last two terms on the right-hand side of (A.5) can be treated exactly as the corresponding terms in the proof of Lemma A.4. Thus, it remains to show that the limit of the first term appearing on the right-hand side of (A.5) is non-positive. By Lemma A.2 we obtain that

$$
\lim _{h \downarrow 0}\left|\left(\mathcal{L}^{n}, D \llbracket v \rrbracket_{h}(t)\right)\right|(\bar{\Omega})=\left|\left(\mathcal{L}^{n}, D v(t)\right)\right|(\bar{\Omega})
$$

for a.e. $t \in[0, T]$ and a (not relabelled) subsequence. Hence, by Theorem 2.4 we find that

$$
\lim _{h \downarrow 0} \mathcal{F}\left[\llbracket v \rrbracket_{h}(t)\right]=\mathcal{F}[v(t)]
$$

for a.e. $t \in[0, T]$. Moreover, by $(1.1),(1.7)$ and Lemma 2.9 we conclude that

$$
\begin{aligned}
\mathcal{F}\left[\llbracket v \rrbracket_{h}(t)\right] & \leq \int_{\Omega} L\left(1+\left|\nabla \llbracket v \rrbracket_{h}(t)\right|\right) \mathrm{d} x+\int_{\Omega} L \mathrm{~d}\left|D^{s} \llbracket v \rrbracket_{h}(t)\right| \\
& =L\left(|\Omega|+\left|D \llbracket v \rrbracket_{h}(t)\right|\right)(\Omega) \\
& \leq L\left(|\Omega|+\llbracket|D v|(\Omega) \rrbracket_{h}(t)\right),
\end{aligned}
$$

where the mollification on the right-hand side of the preceding inequality is defined according to $(2.2)$ with initial datum $\left|D u_{o, h}\right|(\Omega)$. Since by Lemma 2.7 we know for 
the time mollification with zero initial values that $\llbracket|D v|(\Omega) \rrbracket_{h}^{o} \rightarrow|D v|(\Omega)$ in $L^{1}(0, T)$ as $h \downarrow 0$ and by Lemma 2.12 we obtain that

$$
\begin{aligned}
\left\|\llbracket|D v|(\Omega) \rrbracket_{h}-\llbracket|D v|(\Omega) \rrbracket_{h}^{o}\right\|_{L^{1}(0, T)} & =\int_{0}^{T} e^{-\frac{t}{h}} \mathrm{~d} t \int_{\Omega}\left|D u_{o, h}\right| \mathrm{d} x \\
& \leq h c(n,|\Omega|) \varepsilon(h)^{-n-1}\left\|u_{o}\right\|_{L^{2}\left(\Omega, \mathbf{R}^{N}\right)} \\
& =c(n,|\Omega|) \sqrt{h}\left\|u_{o}\right\|_{L^{2}\left(\Omega, \mathbf{R}^{N}\right)} .
\end{aligned}
$$

Thus, we conclude that $\llbracket|D v|(\Omega) \rrbracket_{h} \rightarrow|D v|(\Omega)$ in $L^{1}(0, T)$ in the limit $h \downarrow 0$. Hence, using (A.6), a version of the dominated convergence theorem and the convexity of $\mathcal{F}$, we deduce that

$$
\begin{aligned}
& \lim _{h \downarrow 0} \int_{0}^{\tau} 2 \mathcal{F}\left[\llbracket v \rrbracket_{h}\right]-\mathcal{F}\left[u_{1}\right]-\mathcal{F}\left[u_{2}\right] \mathrm{d} t \\
& =\int_{0}^{\tau} 2 \mathcal{F}\left[\frac{1}{2} u_{1}+\frac{1}{2} u_{2}\right]-\mathcal{F}\left[u_{1}\right]-\mathcal{F}\left[u_{2}\right] \mathrm{d} t \leq 0
\end{aligned}
$$

for any $\tau \in[0, T]$. Inserting this into (A.5), we infer that

$$
0 \geq \lim _{h \downarrow 0}\left\|\left(\llbracket v \rrbracket_{h}-u_{j}\right)(\tau)\right\|_{L^{2}\left(\Omega, \mathbf{R}^{N}\right)}^{2}=\frac{1}{4}\left\|\left(u_{1}-u_{2}\right)(\tau)\right\|_{L^{2}\left(\Omega, \mathbf{R}^{N}\right)}^{2}
$$

holds true for a.e. $\tau \in[0, T]$. Hence, $u_{1}=u_{2}$ a.e. in $\Omega_{T}$.

\section{Appendix B. Localization}

Here, we establish that any map $u \in C^{0}\left([0, T] ; L^{2}\left(\Omega, \mathbf{R}^{N}\right)\right) \cap L^{p}\left(0, T ; W^{1, p}\left(\Omega, \mathbf{R}^{N}\right)\right)$ satisfying the variational inequality $(1.17)$ on the whole time interval $[0, T]$ is a variational solution in the sense of Definition 1.3 for any sub-cylinder $\Omega_{\tau} \subset \Omega_{T}$. For the proof strategy of. [15, Sec. 3.3.1] The precise statement is as follows.

Lemma B.1. Assume that $u_{p, o}$ is given as in (1.16), $f^{p}$ fulfills (1.14) and (1.15) and $g$ fulfills (1.10) and (1.11). Further, suppose that

$$
\begin{aligned}
& \iint_{\Omega_{T}}\left[f^{p}(x, D u)+g(x, u)\right] \mathrm{d} x \mathrm{~d} t \\
& \leq \iint_{\Omega_{T}} \partial_{t} v \cdot(v-u) \mathrm{d} x \mathrm{~d} t+\iint_{\Omega_{T}}\left[f^{p}(x, D v)+g(x, v)\right] \mathrm{d} x \mathrm{~d} t \\
& \quad-\frac{1}{2}\|(v-u)(T)\|_{L^{2}\left(\Omega, \mathbf{R}^{N}\right)}^{2}+\frac{1}{2}\left\|v(0)-u_{p, o}\right\|_{L^{2}\left(\Omega, \mathbf{R}^{N}\right)}^{2}
\end{aligned}
$$

holds true for any map $v \in L^{p}\left(0, T ; W^{1, p}\left(\Omega, \mathbf{R}^{N}\right)\right)$ with $\partial_{t} v \in L^{1}\left(0, T ; L^{2}\left(\Omega, \mathbf{R}^{N}\right)\right)$ and $v(0) \in L^{2}\left(\Omega, \mathbf{R}^{N}\right)$. Then, $u$ is a variational solution in the sense of Definition 1.3 for any sub-cylinder $\Omega_{\tau} \subset \Omega_{T}$.

Proof. Let $h>0$ and $\varepsilon(h):=h^{\frac{1}{2(n+1) p}}$. In the following, $\llbracket u \rrbracket_{h}$ denotes the mollification according to $(2.2)$ with initial values $u_{p, o, h}:=u_{p, o} * \phi_{\varepsilon(h)}$, where $u_{p, o}$ is extended to $\mathbf{R}^{n}$ by zero and $\phi_{\varepsilon(h)}$ is the standard mollifier in $\mathbf{R}^{n}$. Note that $\llbracket u \rrbracket_{h}$ is an admissible comparison map in (B.1). Furthermore, we consider an arbitrary map $v \in L^{p}\left(0, \tau ; W^{1, p}\left(\Omega, \mathbf{R}^{N}\right)\right)$ with $\partial_{t} v \in L^{1}\left(0, \tau ; L^{2}\left(\Omega, \mathbf{R}^{N}\right)\right)$ and $v(0) \in L^{2}\left(\Omega, \mathbf{R}^{N}\right)$ and for $\theta \in(0, \tau)$ the cut-off function

$$
\xi_{\theta}(t):=\chi_{[0, \tau-\theta]}(t)+\frac{\tau-t}{\theta} \chi_{(\tau-\theta, \tau]}(t) .
$$


Since $\tilde{v}:=\xi_{\theta} v+\left(1-\xi_{\theta}\right) \llbracket u \rrbracket_{h}$ is an admissible comparison map in (B.1), we obtain that

$$
\begin{aligned}
& \iint_{\Omega_{T}}\left[f^{p}(x, D u)+g(x, u)\right] \mathrm{d} x \mathrm{~d} t \\
& \leq \iint_{\Omega_{T}} \partial_{t} \tilde{v} \cdot(\tilde{v}-u) \mathrm{d} x \mathrm{~d} t+\iint_{\Omega_{T}}\left[f^{p}(x, D \tilde{v})+g(x, \tilde{v})\right] \mathrm{d} x \mathrm{~d} t \\
& \quad-\frac{1}{2}\left\|\left(\llbracket u \rrbracket_{h}-u\right)(T)\right\|_{L^{2}\left(\Omega, \mathbf{R}^{N}\right)}^{2}+\frac{1}{2}\left\|v(0)-u_{p, o}\right\|_{L^{2}\left(\Omega, \mathbf{R}^{N}\right)}^{2} .
\end{aligned}
$$

In order to pass to the limit $\theta \downarrow 0$, we analyze the terms appearing on the right-hand side of the preceding inequality separately. We re-write the first term as

$$
\begin{aligned}
& \iint_{\Omega_{T}} \partial_{t} \tilde{v} \cdot(\tilde{v}-u) \mathrm{d} x \mathrm{~d} t \\
& =\iint_{\Omega \times(0, \tau-\theta)} \partial_{t} v \cdot(v-u) \mathrm{d} x \mathrm{~d} t+\iint_{\Omega \times(\tau, T)} \partial_{t} \llbracket u \rrbracket_{h} \cdot\left(\llbracket u \rrbracket_{h}-u\right) \mathrm{d} x \mathrm{~d} t \\
& \quad+\iint_{\Omega \times(\tau-\theta, \tau)} \xi_{\theta}^{\prime} \xi_{\theta}\left|v-\llbracket u \rrbracket_{h}\right|^{2} \mathrm{~d} x \mathrm{~d} t \\
& \quad+\iint_{\Omega \times(\tau-\theta, \tau)} \xi_{\theta}^{\prime}\left(\llbracket u \rrbracket_{h}-u\right) \cdot\left(v-\llbracket u \rrbracket_{h}\right) \mathrm{d} x \mathrm{~d} t \\
& \quad+\iint_{\Omega \times(\tau-\theta, \tau)}\left[\xi_{\theta} \partial_{t} v+\left(1-\xi_{\theta}\right) \partial_{t} \llbracket u \rrbracket_{h}\right]\left[\xi_{\theta}(v-u)+\left(1-\xi_{\theta}\right)\left(\llbracket u \rrbracket_{h}-u\right)\right] \mathrm{d} x \mathrm{~d} t \\
& =: \mathrm{I}_{\theta}+\mathrm{II}+\mathrm{III}_{\theta}+\mathrm{IV}_{\theta}+\mathrm{V}_{\theta},
\end{aligned}
$$

where the meaning of $\mathrm{I}_{\theta}, \mathrm{II}$ and $\mathrm{III}_{\theta}-\mathrm{V}_{\theta}$ is obvious in this context. Note that

$$
\lim _{\theta \downarrow 0} \mathrm{I}_{\theta}=\iint_{\Omega_{\tau}} \partial_{t} v \cdot(v-u) \mathrm{d} x \mathrm{~d} t .
$$

By (2.3) we have that

$$
\mathrm{II}=\iint_{\Omega \times(\tau, T)}-\frac{1}{h}\left|\llbracket u \rrbracket_{h}-u\right|^{2} \mathrm{~d} x \mathrm{~d} t \leq 0 .
$$

Since $u, \llbracket u \rrbracket_{h}, v \in C^{0}\left([0, \tau] ; L^{2}\left(\Omega, \mathbf{R}^{N}\right)\right)$, we conclude that

$$
\begin{aligned}
& \int_{(\tau-\theta, \tau)} \xi_{\theta}^{\prime} \xi_{\theta} \mathrm{d} t \min _{t \in(\tau-\theta, \tau)}\left\|\left(v-\llbracket u \rrbracket_{h}\right)(t)\right\|_{L^{2}\left(\Omega, \mathbf{R}^{N}\right)}^{2} \leq \mathrm{III}_{\theta} \\
& \leq \int_{(\tau-\theta, \tau)} \xi_{\theta}^{\prime} \xi_{\theta} \mathrm{d} t \max _{t \in(\tau-\theta, \tau)}\left\|\left(v-\llbracket u \rrbracket_{h}\right)(t)\right\|_{L^{2}\left(\Omega, \mathbf{R}^{N}\right)}^{2}
\end{aligned}
$$

and hence, that

$$
\lim _{\theta \downarrow 0} \operatorname{III}_{\theta}=-\frac{1}{2}\left\|\left(v-\llbracket u \rrbracket_{h}\right)(\tau)\right\|_{L^{2}\left(\Omega, \mathbf{R}^{N}\right)}^{2} .
$$

Similarly, we obtain that

$$
\limsup _{\theta \downarrow 0}\left|\operatorname{IV}_{\theta}\right| \leq\left\|\left(\llbracket u \rrbracket_{h}-u\right) \cdot\left(v-\llbracket u \rrbracket_{h}\right)(\tau)\right\|_{L^{1}\left(\Omega, \mathbf{R}^{N}\right)} .
$$

Moreover, since $0 \leq \xi_{\theta} \leq 1, \partial_{t} \llbracket u \rrbracket_{h} \in L^{1}\left(0, T ; L^{2}\left(\Omega, \mathbf{R}^{N}\right)\right)$ by $(2.3)$ and $u, \llbracket u \rrbracket_{h}, v \in$ $C^{0}\left([0, \tau] ; L^{2}\left(\Omega, \mathbf{R}^{N}\right)\right)$ we find that

$$
\lim _{\theta \downarrow 0} \mathrm{~V}_{\theta}=0
$$


Next, we re-write

$$
\begin{aligned}
\iint_{\Omega_{T}}\left[f^{p}(x, D \tilde{v})+g(x, \tilde{v})\right] \mathrm{d} x \mathrm{~d} t= & \iint_{\Omega \times(0, \tau-\theta)}\left[f^{p}(x, D v)+g(x, v)\right] \mathrm{d} x \mathrm{~d} t \\
& +\iint_{\Omega \times(\tau-\theta, \tau)}\left[f^{p}(x, D \tilde{v})+g(x, \tilde{v})\right] \mathrm{d} x \mathrm{~d} t \\
& +\iint_{\Omega \times(\tau, T)}\left[f^{p}\left(x, D \llbracket u \rrbracket_{h}\right)+g\left(x, \llbracket u \rrbracket_{h}\right)\right] \mathrm{d} x \mathrm{~d} t .
\end{aligned}
$$

By the growth conditions (1.14) and (1.10) and the fact that $\tilde{v} \in L^{p}\left(0, \tau ; W^{1, p}\left(\Omega, \mathbf{R}^{N}\right)\right) \cap$ $C^{0}\left([0, \tau] ; L^{2}\left(\Omega, \mathbf{R}^{N}\right)\right)$, we conclude that

$$
\begin{aligned}
\lim _{\theta \downarrow 0} \iint_{\Omega_{T}}\left[f^{p}(x, D \tilde{v})+g(x, \tilde{v})\right] \mathrm{d} x \mathrm{~d} t= & \iint_{\Omega \times(0, \tau)}\left[f^{p}(x, D v)+g(x, v)\right] \mathrm{d} x \mathrm{~d} t \\
& +\iint_{\Omega \times(\tau, T)}\left[f^{p}\left(x, D \llbracket u \rrbracket_{h}\right)+g\left(x, \llbracket u \rrbracket_{h}\right)\right] \mathrm{d} x \mathrm{~d} t .
\end{aligned}
$$

Combining the preceding computations and passing to the limit $\theta \downarrow 0$ leads to the inequality

$$
\begin{aligned}
& \iint_{\Omega_{\tau}}\left[f^{p}(x, D u)+g(x, u)\right] \mathrm{d} x \mathrm{~d} t \\
& \leq \quad \iint_{\Omega_{\tau}} \partial_{t} v \cdot(v-u) \mathrm{d} x \mathrm{~d} t+\iint_{\Omega_{\tau}}\left[f^{p}(x, D v)+g(x, v)\right] \mathrm{d} x \mathrm{~d} t \\
& \quad-\frac{1}{2}\left\|\left(v-\llbracket u \rrbracket_{h}\right)(\tau)\right\|_{L^{2}\left(\Omega, \mathbf{R}^{N}\right)}^{2}+\frac{1}{2}\left\|v(0)-u_{o}\right\|_{L^{2}(\Omega)}^{2} \\
& \quad-\frac{1}{2}\left\|\left(\llbracket u \rrbracket_{h}-u\right)(T)\right\|_{L^{2}\left(\Omega, \mathbf{R}^{N}\right)}^{2}+\left\|\left(\llbracket u \rrbracket_{h}-u\right) \cdot\left(v-\llbracket u \rrbracket_{h}\right)(\tau)\right\|_{L^{1}\left(\Omega, \mathbf{R}^{N}\right)} \\
& \quad+\iint_{\Omega \times(\tau, T)}\left[f^{p}\left(x, D \llbracket u \rrbracket_{h}\right)-f^{p}(x, D u)\right] \mathrm{d} x \mathrm{~d} t \\
& \quad+\iint_{\Omega \times(\tau, T)}\left[g\left(x, \llbracket u \rrbracket_{h}\right)-g(x, u)\right] \mathrm{d} x \mathrm{~d} t .
\end{aligned}
$$

The last four terms of the preceding inequality vanish in the limit $h \downarrow 0$. To this end recall that $\llbracket u \rrbracket_{h} \rightarrow u$ in $L^{\infty}\left(0, T ; L^{2}\left(\Omega, \mathbf{R}^{N}\right)\right)$ as $h \downarrow 0$; cf. the proof of Lemma A.4. By Lemma 2.13, we have that $D \llbracket u \rrbracket_{h} \rightarrow D u$ in $L^{p}\left(\Omega_{T}, \mathbf{R}^{N n}\right)$ as $h \downarrow 0$. Hence, applying Lemma 2.10 with the choices $m=N n, p_{o}=p$ for the term containing $f^{p}$ and $m=N, p_{o}=p=2$ for the term associated with $g$ implies the claim. Altogether, this shows that $u$ is a variational solution on $\Omega_{\tau}$.

\section{References}

[1] Ambrosio, L., N. Fusco, and D. Pallara: Functions of bounded variation and free discontinuity problems. - Oxford Math. Monogr., Oxford Univ. Press, New York, 2000.

[2] Andreu, F., C. Ballester, V. Caselles, and J. M. Mazón: The Dirichlet problem for the total variation flow. - J. Funct. Anal. 180:2, 2001, 347-403, 2001.

[3] Andreu, F., C. Ballester, V. Caselles, and J. M. Mazón: Minimizing total variation flow. - Differential Integral Equations 14:3, 2001, 321-360.

[4] Andreu, F., V. Caselles, J. I. Díaz, and J. M. Mazón: Some qualitative properties for the total variation flow. - J. Funct. Anal. 188:2, 2002, 516-547.

[5] Andreu, F., V. Caselles, and J. M. Mazón: Parabolic quasilinear equations minimizing linear growth functionals. - Progr. Math. 223, Birkhäuser Verlag, Basel, 2004. 
[6] Andreu, F., J. M. Mazón, and J. S. Moll: The total variation flow with nonlinear boundary conditions. - Asymptot. Anal. 43:1-2, 2005, 9-46.

[7] Andreu, F., J. M. Mazón, J. S. Moll, and V. Caselles: The minimizing total variation flow with measure initial conditions. - Commun. Contemp. Math. 6:3, 2004, 431-494.

[8] Andreu, F., V. Caselles, and J. M. Mazón: Existence and uniqueness of a solution for a parabolic quasilinear problem for linear growth functionals with $L^{1}$ data. - Math. Ann. 322, 2002, 139-206.

[9] Andreu, F., V. Caselles, and J. M. Mazón: The Cauchy problem for linear growth functionals. - J. Evol. Equ. 3, 2003, 39-65.

[10] Beck, L., and T. Schmidt: Convex duality and uniqueness for BV-minimizers. - J. Funct. Anal. 268:10, 2015, 3061-3107.

[11] Bellettini, G., V. Caselles, and M. Novaga: The total variation flow in $\mathbf{R}^{N}$. - J. Differential Equations 184:2, 2002, 475-525.

[12] Bögelein, V., F. DuzaAr, and P. Marcellini: Parabolic systems with $p, q$-growth: a variational approach. - Arch. Ration. Mech. Anal. 210:1, 2013, 219-267.

[13] Bögelein, V., F. Duzahr, and P. Marcellini A time dependent variational approach to image restoration. - SIAM J. Imaging Sci. 8:2, 2015, 968-1006.

[14] Bögelein, V., F. Duzaar, L. Schätzler, and C. Scheven: Existence for evolutionary problems with linear growth by stability methods. - J. Differential Equations, 2018, https://doi.org/10.1016/j.jde.2018.12.012.

[15] Bögelein, V., F. DuzaAR, and C. Scheven: The obstacle problem for parabolic minimizers. - J. Evol. Equ. 17:4, 2017, 1273-1310.

[16] Bögelein, V., F. DuzaAr, and C. Scheven: The obstacle problem for the total variation flow. - Ann. Sci. Éc. Norm. Supér. (4) 49:5, 2016, 1143-1188.

[17] Bögelein, V., F. DuzaAr, and C. Scheven: The total variation flow with time dependent boundary values. - Calc. Var. Partial Differential Equations 55:4, 2016, 108.

[18] DE León, S. S., and C. M. Webler: Global existence and uniqueness for the inhomogeneous 1-Laplace evolution equation. - NoDEA Nonlinear Differential Equations Appl. 22:5, 2015, $1213-1246$.

[19] Diestel, J., and J. Uhl: Vector measures. - Mathematical Surveys 15, Amer. Math. Soc., Providence, R.I., 1977.

[20] Fonseca, I., and S. Müller: Relaxation of quasiconvex functionals in BV $\left(\Omega, \boldsymbol{R}^{p}\right)$ for integrands $f(x, u, \nabla u)$. - Arch. Ration. Mech. Anal. 123, 1993, 1-49.

[21] Fujishima, Y., J. Habermann, J. Kinnunen, and J. Masson: Stability for parabolic quasiminimizers. - Potential Anal. 41:3, 2014, 983-1004.

[22] Gianazza, U., and C. Klaus: p-parabolic approximation of total variation flow solutions. Indiana Univ. Math. J. (to appear).

[23] Hardt, R., and X. Zhou: An evolution problem for linear growth functionals. - Comm. Partial Differential Equations 19:11\&12, 1994, 1879-1907.

[24] Kinnunen, J., and P. Lindqvist: Pointwise behaviour of semicontinuous supersolutions to a quasilinear parabolic equation. - Ann. Mat. Pura Appl. (4) 185:33, 2006, 411-435.

[25] Kinnunen, J., and M. Parviainen: Stability for degenerate parabolic equations. - Adv. Calc. Var. 3, 2010, 29-48.

[26] Kristensen, J., and F. Rindler: Characterization of generalized gradient Young measures generated by sequences in $W^{1,1}$ and BV. - Arch. Rational Mech. Anal. 197, 2010, 539-598.

[27] Kristensen, J., and F. Rindler: Relaxation of signed integral functionals in BV. - Calc. Var. Partial Differential Equations 37, 2010, 29-62. 
[28] Lichnewsky, A., and R. Temam: Pseudosolutions of the time-dependent minimal surface problem. - J. Differential Equations 30:3, 1978, 340-364.

[29] Marcellini, P.: Approximation of quasiconvex functions, and lower semicontinuity of multiple integrals. - Manuscripta Math. 51, 1985, 1-28.

[30] Rindler, F.: Lower semicontinuity and Young measures for integral functionals with linear growth. - Ph.D. Thesis, University of Oxford, 2011.

[31] Simon, J.: Compact sets in the space $L^{p}(0, T ; B)$. - Ann. Mat. Pura Appl. (IV) 146, 1987, 65-96.

[32] Spadaro, E., and U. Stefanelli: A variational view at the time-dependent minimal surface equation. - J. Evol. Equ. 11:4, 2011, 793-809.

[33] Tölle, J. M.: Convergence of solutions to the $p$-Laplace evolution equation as $p$ goes to $1 .-$ Preprint, arXiv:1103.0229, 2011.

Received 15 August 2018 • Accepted 8 February 2019 • Revised received 1 March 2019 